\title{
Development and Design Optimization of High fidelity Reduced Order Models for Dynamic Aeroelasticity Loads Analyses of Complex Airframes
}

\author{
by \\ Paul Vazhayil Thomas \\ B.Eng. in Aeronautical Engineering
}

A thesis submitted to the Faculty of Graduate and Postdoctoral Affairs in partial fulfillment of the requirements for the degree of

Master of Applied Science

in

Aerospace Engineering

Carleton University

Ottawa, Ontario

(C) 2018, Paul Vazhayil Thomas 


\section{Abstract}

Identification of aircraft critical loads envelope requires a lengthy and rigorous analysis procedure that includes simulating the aircraft at thousands of load cases identified in the certification requirements. Imposing a Global Finite Element Model (GFEM) in this process is computationally very expensive. Hence, Reduced Order Models (ROM) of airframes are commonly employed in the static and dynamic aeroelasticity analyses. ROMs must be simple enough to be analyzed thousands of times during the iterative aeroelastic simulation but sufficiently accurate to have their dynamic characteristics closely matching those of the GFEM within a frequency range of interest. Several Model Order Reduction (MOR) methodologies are available in the literature with the Stick Model (SM) being the preferred methodology adopted by the aerospace industry. A SM is a series of beam elements extending along the airframe elastic axis that offers an intuitive spatial representation of the airframe mass and stiffness distributions, a feature of paramount importance to the development engineers in the aerospace industry. However, due to several approximations and simplifications in the current development process, it is evidently found that SM's are not sufficiently appropriate for dynamic simulations. To overcome such limitation, this thesis presents two approaches for the development of high fidelity stick models. The first approach is based on a Hybrid Stick Model (HSM) representation in which the conventional SM is augmented by a set of structural matrices to account for inaccuracies that might be encountered in the modal performance of the base SM as compared to the GFEM. In the second approach, we solve a design optimization problem in which we optimize the stiffness parameters of the conventional SM to minimize errors in its modal pairs with reference to the GFEM. The final product of the optimization 
problem is an Optimized Stick Model (OSM) with dynamic characteristics that closely matching those of the GFEM within a specified frequency range of interest. Case studies are presented where the HSM and the OSM along with the conventional SM are employed in the dynamic aeroelasticity loads analyses of a Bombardier aircraft platform. The extracted aeroelastic loads are compared against those generated employing the aircraft GFEM. The dynamic characteristics of the ROMs are also assessed based on their modal characteristics using metrics of Modal Assurance Criteria (MAC) and Modal Participation Factors (MPF). Results obtained show that the developed HSM and OSM have superior dynamic characteristics compared to the conventional SM.

Keywords: Aeroelasticity analysis; Stick Model; Model Order Reduction; Design Optimization; Craig Bampton Reduction; Guyan Reduction; Component Mode Synthesis. 


\section{PRefaCe}

The work presented in this thesis, is an original work developed by Paul Vazhayil Thomas, under the supervision of the Prof. Mostafa El Sayed, conforming with all mandatory requirements as stated by Carleton University.

The project is part of the Bombardiers initiative to develop a multidisciplinary framework for optimization of aircraft wing-box. In close collaboration, Bombardier Aerospace provided data relevant to the aeroelastic numerical model, which is used as part of the case studies in chapter 3, 4 and 5. All numerical data provided has been normalized to protect the integrity of our partner's' design.

The work presented in chapter 2 and Chapter 3 is accepted for journal publication titled "Review of Model Order Reduction Methods and their Applications in Aeroelasticity Loads Analysis for Design Optimization of Complex Airframes" in ASCE Journal of Aerospace Engineering, 2018.

In addition, Hybrid Stick Model based reduction methodology presented in Chapter 4 is submitted for publication under title" Development of High Fidelity Reduced Order Hybrid Stick Model for Aircraft Dynamic Aeroelasticity Analysis”. Both articles, listed here, are co-authored by Professor Mostafa El Sayed and Denis Walch of Bombardier Aerospace. 


\section{ACKNOWLEDGEMENTS}

I would like to express my sincere gratitude to my supervisor, Prof. Mostafa El Sayed, for his valuable advice, support, encouragement and guidance throughout the course of this work.

I would like to thank Denis Walch (Engineering Specialist at Bombardier Aerospace) for his relentless support during these two years and his most valuable input into this project.

I am also grateful for the financial support from BOMBARDIER INC., Montreal, in collaboration with CARIC National Forum and MITACS Canada.

I would like to thank my colleagues and friends in the Aircraft Structures and Materials Laboratory for their assistance. Specially, I would like to thank Michelle Guzman and Muhammad Ali for providing me with invaluable insights and comments in my research work. I would also like to thank the other researchers, past and present, who have been in CB6103 lab, for their suggestions and friendship.

Finally, I would like to express my deepest gratitude to my parents Mr. V. M. Thomas and Mrs. Marykutty Thomas and my brother Libni Thomas and my sister in law Mrs. Sibi Libni Thomas for their endless support, and patience. Undoubtedly, the constant encouragement and moral support from my family has helped me become the person I am today. 


\section{TABLE OF CONTENTS}

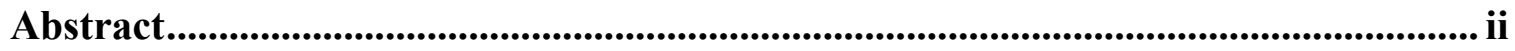

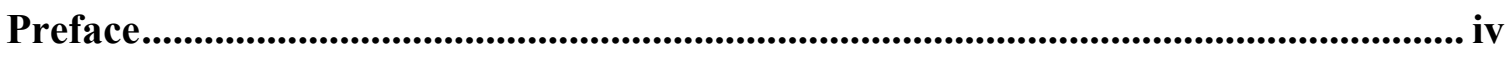

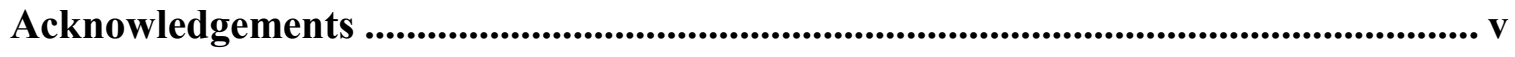

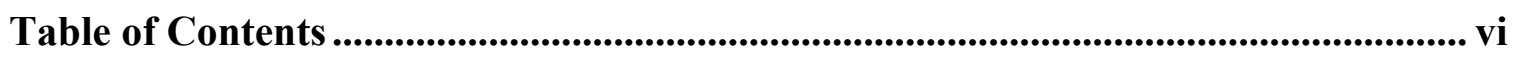

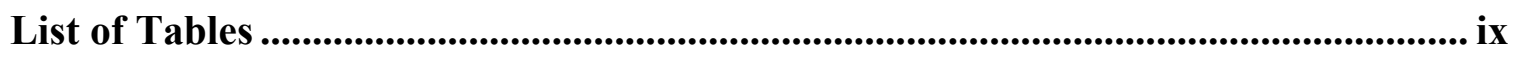

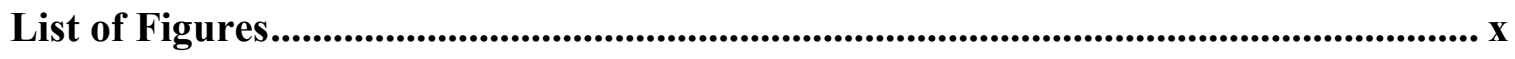

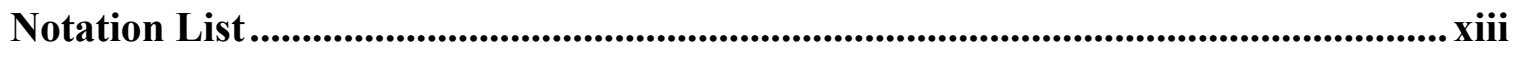

List of Abbreviations ................................................................................................ 1

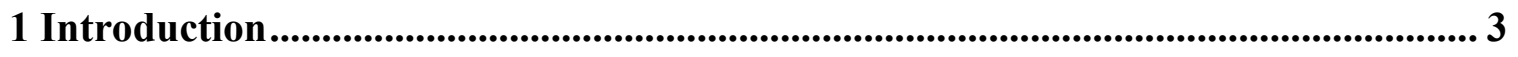

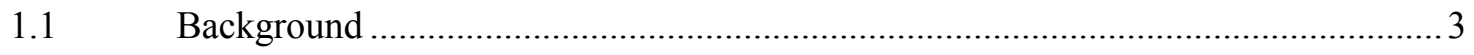

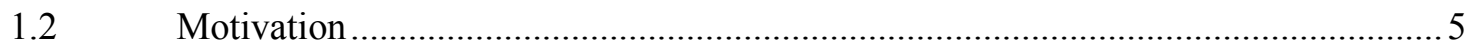

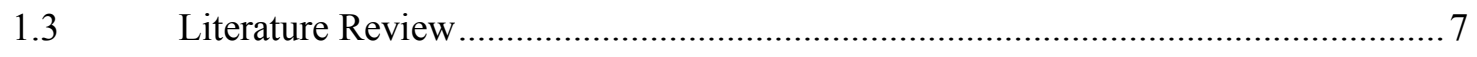

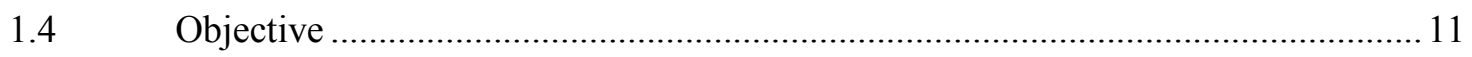

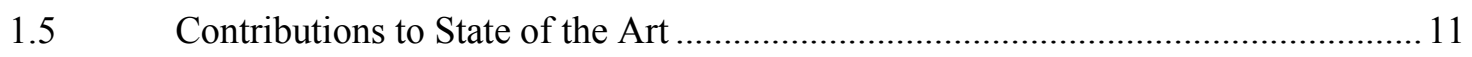

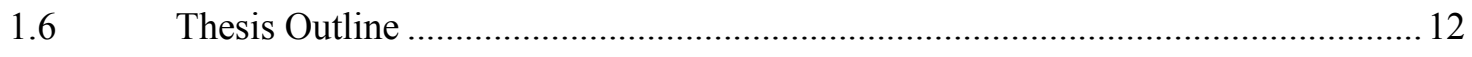

2 Model order reduction Methodologies: Mathematical Framework ........................ 13

2.1 Stick Model Development by Unitary Loading Method ......................................... 13

2.2 Linear Algebraic Matrix-Based Reduction Methodologies ................................. 16

2.2.1 Physical coordinate projection MOR methods ......................................... 18

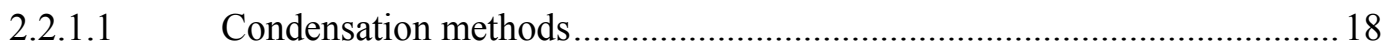

2.2.1.1.1 Guyan-Irons Reduction .............................................................. 19

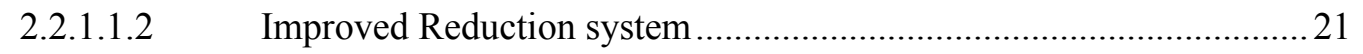

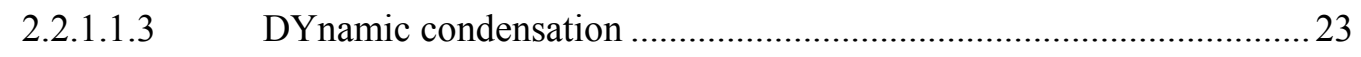

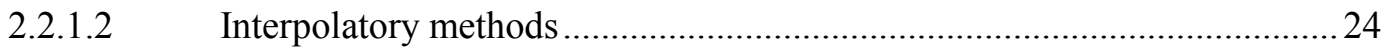


2.2.1.2.1 Derivative based Galerkin projection ............................................25

2.2.1.2.2 Krylov based Galerkin projection ...............................................2 25

2.2.2 Modal coordinate projection based MOR methods ......................................26

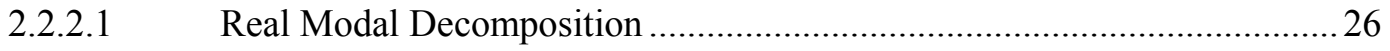

2.2.2.2 Complex Modal Decomposition .............................................................. 27

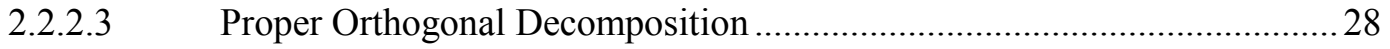

2.2.3 Hybrid coordinate projection based MOR methods ......................................29

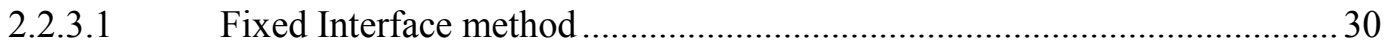

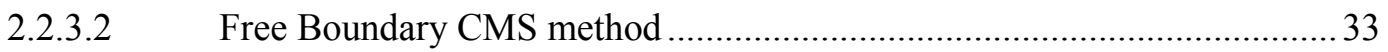

3 Dynamic performance of Model order reduction Methodologies: a Case Study... 35

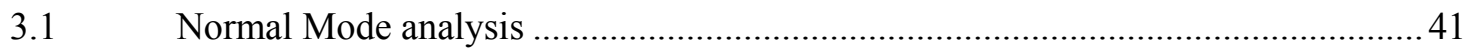

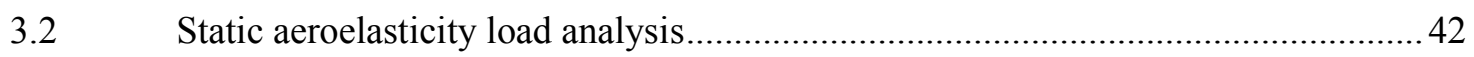

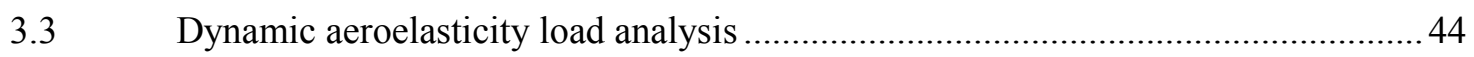

3.3.1 Recovered dynamic load in TDG gust case .............................................. 45

3.3.2 Recovered dynamic load in PSD vertical gust case ................................... 46

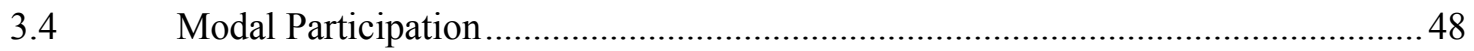

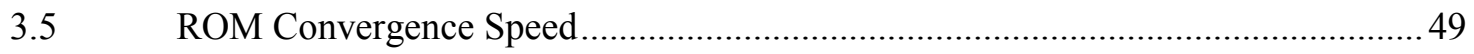

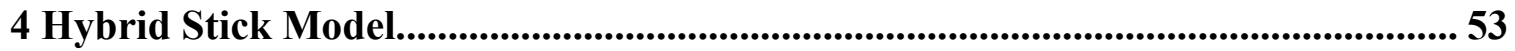

4.1 Residual Matrices of the HSM based on GR ROM .............................................54

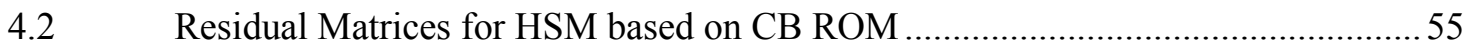

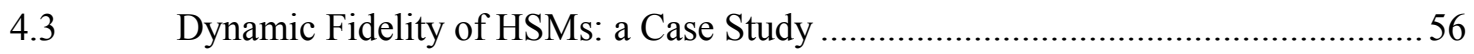

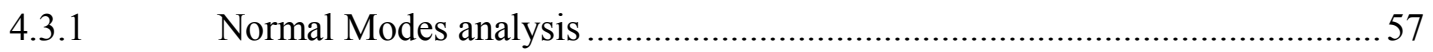

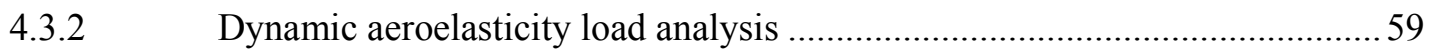

4.3.2.1 Recovered dynamic load in TDG gust case...........................................59

4.3.2.2 Recovered dynamic load in PSD vertical gust case.................................. 60

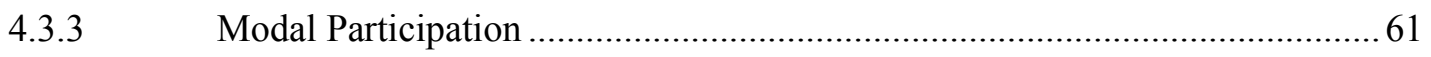


4.4 Handling Capabilities of HSMs

5 Design optimization of aircraft Stick Model ............................................................... 69

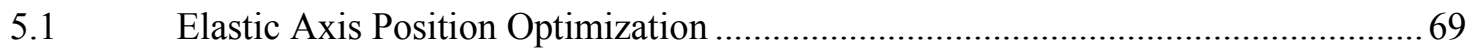

$5.2 \quad$ Stick Model Stiffness Parameters Optimization .................................................. 71

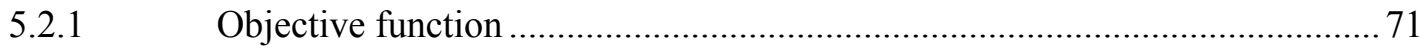

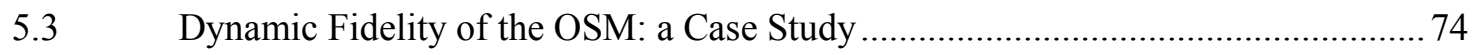

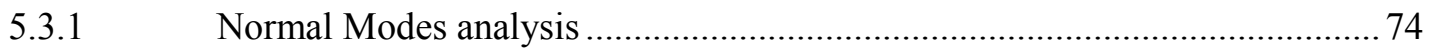

5.3.1 Dynamic aeroelasticity load analysis .................................................... 76

5.3.1.1 Recovered dynamic load in TDG and PSD gust case ..............................76

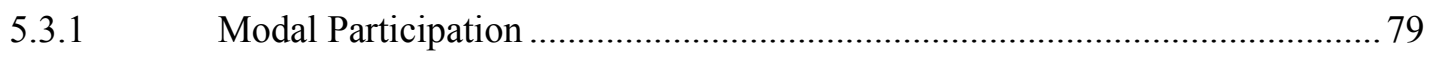

6 Conclusion and Future Work ....................................................................... 81

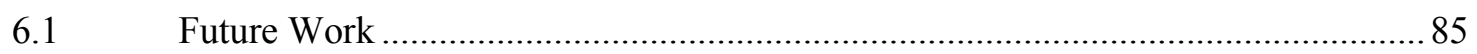

A Appendix: Timoshenko Beam element stiffness matrix ............................... 87

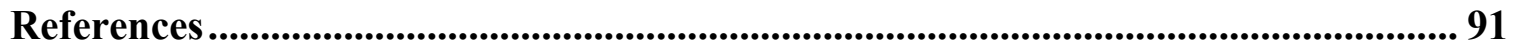




\section{LIST OF TABLES}

Table 3.1 Flight conditions for TDG analysis ....................................................... 45

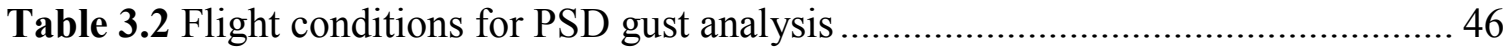

Table 3.3 Properties of Von Karman gust................................................................ 46

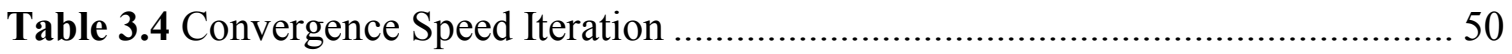

Table 3.5 Summary of RMS error associated with each analysis ............................... 51

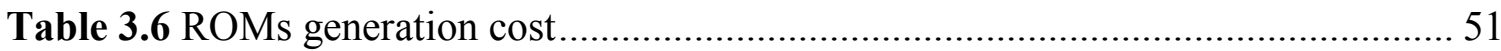

Table 3.7 GFEM and ROMs analysis cost per aeroelasticity iteration ........................ 51

Table 4.1 Summary of RMS error associated with SM and HSM ROMs for each analysis

Table 4.2 GFEM, SM and HSM ROMs analysis cost per aeroelasticity iteration........... 63

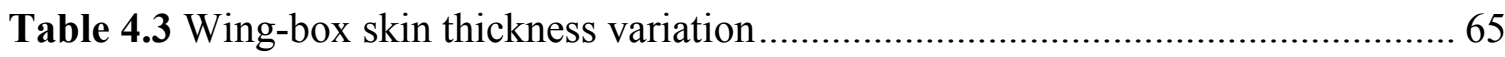

Table 5.1 Summary of RMS error associated with SM and OSM ROMs for each analysis

Table 6.1 Comparison of ROMs based on their compatibility for aeroelastic loads recovery

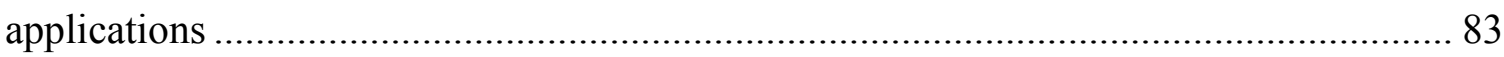




\section{LIST OF FIGURES}

Figure 1.1 Aeroelasticity Optimization loop and loads recovery ................................ 4

Figure 1.2 Three-ring aeroelastic Venn Diagram .............................................. 5

Figure 1.3 Model Order Reduction Techniques........................................................... 7

Figure 2.1 Schematic drawing showing GFEM reduction process to a SM .................. 13

Figure 2.2 GR reduced Stiffness matrix............................................................. 20

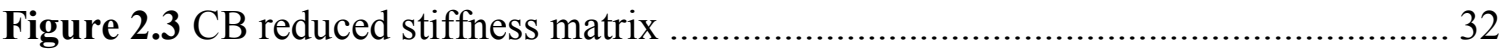

Figure 3.1 Visual representation of a generic aircraft ROMs (SM and Matrix based ROMs)

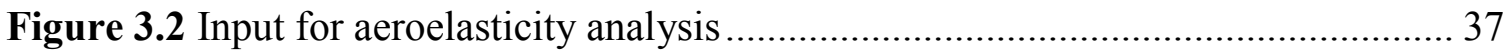

Figure 3.3 Aerodynamic DoFs coupled to the SM structural DoFs in aeroelasticity analysis

Figure 3.4 Lumped mass idealization for aircraft RHS wing-box ............................... 39

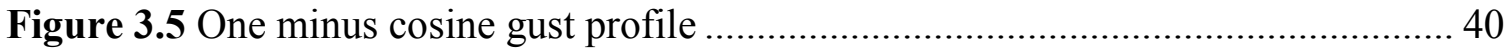

Figure 3.6 Percentage of error involved in the reduced models as compared to GFEM for the first 10 flexible natural frequencies .............................................................. 41

Figure 3.7 Static Aeroelasticity loads recovery highlighting grid points and reference coordinate. 42

Figure 3.8 Comparison of static out of plane shear loads recovered from all ROMs and GFEM 43

Figure 3.9 Comparison of static out-plane bending moment recovered from all ROMs and GFEM 43

Figure 3.10 Reduced model dynamic loads recovery grid point and coordinate. 44 
Figure 3.11 Comparison of out of plane bending moment recovered from all six ROMs and GFEM for TDG case 46

Figure 3.12 Comparison frequency response function (Magnitude) for all six ROMs and GFEM 47

Figure 3.13 Comparison frequency response function (phase) for all six ROMs and GFEM 48

Figure 3.14 Comparison of Modal Participation Factor for all six ROMs with GFEM .. 48 Figure 3.15 Percentage of error in frequency response of ROMs with respect to number of their retained grid points as compared to GFEM counterpart...................................... 50

Figure 4.1 Visual representation of a generic aircraft HSM ROM ............................ 56

Figure 4.2 Percentage of error involved in the SM and HSM ROMs as compared to GFEM

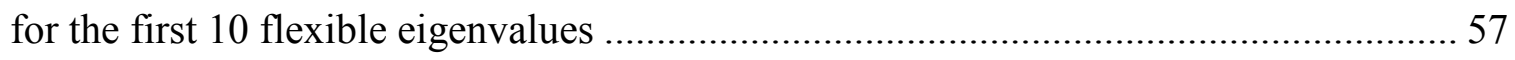

Figure 4.3 Normal modes comparison between SM ROM and GFEM based on MAC.. 58 Figure 4.4 Normal modes comparison between HSMs and GFEM based on MAC ....... 58 Figure 4.5 Comparison of out of plane bending moment recovered from SM, HSM ROMs and GFEM. 60

Figure 4.6 Comparison frequency response function (Magnitude) for SM, HSM ROMs and GFEM 61

Figure 4.7 Comparison frequency response function (phase) for SM, HSM ROMs and GFEM 61

Figure 4.8 Comparison of Modal Participation Factor for SM and HSM ROMs with GFEM 62

Figure 4.9 Skin thickness variation location for both GFEM and HCB ROM. 64 
Figure 4.10 RMS error associated with the HCB ROM eigenvalues compared to GFEM in all iterations. 65

Figure 4.11 Normal modes comparison based on MAC for HCB and GFEM for all iterations. 66

Figure 4.12 Comparison of out of plane bending moment (TDG case) for GFEM and HCB in iteration 1 67

Figure 4.13 Comparison of out of plane bending moment (TDG case) for GFEM and HCB in iteration 4 67

Figure 4.14 Comparison of the FRF (PSD case) for GFEM and HCB in iteration 1 ...... 68

Figure 4.15 Comparison of the FRF (PSD case) for GFEM and HCB in iteration 4 ...... 68

Figure 5.1 Wing-box elastic axis determination 71

Figure 5.2 Percentage of error in SM and OSM ROMs as compared to GFEM for the first 10 flexible eigenvalues 75

Figure 5.3 Normal modes comparison between OSM ROM and GFEM based on MAC75

Figure 5.4 OSM and SM dynamic loads recovery grid point and coordinate 76

Figure 5.5 Comparison of out of plane bending moment recovered from SM, OSM and GFEM 77

Figure 5.6 Comparison frequency response function (Magnitude) for SM, OSM and GFEM 78

Figure 5.7 Comparison frequency response function (phase) for SM, OSM and GFEM 78 Figure 5.8 Comparison of Modal Participation Factor for SM, OSM and GFEM ......... 79

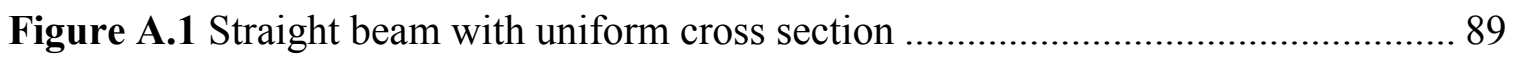

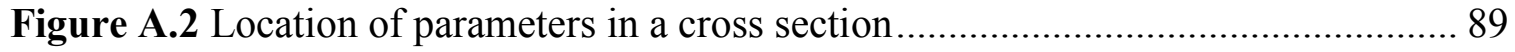


A State space matrix

A Cross sectional area

B State space input matrix

D Damping matrix

E Young's Modulus of material

G Shear Modulus

H n by n matrix for Krylov subspace

I Identity matrix

$I_{y} \& I_{z} \quad$ Second moment of area along $\mathrm{Y}$ and $\mathrm{Z}$ directions

$J_{x} \quad$ Torsional moment of inertia

K Stiffness matrix

$K_{Y} \& K_{\mathrm{Z}} \quad$ Shear factor in in $\mathrm{Y}$ and $\mathrm{Z}$ directions

$L \quad$ Length of one bay

$L_{g} \quad$ Length of the gust

$L_{t} \quad$ Characteristic scale wavelength of the turbulence

M Mass matrix

$N \quad$ Dimension of the full-order model

$P \quad$ Total frequency sampling points

$\boldsymbol{R} \quad$ Residual Matrix

$\mathbb{R} \quad$ Real axis 


$\begin{array}{ll}\mathbf{T} & \text { Reduced order basis vectors } \\ T & \text { Matrix transpose } \\ V & \text { Flight speed } \\ V_{g} & \text { Gust Velocity } \\ V_{g 0} & \text { Maximum peak value of the gust }\end{array}$

\section{Minuscule letters Definitions}

$\begin{array}{cl}\mathbf{b} & \text { Vector for Krylov subspace } \\ \boldsymbol{c}_{r} & \text { Penalty cost-value vector } \\ e & \text { Percentage of error } \\ \mathbf{f} & \text { Applied force vector } \\ f(\mathbf{y}) & \text { Objective function } \\ n_{o} & \left.\text { Omitted DoFs (N }-n_{r}\right) \\ n_{i} & \text { Number of interior generalized coordinates for CB } \\ n_{r} & \text { Retained Degrees of Freedom (DoFs) } \\ n_{i-d} & \text { Number of dominant normal modes included in CB reduction } \\ n_{i-w} & \text { Number of weak normal modes neglected in CB reduction } \\ \boldsymbol{q} & \text { Generalized coordinate } \\ q & \text { Total number of beam elements in the SM ROM } \\ u_{g} & \text { Position of aircraft in the spatial representation of the gust } \\ \mathbf{x} & \text { Grid displacement vector in physical coordinate } \\ \dot{\mathbf{x}} & \text { Velocity in physical coordinate }\end{array}$


$\ddot{\mathbf{x}}$

$\mathbf{y}$

Z

Greek letters

$\delta$

$\theta$

$\omega \quad$ Circular frequency in $\mathrm{rad} / \mathrm{s}$

ф Real Modal matrix

$\boldsymbol{\Psi} \quad$ Linear transformation matrix

$\Lambda \quad$ Diagonal eigenvalue matrix

$\Phi_{g}(\Omega) \quad$ Random air velocity as a function of scaled frequency

$\sigma_{g} \quad$ Root Mean Square turbulence velocity

$\boldsymbol{\lambda}$ Diagonal eigenvalue matrix

$\boldsymbol{\eta} \quad$ Complex generalized coordinate

$\mathcal{K}_{j} \quad \mathrm{j}^{\text {th }}$ order Krylov subspace

$\bar{\Psi} \quad$ Left eigenvector matrix

$\bar{\phi} \quad$ Right eigenvector matrix

$\Phi_{i}, \Phi_{o} \quad$ Input and output PSD function.

$\Phi \quad$ Eigenvector

$\xi_{\min }, \xi_{\max } \quad$ Bounds for shear center determination along y axis 


\section{LIST OF ABBREVIATIONS}

\begin{tabular}{|c|c|}
\hline CAD & Computer Aided Design \\
\hline $\mathrm{CB}$ & Craig Bampton \\
\hline CMD & Complex Modal Decomposition \\
\hline CMS & Component Mode Synthesis \\
\hline DA & Deformation Approach \\
\hline DGP & Derivative based Galerkin Projection \\
\hline DLM & Doublet Lattice Method \\
\hline DoF & Degrees of Freedom \\
\hline DY & Dynamic condensation \\
\hline EA & Elastic Axis \\
\hline $\mathrm{FF}$ & Free-Free Boundary Method \\
\hline FRF & Frequency Response Function \\
\hline GFEM & Global Finite Element Model \\
\hline GR & Guyan Reduction \\
\hline $\mathrm{HCB}$ & Hybrid stick model based on Craig Bampton \\
\hline HGR & Hybrid stick model based on Guyan Reduction \\
\hline HSM & Hybrid Stick Model \\
\hline IRS & Improved Reduction System \\
\hline KGP & Krylov based Galerkin Projection \\
\hline LHS & Left Hand Side \\
\hline MA & Momentum Approach \\
\hline
\end{tabular}




$\begin{array}{ll}\text { MAC } & \text { Modal Assurance Criteria } \\ \text { MPF } & \text { Modal Participation Factor } \\ \text { OSM } & \text { Modal Order Reduction } \\ \text { POD } & \text { Proper Orthogonal Decomposition } \\ \text { PSD } & \text { Power Spectral Density } \\ \text { RHS } & \text { Right Hand Side } \\ \text { RMD } & \text { Real Modal Decomposition } \\ \text { RMS } & \text { Root Mean Square } \\ \text { ROM } & \text { Reduced Order Model } \\ \text { SM } & \text { Stick Model } \\ \text { TDG } & \text { Tuned Discrete Gust }\end{array}$




\section{Introduction}

\subsection{Background}

Aeroelasticity loads analysis play an important part across much of the design and development of an aircraft. The design of aircraft is a prolonged process which consist of four stages, namely, conceptual design phase, preliminary design phase, detailed design phase and validation and certification design phase [1]. Much of the activity that occurs during conceptual, preliminary and detailed design phases involves the constrained optimization of the aircraft model. The aircraft design optimization is an iterative process and the aircraft design cycle is repeated until a balance is achieved between various design criteria. Each iteration in optimization results in the modification of size and geometry of the aircraft structural components. This in turn, changes the static and dynamic aeroelastic behavior of the aircraft. Hence, a full aeroelastic loads analysis is required to identify the new critical loads envelope.

Identification of aircraft critical loads requires a lengthy and rigorous analysis procedure that includes simulating the aircraft at thousands of load cases identified in the certification requirements $[1,2,3,4,6]$. It is computationally very expensive to use a GFEM for this process, hence simplified finite element models, also known as ROMs, are commonly used particularly in the dynamic aeroelasticity loads analysis. ROMs must have dynamic characteristics closely matching those of the GFEM within a frequency range of interest.

A block diagram of the aeroelasticity loads analysis employed in aircraft iterative design optimization process is shown in Figure 1.1. Aircraft aeroelastic model represents 
the coupling between the structural and the aerodynamic disciplines. The GFEM is prepared once the aircraft geometry becomes available which is then condensed into a ROM during aeroelastic loads analyses. GFEM is a low fidelity and coarse meshed finite element model, representing the airframe structure, formed using a collection of shell and bar elements. On the other hand, the aerodynamic model, based on paneling technique, such as Doublet Lattice Method (DLM), is superimposed into the structural ROM to represent the aerodynamic forces due to fluid-structure interaction. The structural and the aerodynamic models are coupled through splining system, which relates displacements of the structural grids to those of the aerodynamic model. Thereafter, aeroelasticity analyses are conducted and the critical loads envelope is constructed through monitoring the different load cases analyzed.

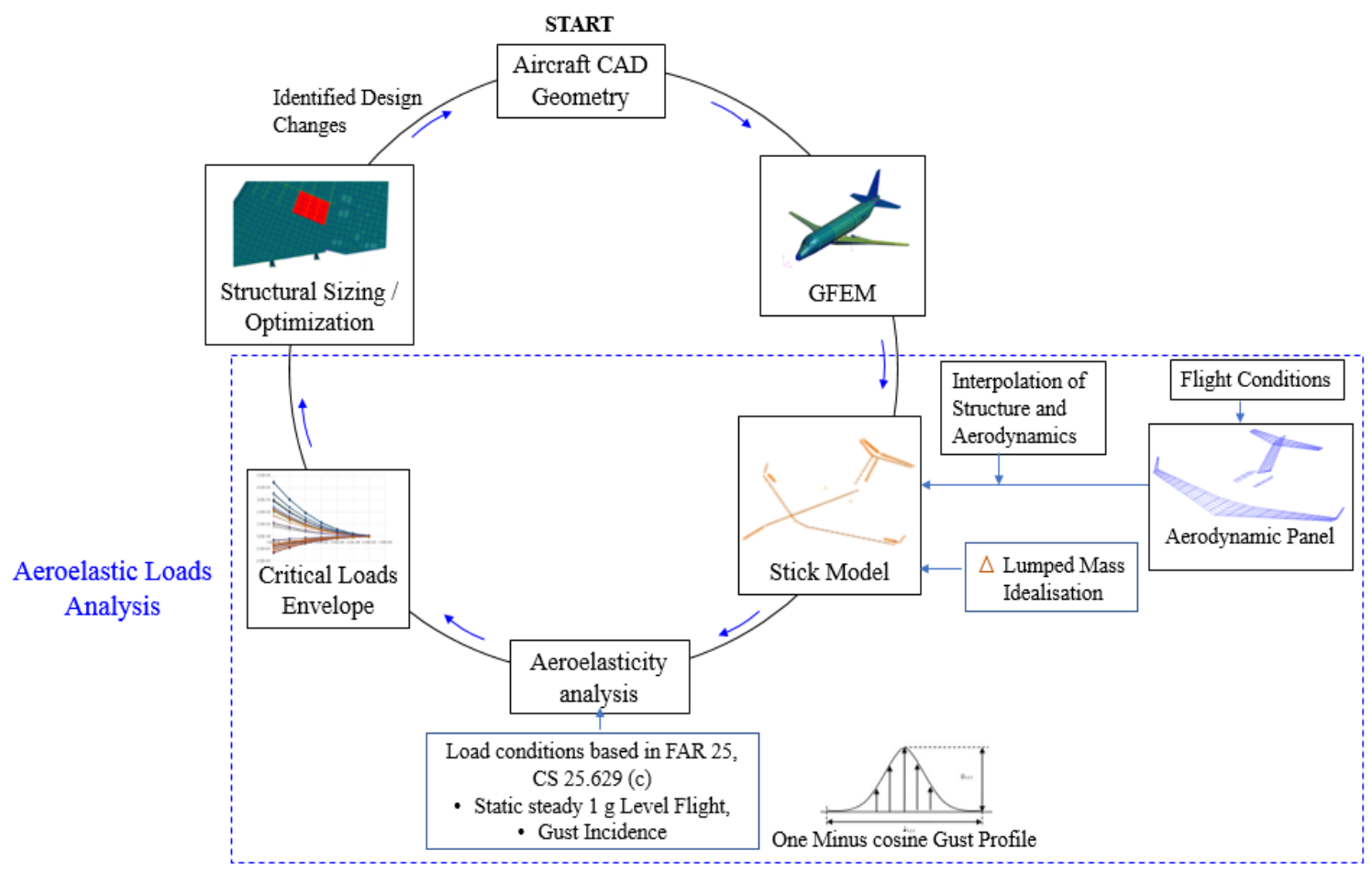

Figure 1.1 Aeroelasticity Optimization loop and loads recovery 
Aircraft aeroelastic loads arise due to the interaction between elastic, inertial and aerodynamic forces [1]. This interdisciplinary interaction is represented in the form of Venn diagram, as shown in Figure 1.2. In general, aeroelastic loads in flight can be divided into Static and Dynamic components. The former stems from the aerodynamic pressure distribution along the aircraft outer mold line while the latter is due to the dynamic response of the aircraft arising from external excitations such as gust turbulence [1,2].

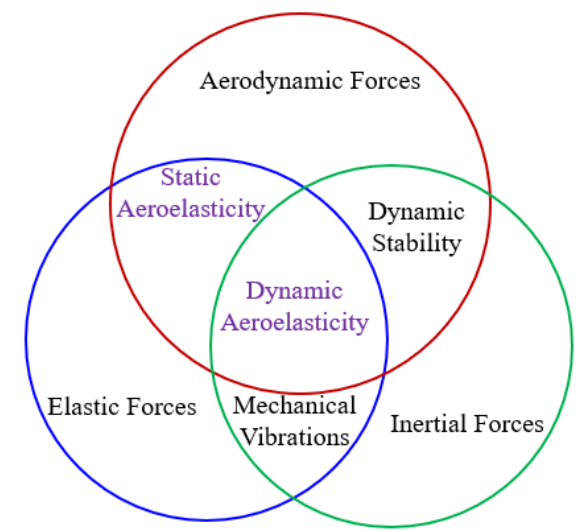

Figure 1.2 Three-ring aeroelastic Venn Diagram

\subsection{Motivation}

Several MOR methodologies are available in the literature [7-86]. The SM developed by unitary loading approach $[9,10]$ is the conventional ROM used in the aerospace industry. A SM is a series of beam elements oriented along the aircraft elastic axis that supposedly represents the dynamic characteristics of the detailed GFEM of the airframe. It offers an intuitive spatial representation of the airframe mass and stiffness distributions. This feature enables loads analysis engineers to study the sensitivity of aircraft performance metrics to different derivatives of airframe structural design without the need of consulting with the structural engineers with each airframe variation being considered. 
However, the accuracy of SM highly depends on the proper orientation of the airframe structural principal axes with the torsional axis aligned along the aircraft elastic axis at each airframe bay. The determination of the elastic axis of a detailed airframe structure is a fairly complicated task especially in the case of wing-box structures. Here, approximate methods are typically used in the aerospace industry to orient the principal coordinates at each wing bay to generate the equivalent beam stiffness properties [17]. This results in several inaccuracies in the SM beam properties with those errors being significant in beam stiffness properties extracted from the aircraft bays located towards the inboard side of the wing [9]. Moreover, the conventional stick modeling development process involves the assumptions that cross-section of the SM beam elements are constant with no bending and torsional coupling involved because of the zero offset between the centroid and the shear center. Hence, it is found that the SM lacks the capability to represent the coupled bending and torsional dynamic behaviors of the GFEM. Furthermore, it is common that a SM based on unitary loading method overestimates the GFEM stiffness for the out of plane bending and the torsional modes [13]. Accordingly, it is found that the SM is not adequately capable of representing the complex dynamics behavior of airframes [9]. As a correction measure, a validation of the SM ROM in reference to the GFEM is performed for each major stiffness variation. This is followed by a process to minimize the dynamic behavior mismatch between the SM and the GFEM by adjusting the stick beam properties, which can be a time consuming process in most cases [13]. On the other hand, matrix-based ROMs, such as Guyan [18] and Craig Bampton [25] reduced models, have shown excellent dynamic performance as compared to their GFEM counterparts. Nevertheless, matrix reduction methods do not offer the intuitive flexibility sought by aircraft development engineers. 
Hence, there is a pressing need to generate a beam element based ROM which have dynamic characteristics closely matching to the GFEM.

\subsection{Literature Review}

Two forms of ROMs are available in the literature, namely, the stick and matrix-based ROMs as shown in Figure 1.3 [7].

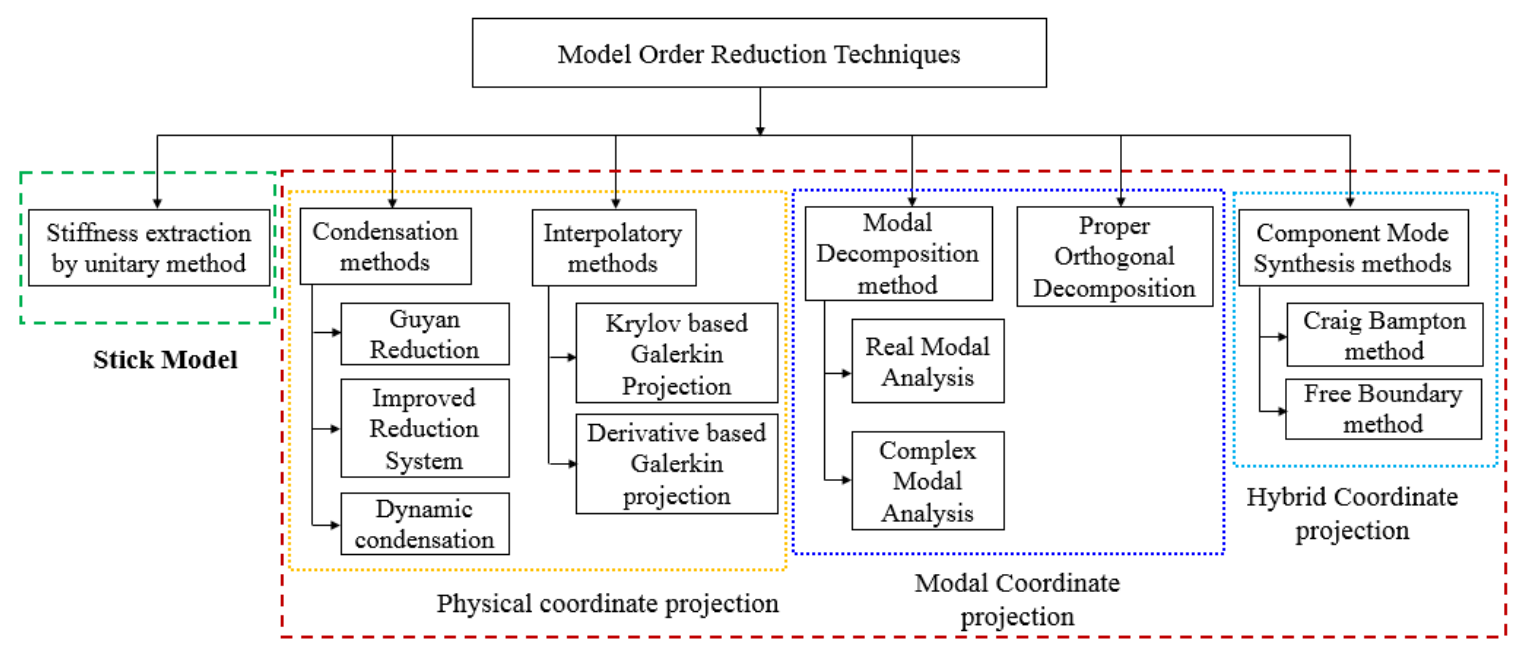

Linear Algebraic Matrix Reduction

Figure 1.3 Model Order Reduction Techniques

Several SM development techniques are discussed in the literature. ElSayed et al. compared different SM reduction methods commonly used in the aerospace industry for multidisciplinary design optimization [10]. He also proposed a methodology to develop SM employing a modulated 3D rigid rotational mapping to match the spatial deformations of the SM with those of the elastic axis of the GFEM. The simplest technique for SM development would be an analytical approach to determine the beam stiffness properties from the airframe cross section geometry once the aircraft CAD model or GFEM is prepared [11]. Another technique involves the extraction of beam stiffness properties using 
unitary loading method $[12,13]$. Here, equivalent beam stiffness properties of the SM along the aircraft elastic axis are estimated from the deformations corresponding to the application of unit loading moments at the free end of cantilevered singled bays of the aircraft GFEM. Hashemi-Kia and Toossi [14] developed a similar reduction technique in which the stiffness properties are extracted by applying unit deformations at one free end assuming cantilevered condition. Also, Riccardo [15] introduced another SM methodology where the beam constitutive laws are used for the generation of the beam stiffness properties from the GFEM while condensed mass properties are generated using the mass development tool in MSC PATRAN [16].

Matrix-based MOR is an alternative approach for the development of airframe ROMs. A Matrix-based MOR method is a Galerkin projection of the mass and stiffness matrices of the GFEM onto a carefully chosen reduced order basis subspace. As shown in Figure 1.3, matrix-based reduction methodologies are classified into three categories based on the type of basis vectors identifying the reduced projection subspace, namely, physical, modal and hybrid (combination of physical and modal basis) projection methods. Several linear algebraic MOR methodologies are available in the literature [18-53]. Matrix-based reduction methods within the category of physical projection approach include the condensation and the interpolatory techniques. Prominent MOR methods based on condensation technique include Guyan-Irons Reduction (GR) [18, 19], Improved Reduction System (IRS) [22] and DYnamic condensation (DY) methods [24]. Guyan and Irons proposed the static condensation method (commonly known as Guyan method) which involves the formation of reduced subspace using static constraint modes, ignoring the inertial terms. O'Callaghan [22] introduced a new condensation method called IRS to 
include a first order correction for mass effects at the omitted DoF for the static condensation. DY [24] is introduced as an improvement for the GR method where the mass matrix is also considered in the development of the reduced subspace of the ROM. Various studies have shown that the accuracy of these condensation methods can be improved by using an iterative scheme to form the reduced order basis subspace [54-63].

On the other hand, interpolatory MOR technique [35-44] relies on developing the detailed dynamic system response at the full spectrum of frequencies of interest through interpolation of the system response function and its derivatives at a subset of identified sampling frequencies. Taylor series and Pade approximation [64] are the two common methods used for constructing the interpolants of displacement function within this MOR technique. In other words, Interpolatory methods are approximation methods with the objective to alleviate the cost associated with the frequency sweep analysis. The MOR methods based on this technique include Krylov-based Galerkin Projection (KGP) and the Derivative based Galerkin Projection (DGP) [49]. DGP method is based on forming the projection subspace using the response function derivatives whereas KGP constructs the reduced subspace employing Krylov subspace that span the derivatives of the response function.

Modal decomposition methods are based on modal truncation approach where reduction transformation matrices are formed using the normal modes within the frequency range of interest [65]. Here, a Galerkin projection is conducted to transform the full dynamical system to the reduced modal coordinate basis in order to approximate the frequency response function. This category includes Real Modal Decomposition (RMD) and Complex Modal Decomposition (CMD). 
Proper Orthogonal Decomposition (POD) is another popular MOR method in structural dynamics field [66 - 69] which comes under modal coordinate projection approach. This reduction methodology involves the formation of a simple covariance matrix using the ensemble of displacement vectors corresponding to a set of frequencies within the frequency range of interest. Then, an orthogonal transformation of the high dimensional equation of motion is performed to the reduced basis indicated by the eigenvectors of the covariance matrix.

Component mode synthesis is the third class of MOR methodologies reviewed. This technique relies on a combination of physical and modal basis vectors to form the reduced subspace. In 1965, Hurty [70] introduced the concept of component mode synthesis (CMS) using sub-structuring for large dynamical structures. Since then, various CMS methods are introduced in the last five decades [25-34] which have gone through several enhancements [71-77]. Component mode synthesis involves partitioning a large dynamical system into several subsystems. The displacement of each subsystems are expressed in terms of a combination of generalized and physical coordinates (or hybrid coordinates) using a truncated set of normal modes or a combination of normal modes and static modes. Then the reduced mass and stiffness matrices are formed by the matrix projection onto the reduced subspace formed by the displacement modes. The reduced matrices are assembled for all the subsystems through interface coupling and the dynamic response of the total system is solved. Depending on the interface coupling conditions, CMS methods are categorized into two types, namely, fixed $[25-29,71,72]$ and free interface methods [30$32,34]$. 


\subsection{Objective}

This thesis presents two high fidelity ROM methodologies, namely, HSM and OSM, to be employed in the aeroelasticity loads analysis. The former is an improved form of the conventional SM which is developed by augmenting the SM ROM with a residual mass and stiffness matrices that account for the dynamic imparity between the SM and the GFEM as well as the coupling of Degrees of Freedom (DoFs) commonly ignored by the simplified SM representation. The latter, on the other hand, is generated by solving a constrained optimization problem to match a set of eigenvalues and eigenvectors of the GFEM within a frequency range of interest. A case study is presented where the HSM and OSM ROMs developed, along with their conventional SM counterpart, are employed in the dynamic aeroelasticity loads analyses of a Bombardier aircraft platform. Using monitor points method, the extracted aeroelastic loads employing the ROMs are compared against those generated employing the aircraft GFEM. The dynamic characteristics of the ROMs are also assessed based on their modal characteristics using MAC along with their loads MPFs. Results obtained show that the developed HSM and OSM ROMs have superior dynamic characteristics compared to the conventional SM.

\subsection{Contributions to State of the Art}

The author has made following contributions to the state of the art,

1. Reviewing the applicability of 10 matrix based MOR methodologies along with the conventional stick model for the aeroelasticity loads analysis. Generation of in house tools for the MOR methods reviewed. All the generated ROMs are compared in terms of various loads analysis criteria and the best MOR methods are identified. 
This review of all the MOR methodologies is published as a journal paper [7].

2. Proposed a Hybrid Stick Modelling Approach for the high-fidelity ROMs in aeroelasticity loads analyses. This work is submitted for journal publication, which is currently in review [8].

3. Design Optimization of SM ROM to match a set of eigenvalues and eigenvectors of the GFEM. The generated OSM have significant improvement in the dynamic characteristics as compared to the base SM ROM.

\subsection{Thesis Outline}

This thesis is organized in six chapters. After the introduction, a detailed mathematical description of conventional SM and 10 matrix based MOR methodologies available in the literature are presented in chapter 2 . Thereafter, in chapter 3 , an exhaustive case study employing the conventional SM and the matrix-based methods reviewed are employed in the static and dynamic aeroelasticity loads analyses of a Bombardier aircraft platform. Then, a new high fidelity reduced order modelling methodology based on a HSM representation approach is presented, along with a case study, in chapter 4. Chapter 5 presents OSM generation using a design optimization of conventional SM to match a set of eigenvalues and eigenvectors of the GFEM along with a case study. This thesis concludes in chapter 6 , with a discussion highlighting the important attributes of the all reviewed methodologies along with the proposed two MORs methods in the aeroelasticity loads analysis. We also discuss potential future work in the last section of chapter 6 . 


\section{Model order reduction Methodologies: Mathematical Framework}

This chapter provides a detailed mathematical description of the common MOR methodologies available in the literature, which include SM ROM based on "Unitary Loading Method" and 10 matrix based MOR methodologies as shown in Figure 1.3.

\subsection{Stick Model Development by Unitary Loading Method}

In this method, the static response of the GFEM to independently applied unit forces and moments are used to compute the stiffness properties of the SM $[12,13]$. Extracted stiffness properties are represented by a set of first order prismatic Timoshenko beam elements [109] with two nodes extending along the airframe elastic axis.

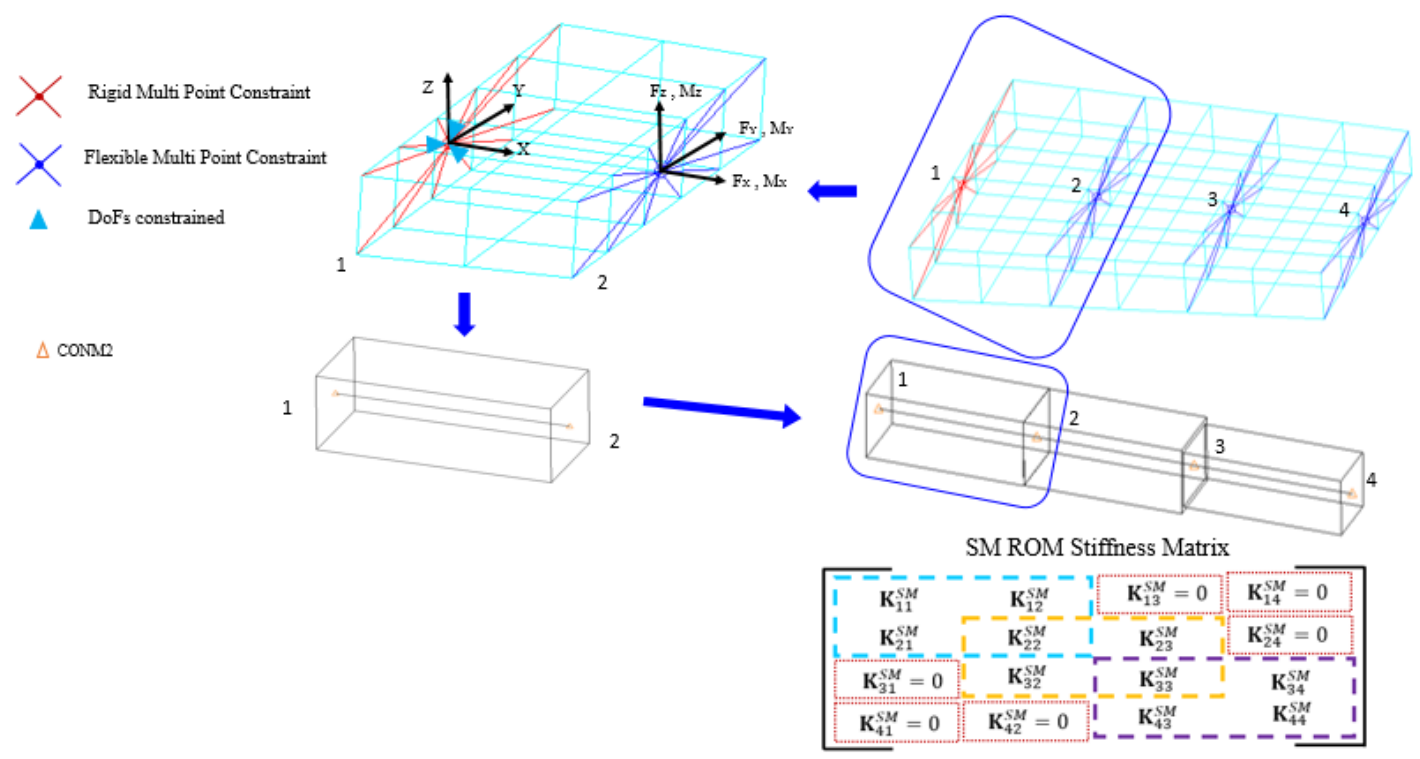

Figure 2.1 Schematic drawing showing GFEM reduction process to a SM

Figure 2.1 shows a schematic drawing that illustrates the SM stiffness extraction process for the GFEM of 3 wing-box bays of an aircraft. A wing bay is the segment of the 
GFEM extending between two consecutive wing stations. The stiffness extraction process is conducted for each wing-box bay which is replaced by a single beam element within the SM ROM. Here, the shear centers of the cross-sections at the two ends of a single wingbox bay are located and a local reference coordinate system is identified. The defined coordinate system is assumed as a principal coordinate system for the wing-box bay structure with its torsional axis extending along the line connecting the predefined shear centers at the two ends of the wing-box bay while the first principal bending axis is assumed along the section airfoil chord line, as shown in Figure 2.1. A cantilevered boundary condition is assumed with the inboard end, 1, is fixed. Six load cases involving unit forces and moments are applied at the shear center of the free end, 2, and the stiffness properties for the beam element representing the wing bay are computed as:

$$
A_{1 \rightarrow 2}=\frac{L_{1-2}}{E\left|\delta_{2-1}\right|_{x}}
$$

where $A_{1 \rightarrow 2}$ is the equivalent cross sectional area, $L_{1-2}$ is the bay length, $\left|\delta_{2-1}\right|_{x}$ is the axial elongation due to the applied unit load along the x-axis at end 2 and $E$ is the material Young's modulus.

Similarly, the shear factors along the y - and the z - directions, $K_{y}$ and $K_{z}$, respectively, are computed as:

$$
\left(K_{y}\right)_{l \rightarrow 2}=\frac{L_{1-2}}{G A_{l \rightarrow 2}\left|\delta_{2-1}\right|_{y}}
$$




$$
\left(K_{z}\right)_{1 \rightarrow 2}=\frac{L_{1-2}}{G A_{1 \rightarrow 2}\left|\delta_{2-1}\right|_{z}}
$$

where $\left|\delta_{2-1}\right|_{y}$ and $\left|\delta_{2-1}\right|_{z}$ denote, respectively, the translational deformation in y- and zdirections due to applied unit forces at end 2 and $G$ is the material shear modulus.

Moments of inertia of the SM beam element are computed using the rotational deformations corresponding to the application of unit moments in the same manner as described before. The equivalent bending moments of inertia $\left(I_{y}\right)_{1 \rightarrow 2}$ and $\left(I_{z}\right)_{1 \rightarrow 2}$, in the yand z- directions respectively, as well as the equivalent torsional moment of inertia, $\left(J_{x}\right)_{1 \rightarrow 2}$ in the $\mathrm{x}$-direction, are computed as:

$$
\begin{aligned}
& \left(I_{y}\right)_{1 \rightarrow 2}=\frac{L_{1-2}}{E\left|\theta_{2-1}\right|_{y}} \\
& \left(I_{z}\right)_{1 \rightarrow 2}=\frac{L_{1-2}}{E\left|\theta_{2-1}\right|_{z}} \\
& \left(J_{x}\right)_{1 \rightarrow 2}=\frac{L_{1-2}}{G\left|\theta_{2-1}\right|_{x}}
\end{aligned}
$$

where $\left|\theta_{2-1}\right|_{x},\left|\theta_{2-1}\right|_{y}$ and $\left|\theta_{2-1}\right|_{z}$ are the angular deformation along x-, y- and zdirections at end 2 , respectively.

A schematic drawing of the assembled stiffness matrix of the SM ROM representing the 3 bays of the wing-box is also shown in Figure 2.1. It can be noted that the SM ROM ignores the coupling between DoFs of the grid points that do not belong to the same finite 
element. This can be seen in the matrix entries $\mathrm{K}_{13}^{S M}, \mathrm{~K}_{14}^{S M}$ and $\mathrm{K}_{24}^{S M}$ as all those terms have the value of zero. It can also be seen that the SM ROM offers a convenient spatial representation of the airframe mass and stiffness distribution, a feature that is required in the aerospace industry as it ease operations for different development groups.

It should be noted that the stick model developed using this methodology is suitable for static analysis. To employ this model in dynamic analysis, lumped mass properties are added at the defined airframe stations. Refer to chapter $3 \mathrm{~b}$ for lumped mass idealization of GFEM.

\subsection{Linear Algebraic Matrix-Based Reduction Methodologies}

Frequency response analysis in structural dynamics usually requires solving a second order equation of motion representing the dynamic system. In a discretized finite element formulation, such equation of motion can be written as,

$$
-\omega^{2} \mathbf{M} \mathbf{x}+\mathrm{i} \omega \mathbf{D} \mathbf{x}+\mathbf{K} \mathbf{x}=\mathbf{f}
$$

where $\mathbf{M}, \mathbf{D}$ and $\mathbf{K} \in \mathbb{R}^{N \times N}$ are the mass, damping and stiffness matrices. $\mathbf{X}$ and $\mathbf{f} \in \mathbb{R}^{N \times 1}$ are the displacement and applied load vectors. Also $\omega$ is the system frequency and $i=\sqrt{-1}$ is the complex number.

Employing Eq. (2.7) for the analysis of large GFEM is computationally very expensive, hence, MOR techniques are commonly considered. Linear algebraic matrixbased MOR methodologies are centered on the idea of forming a transformation matrix, $\mathbf{T}$, that can map a reduced set of DoFs of the ROM to those of the detailed GFEM, such that: 


$$
\mathbf{x} \cong \mathbf{T} \mathbf{x}_{r}
$$

where subscript " $r$ " denotes the reduced set of DoFs, $\mathbf{x}_{r} \in \mathbb{R}^{n_{r} \times 1}$ is the response of the reduced system while $\mathbf{T} \in \mathbb{R}^{N \times n_{r}}$ is the transformation matrix formed by the concatenation of the independent basis vectors of the reduced subspace.

Mass and stiffness matrices as well as applied force vector of the GFEM are projected onto the defined reduced subspace using a Galerkin projection. The resulting reduced equation of motion can be written as,

$$
-\omega^{2} \mathbf{M}_{r} \mathbf{x}_{r}+\mathbf{K}_{r} \mathbf{x}_{r}=\mathbf{f}_{r}
$$

where $\mathbf{M}_{r}, \mathbf{K}_{r} \in \mathbb{R}^{n_{r} \times n_{r}}, \mathbf{x}_{r} \in \mathbb{R}^{n_{r} \times 1}$ and $\mathbf{f}_{r} \in \mathbb{R}^{n_{r} \times 1}$. The reduced mass and stiffness matrices as well as the applied force vector are formulated, respectively, as $\mathbf{M}_{r}=\mathbf{T}^{T} \mathbf{M T}$, $\mathbf{K}_{r}=\mathbf{T}^{T} \mathbf{K T}$ and $\mathbf{f}_{r}=\mathbf{T}^{T} \mathbf{f}$

It should be noted that, modal damping is normally considered in dynamic aeroelasticity analysis. Accordingly, a simplified undamped version of Eq. (2.7) is adopted for the development of ROMs in this chapter. When necessary, damping is introduced through proportional damping formulations.

All linear algebraic matrix reduction methods vary in the formation of the transformation matrix, $\mathbf{T}$, of the reduced subspace. We classify matrix-based MOR methodologies into three categories based on the type of basis vectors identifying their reduced projection subspace, namely, physical, modal and hybrid (combination of physical and modal basis) projection methods. Below is a brief description of the commonly used methodologies under this MOR category. 


\subsubsection{Physical coordinate projection MOR methods}

This section reviews the basic formulations of the linear algebraic MOR methodologies based on Galerkin projection onto a reduced coordinate basis subspace formed in the physical space of the GFEM. These methodologies include the condensation [48] and the interpolatory [49] methods.

\subsubsection{Condensation methods}

Condensation techniques involves dividing the total DoFs of the GFEM into omitted and retained DoFs such that:

$$
\mathbf{x}=\left[\begin{array}{l}
\mathbf{x}_{0} \\
\mathbf{x}_{r}
\end{array}\right]
$$

where $\mathbf{x}_{o} \in \mathbb{R}^{\left(N-n_{r}\right) \times 1}$ is the displacement vector corresponding to the omitted DoFs of the GFEM.

Assuming no loads are applied to the omitted DoFs, and by substituting Eq. (2.10) into Eq. 7, ignoring damping, results in:

$$
\left(-\omega^{2}\left[\begin{array}{ll}
\mathbf{M}_{o o} & \mathbf{M}_{o r} \\
\mathbf{M}_{r o} & \mathbf{M}_{r r}
\end{array}\right]+\left[\begin{array}{ll}
\mathbf{K}_{o o} & \mathbf{K}_{o r} \\
\mathbf{K}_{r o} & \mathbf{K}_{r r}
\end{array}\right]\right)\left[\begin{array}{l}
\mathbf{x}_{o} \\
\mathbf{x}_{r}
\end{array}\right]=\left[\begin{array}{c}
0 \\
\mathbf{f}_{r}
\end{array}\right]
$$

Using Eq. (2.11), the omitted DoFs can be expressed as a linear combination of the retained DoFs through a linear dependent operator matrix, $\Psi \in \mathbb{R}^{\left(\mathrm{N}-\mathrm{n}_{\mathrm{r}}\right) \times \mathrm{n}_{\mathrm{r}}}$, expressed as:

$$
\mathbf{x}_{o}=\Psi \mathbf{x}_{r}
$$

The total displacement vector of the GFEM can then be rewritten as, 


$$
\mathbf{x}=\left[\begin{array}{c}
\Psi \\
\mathbf{I}_{r}
\end{array}\right] \mathbf{x}_{r}=\mathbf{T} \mathbf{x}_{r}
$$

where $\mathbf{I}_{r} \in \mathbb{R}^{n_{r} \times n_{r}}$ is the identity matrix and $\mathbf{T} \in \mathbb{R}^{N \times n_{r}}$ is the transformation matrix representing the condensed subspace.

It should be noted that the retained DoFs are chosen carefully so that the overall behavior of the reduced system will be equivalent to that of the GFEM. Various studies are conducted for the proper selection of the retained DoFs for many applications [79-83].

The most common condensation methods available in the literature include the GR $[18,19]$, IRS [22] and DY [24]. Brief description of each of these methodologies is given below.

\subsection{Guyan-Irons Reduction}

Guyan-Irons reduction, commonly known as GR or static condensation, is one of the oldest and simplest MOR methods available in literature $[18,19]$. The reduced subspace for the GR is formed by ignoring the inertial contribution which reduces the system equation of motion (Eq.(2.11)) to its static formulation. Accordingly, the omitted DoFs can be expressed in terms of the retained DoFs as:

$$
\mathbf{x}_{o}=-\mathbf{K}_{o o}^{-1} \mathbf{K}_{o r} \mathbf{x}_{r}=\mathbf{\Psi}_{G R} \mathbf{x}_{r}
$$

where $\boldsymbol{\Psi}_{G R}$ is the GR linear dependent operator matrix. Thus, the total displacement vector, $\mathbf{x}$, can be written as, 


$$
\mathbf{x}=\left[\begin{array}{c}
\mathbf{x}_{o} \\
\mathbf{x}_{r}
\end{array}\right]=\left[\begin{array}{c}
\mathbf{\Psi}_{G R} \\
\mathbf{I}_{r}
\end{array}\right] \mathbf{x}_{r}=\mathbf{T}_{G R} \mathbf{x}_{r}
$$

where $\left[\mathbf{T}_{G R}\right] \in \mathbb{R}^{N \times n_{r}}$ is the transformation matrix of the GR method constructed as a concatenation of the interface constraint modes. An interface constraint mode is defined as the static deformation of the unloaded GFEM when a unit displacement is applied to a single DoF of the retained grid points while the remaining retained DoF of the system are restrained.

By post and pre- multiplying of Eq. (2.7) by the GR transformation matrix, $\mathbf{T}_{G R}$, and its transpose, respectively, results in the equation of motion of the GR ROM as:

$$
\left(-\omega^{2} \mathbf{M}_{G R}+\mathbf{K}_{G R}\right) \mathbf{x}_{r}=\mathbf{f}_{r}
$$

where $\mathbf{M}_{G R}=\mathbf{T}_{G R}^{T} \mathbf{M} \mathbf{T}_{G R}$ and $\mathbf{K}_{G R}=\mathbf{T}_{G R}^{T} \mathbf{K T}_{G R}$ are the Guyan reduced mass and stiffness matrices.

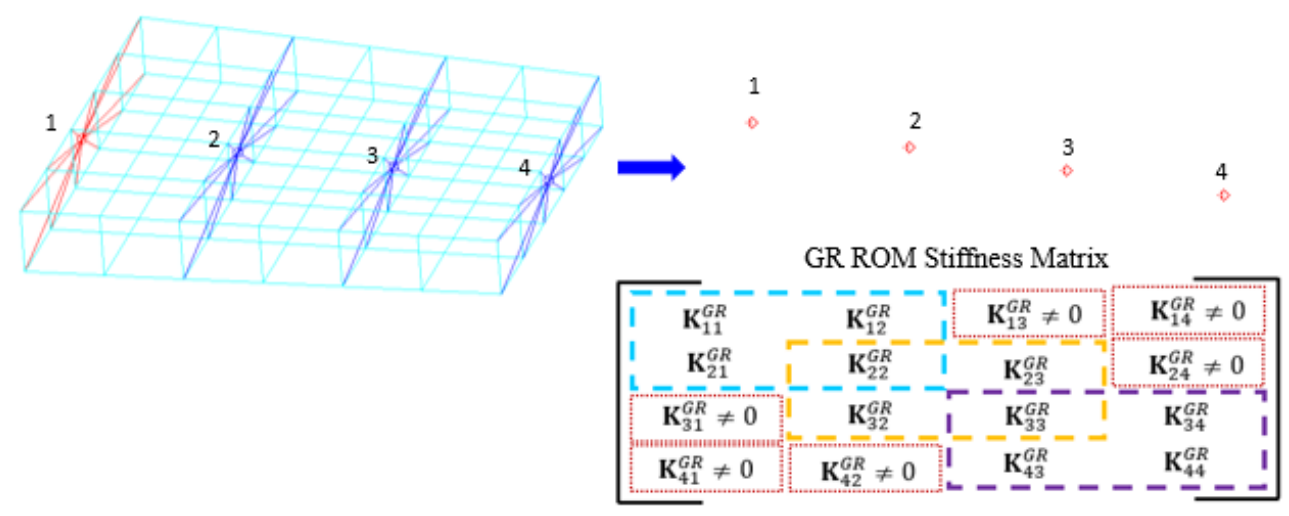

Figure 2.2 GR reduced Stiffness matrix

Figure 2.2 shows a schematic representation of the GR ROM of the same 3 bays of the wing-box GFEM. Here, the grid points representing the wing-box stations in Figure 2.1 are 
considered here as a cloud of grid points of the retained DoFs of the GFEM. Accordingly, dimensions of the stiffness matrices of the SM and direct condensation ROMs, namely, GR, IRS and DY, are the same.

\subsection{Improved Reduction system}

O'Callahan introduced another reduction methodology named as IRS where the transformation matrix of the condensed subspace is formed taking into consideration inertial effects [22]. Accordingly, by using Eq. (2.11), the omitted DoFs can be expressed in terms of retained DoFs as,

$$
\mathbf{x}_{o}=\frac{-\left(-\omega^{2} \mathbf{M}_{o r}+\mathbf{K}_{o r}\right) \mathbf{x}_{r}}{\left(\mathbf{K}_{o o}-\omega^{2} \mathbf{M}_{o o}\right)}=-\left(\mathbf{K}_{o o}-\omega^{2} \mathbf{M}_{o o}\right)^{-1}\left(-\omega^{2} \mathbf{M}_{o r}+\mathbf{K}_{o r}\right) \mathbf{x}_{r}
$$

The term $\left(\mathbf{K}_{o o}-\omega^{2} \mathbf{M}_{o o}\right)^{-1}$ can be approximated using the binomial theorem [23]. The binomial expansion series is truncated after the second order terms which results in,

$$
\mathbf{x}_{o}=\left\{-\mathbf{K}_{o o}^{-1} \mathbf{K}_{o r}+\mathbf{K}_{o o}^{-1}\left(\mathbf{M}_{o r}+\mathbf{M}_{o o}\left(-\mathbf{K}_{o o}^{-1} \mathbf{K}_{o r}\right)\right) \omega^{2}\right\} \mathbf{x}_{r}
$$

Using equation (2.16) and by considering the free response of the GR ROM, the eigenvalues of the reduced system can be expressed as,

$$
\omega^{2} \mathbf{x}_{r}=\mathbf{M}_{G R}^{-1} \mathbf{K}_{G R} \mathbf{x}_{r}
$$

Substitute Eq.(2.19) into Eq.(2.18), results in,

$$
\mathbf{x}_{o}=\left(-\mathbf{K}_{o o}^{-1} \mathbf{K}_{o r}\right) \mathbf{x}_{r}+\left(\mathbf{K}_{o o}^{-1}\left(\mathbf{M}_{o r}+\mathbf{M}_{o o}\left(-\mathbf{K}_{o o}^{-1} \mathbf{K}_{o r}\right)\right) \mathbf{M}_{G R}^{-1} \mathbf{K}_{G R}\right)\left\{\mathbf{x}_{r}\right\}
$$

Equation (2.20) can be simplified as, 


$$
\mathbf{x}_{o}=\Psi_{G R} \mathbf{x}_{r}+\Psi_{I R S} \mathbf{x}_{r}
$$

where $\boldsymbol{\Psi}_{I R S}$ is the inertial correction matrix and it is given by,

$$
\mathbf{\Psi}_{I R S}=\left(\mathbf{K}_{o o}^{-1}\left(\mathbf{M}_{o r}+\mathbf{M}_{o o}\left(-\mathbf{K}_{o o}^{-1} \mathbf{K}_{o r}\right)\right) \mathbf{M}_{G R}^{-1} \mathbf{K}_{G R}\right)
$$

Accordingly, the IRS transformation matrix is expressed as,

$$
\mathbf{T}_{I R S}=\left[\begin{array}{c}
\boldsymbol{\Psi}_{G R}+\boldsymbol{\Psi}_{I R S} \\
\mathbf{I}_{r}
\end{array}\right]
$$

The IRS reduced equation of motion can be written as,

$$
\left(-\omega^{2} \mathbf{M}_{I R S}+\mathbf{K}_{I R S}\right) \mathbf{x}_{r}=\mathbf{f}_{r}
$$

where $\mathbf{M}_{I R S}=\mathbf{T}_{I R S}^{T} \mathbf{M T}_{I R S}$ and $\mathbf{K}_{I R S}=\mathbf{T}_{I R S}^{T} \mathbf{K} \mathbf{T}_{I R S}$ are the reduced mass and stiffness matrices, respectively.

Blair, et.al. pointed out that by using an iterative procedure, the reduced order basis vectors formed by the IRS method can be improved [84]. Here, the reduced Guyan mass and stiffness matrices are substituted in Eq.(2.22) to calculate the inertial correction matrix. It is possible to update the inertial correction matrix with the $\mathbf{M}_{I R S}$ and $\mathbf{K}_{I R S}$ to form a better correction matrix, $\boldsymbol{\Psi}_{I R S}$. Thus, it can be done in an iterative scheme as shown below.

Substituting the new updated IRS reduced matrices instead of $\mathbf{K}_{G R}$ and $\mathbf{M}_{G R}$ in Eq.(2.22) gives,

$$
\boldsymbol{\Psi}_{I R S, i}=\left(\mathbf{K}_{o o}^{-1}\left(\mathbf{M}_{o r}+\mathbf{M}_{o o}\left(-\mathbf{K}_{o o}^{-1} \mathbf{K}_{o r}\right)\right) \mathbf{M}_{I R S, i-1}^{-1} \mathbf{K}_{I R S, i-1}\right)
$$


For the first iteration with i=1: $\mathbf{M}_{I R S, 0}^{-1}=\mathbf{M}_{G R}^{-1}$ and $\mathbf{K}_{I R S, 0}=\mathbf{K}_{G R}$

So the updated transformation matrix is,

$$
\mathbf{T}_{I R S, i}=\left[\begin{array}{c}
\boldsymbol{\Psi}_{G R}+\boldsymbol{\Psi}_{I R S, i} \\
\mathbf{I}_{r}
\end{array}\right]
$$

Similar to Eq.(2.24), the IRS reduced equation of motion using the updated IRS transformation $\left(\mathbf{T}_{I R S, i}\right)$ can be calculated.

\subsection{DYnamic condensation}

DY method includes the approximation of the mass effects of the omitted DoF in the reduced subspace [24]. Here, Eq. (2.7) can be written as:

$$
\mathbf{Q}\left[\begin{array}{l}
\mathbf{x}_{o} \\
\mathbf{x}_{r}
\end{array}\right]=\left[\begin{array}{ll}
\mathbf{Q}_{o o} & \mathbf{Q}_{o r} \\
\mathbf{Q}_{r o} & \mathbf{Q}_{r r}
\end{array}\right]\left[\begin{array}{c}
\mathbf{x}_{o} \\
\mathbf{x}_{r}
\end{array}\right]=\left[\begin{array}{c}
0 \\
\mathbf{f}_{r}
\end{array}\right]
$$

where $\mathbf{Q}=-\omega^{2} \mathbf{M}+\mathbf{K}$. Here, $\omega^{2}$ is an arbitrary eigenvalue supplied by the user. Compared to the GR, the DY method provides a better approximation of the reduced system response around the user supplied eigenvalue.

Similar to the previous methods, the omitted DoFs can be written as a linear combination of the retained DoFs as,

$$
\mathbf{x}_{o}=\mathbf{Q}_{o o}^{-1} \mathbf{Q}_{o r} \mathbf{x}_{r}
$$

Accordingly, the total displacement vector of the GFEM can be written as, 


$$
\mathbf{x}=\left[\begin{array}{c}
\mathbf{Q}_{o o}^{-1} \mathbf{Q}_{o r} \\
\mathbf{I}_{r}
\end{array}\right] \mathbf{x}_{r}=\mathbf{T}_{D Y} \mathbf{x}_{r}
$$

where $\mathbf{T}_{D Y}$ is the DY transformation matrix.

The DY ROM be expressed as,

$$
\left(-\omega^{2} \mathbf{M}_{D Y}+\mathbf{K}_{D Y}\right) \mathbf{x}_{r}=\mathbf{f}_{r}
$$

where $\mathbf{M}_{D Y}=\mathbf{T}_{D Y}^{T} \mathbf{M T}_{D Y}$ and $\mathbf{K}_{D Y}=\mathbf{T}_{D Y}^{T} \mathbf{K T}_{D Y}$ are, respectively, the reduced mass and stiffness matrices of the DY ROM.

It should be noted that at zero frequency, DY method retrieves a ROM identical to that of the GR. As the system frequency increases, the omitted mass neglected in the GR formulations becomes more significant $[85,59]$.

\subsubsection{Interpolatory methods}

Interpolatory methods are aimed to alleviate high computing cost associated with the frequency response analysis of large GFEM. The idea of the interpolatory method is to compute the system response at a set of sampling frequencies and the total system response is approximated by interpolating throughout the frequency range of interest. This involves the formation of the reduced subspace on which the large matrices the detailed GFEM are projected. The reduced subspace is formed using the first $j$ terms in the Taylor series expansion of the frequency response function. Commonly used interpolatory methods are DGP and KGP. DGP uses the response function and its derivatives at a sampling frequency to form the projection subspace whereas KGP rely on the formation of the Krylov subspace 
which spans the derivatives of the frequency response function. The formation of the reduced basis vectors for these methods are explained below.

\subsection{Derivative based Galerkin projection}

The basis vectors are formed by the recursive differentiation of displacement function $\mathbf{x}$ in Eq. (2.7) with respect to, the frequency, $\omega$, in the sampling frequency set $(\Delta \omega)$ [49]. Subsequently, the generated basis vectors are made orthonormal by employing a modified Gram-Schmidt algorithm [50]. These orthonormal basis vectors form the transformation matrix $\left(T_{D G P}\right)$ as shown below,

$$
\mathbf{T}_{D G P}=\overbrace{k=1}^{P} \operatorname{\oplus pan}\left\{\mathbf{x}\left(\omega_{k}\right), \frac{d \mathbf{x}\left(\omega_{k}\right)}{d \omega}, \ldots, \frac{d^{j-1} \mathbf{x}\left(\omega_{k}\right)}{d \omega^{j-1}}\right\}, \Delta \omega \in\left\{\omega_{1}, \omega_{2}, \ldots, \omega_{P}\right\}
$$

where $P$ is the number of sampling points in the frequency interval $(\Delta \omega)$.

\subsection{Krylov based Galerkin projection}

Krylov based Galerkin projection (KGP) [44] is formed using the Krylov vectors

which spans the derivatives of the frequency response function of Eq. (2.31). The reduced Krylov subspace for the dynamic system with proportional damping can be expressed as:

$$
\mathbf{T}_{K G P}=\overbrace{k=1}^{P} \overbrace{\mathcal{N}} \mathcal{K}_{j}(\mathbf{H}, \mathbf{b})=\operatorname{span}\left(\mathbf{b}, \mathbf{H} \mathbf{b}, \mathbf{H}^{2} \mathbf{b}, \ldots, \mathbf{H}^{j-l} \mathbf{b}\right)
$$

where $\mathbf{H}=-\left(-\omega_{k}^{2} \mathbf{M}+i \omega_{k} \mathbf{D}+\mathbf{K}\right)^{-1} \mathbf{M}$ and $\mathbf{b}=\left(-\omega_{k}^{2} \mathbf{M}+i \omega_{k} \mathbf{D}+\mathbf{K}\right)^{-1} \mathbf{f}$.

Similarly, the reduced subspace for the non-proportional damped system is given by, 


$$
\mathbf{T}_{K G P}=\underbrace{P}_{k=1} \mathcal{K}_{j}\left(\mathbf{H}_{1}, \mathbf{H}_{2} ; \mathbf{v}_{0}\right)=\operatorname{span}\left(\mathbf{v}_{0}, \mathbf{v}_{1}, \mathbf{v}_{2}, \ldots, \mathbf{v}_{j-1}\right)
$$

where $\mathbf{H}_{1}=-\left(-\omega_{k}{ }^{2} \mathbf{M}+i \omega_{k} \mathbf{D}+\mathbf{K}\right)^{-1}\left(2 i \omega_{k} \mathbf{M}+\mathbf{D}\right), \mathbf{H}_{2}=-\left(-\omega_{k}{ }^{2} \mathbf{M}+i \omega_{k} \mathbf{D}+\mathbf{K}\right)^{-1} \mathbf{M}$ and $\mathbf{v}_{0}=\left(-\omega_{p}^{2} \mathbf{M}+i \omega_{p} \mathbf{D}+\mathbf{K}\right)^{-1} \mathbf{f}$. Also, $\mathbf{v}_{l}=\mathbf{H}_{l} \mathbf{v}_{0}$ and $\mathbf{v}_{l}=\mathbf{H}_{l} \mathbf{v}_{l-1}+\mathbf{H}_{2} \mathbf{v}_{l-2}$ for $l \geq 2$

The efficiency of Krylov vectors for MOR of second order dynamical problem is proven superior [86]. The Krylov vectors used to form the reduced subspace is equivalent to the static modes, which means the reduced model is represented in physical coordinate. In case of non-proportional damped dynamical system, the accuracy of KGP and DGP MOR techniques is far superior [48] as compared to all other MOR techniques.

\subsubsection{Modal coordinate projection based MOR methods}

Modal decomposition methods are based on forming a reduced subspace using a set of basis vector selected from the normalized eigenvectors of the dynamical system within a frequency range of interest. They can be classified into Real or complex decomposition methods [48] based on the eigenvectors used to construct the reduced subspace.

\subsubsection{Real Modal Decomposition}

This method uses the modal superposition employing the real normal modes of the undamped system to approximate the dynamic response of the structure in modal coordinates [87]. The structural response in the physical coordinates, $\mathbf{x}$, is expressed as,

$$
\mathbf{x}=\phi_{d} \mathbf{q}_{d}
$$

where $\mathbf{q}_{d} \in \mathbb{R}^{n_{d} \times 1}$ is the generalized modal coordinates and $\boldsymbol{\phi}_{\boldsymbol{d}} \in \mathbb{R}^{N \times n_{d}}$ is the modal matrix 
formed with $n_{d}$ dominant modes within a specific frequency range of interest.

Substituting Eq. (2.34) into Eq. (2.7), results in,

$$
-\omega^{2} \mathbf{M} \boldsymbol{\phi}_{d} \mathbf{q}_{d}+\mathbf{K} \boldsymbol{\phi}_{d} \mathbf{q}_{d}=\mathbf{f}
$$

By pre-multiplying Eq. (2.35) with $\boldsymbol{\phi}_{\boldsymbol{d}}^{T}$ gives the decoupled equation of motion of the reduced system $-\omega^{2} \mathbf{M}_{\phi} \mathbf{q}_{r}+\mathbf{K}_{\phi} \mathbf{q}_{r}=\mathbf{f}_{r}$ where $\mathbf{M}_{\phi}=\boldsymbol{\phi}_{d}^{T} \mathbf{M} \boldsymbol{\phi}_{\boldsymbol{d}}, \mathbf{K}_{\phi}=\boldsymbol{\phi}_{d}^{T} \mathbf{K} \boldsymbol{\phi}_{\boldsymbol{d}}$ and $\mathbf{f}_{r}=\boldsymbol{\phi}_{\boldsymbol{d}}^{T} \mathbf{f}$ are, respectively, the reduced modal mass and stiffness matrices and force vector.

Representing the ROM of the GFEM in the modal domain is not recommended within the aerospace industry as most of the integration processes among suppliers and development groups are conducted through physical coordinate integration systems.

\subsubsection{Complex Modal Decomposition}

For non-proportional damping, a decoupled equation of motion is achieved using complex modal analysis. This involves expressing the second order ordinary differential equation with $\mathrm{N}$ DoFs into first order differential equation with $2 N$ DoFs. That is, the state space formulation which is expressed as:

$$
\dot{\mathbf{z}}(t)=\mathbf{A z}(t)+\mathbf{B f}(t)
$$

where the state vector $\mathbf{z}=\left[\begin{array}{l}\mathbf{x} \\ \dot{\mathbf{x}}\end{array}\right] \in \mathbb{R}^{2 N \times 1}$, the state matrix $\mathbf{A}=\left[\begin{array}{cc}\mathbf{0} & \mathbf{I} \\ -\mathbf{M}^{-1} \mathbf{K} & -\mathbf{M}^{-1} \mathbf{D}\end{array}\right] \in \mathbb{R}^{2 N \times 2 N}$, the input matrix $\mathbf{B}=\left[\begin{array}{c}\mathbf{0} \\ \mathbf{M}^{-1}\end{array}\right] \in \mathbb{R}^{2 N \times N}$ and the applied load vector $\mathbf{f} \in \mathbb{R}^{2 N \times 1}$. It should be noted that the state matrix, $\mathbf{A}$, is real and non-symmetric matrix. 
By considering the free response of the system and substituting $\mathbf{z}=e^{\lambda} \overline{\boldsymbol{\phi}}$ into Eq.(2.36) gives the eigenvalue problem, $\lambda \overline{\boldsymbol{\phi}}=\mathbf{A} \overline{\boldsymbol{\phi}}$ which gives $2 \mathrm{~N}$ right eigenvectors. The left eigenvectors $\overline{\boldsymbol{\Psi}}$ can be determined from $\mathbf{A}^{\mathrm{T}}$. Right and left eigenvectors are truncated based on the frequency range of interest to $\overline{\boldsymbol{\phi}}_{d} \in \mathbb{C}^{2 N \times n_{d}}$ and $\overline{\boldsymbol{\Psi}}_{d} \in \mathbb{C}^{2 N \times n_{d}}$, respectively.

Using expansion theorem, the state space vector $\mathbf{z}$ can be represented in the generalized coordinate, $\mathbf{q}_{d}$, as,

$$
\mathbf{z}=\overline{\boldsymbol{\phi}}_{d} \mathbf{q}_{d}
$$

where $\mathbf{q}_{d} \in \mathbb{C}^{n_{d} \times 1}$.

In order to obtain the reduced frequency response analysis form using CMD, Eq. (2.36) must first be converted to the frequency domain using a Fourier transformation. Substituting Eq. (2.37) into the equivalent frequency domain system of Eq. (2.36) and premultiplying the result by the truncated left eigenvectors $\overline{\boldsymbol{\Psi}}_{d}$ results in the uncoupled reduced state space matrix [48].

CMD method is computationally expensive because the dimension of the full matrices are doubled rendering this methodology inadequate for huge aerospace system applications.

\subsubsection{Proper Orthogonal Decomposition}

POD aims at the formation of reduced subspace using the Proper Orthogonal Modes (POMs). The POMs are evaluated based on a response matrix which is formed by the ensemble of response vector $(\mathbf{x})$ at different sampling frequencies $(\omega)$ within the frequency 
range of operation. There are various methods in literature to extract the POM based on the response matrix [67], but here the POM formation based on Singular Value Decomposition (SVD) is described below,

The response matrix $(\mathbf{R})$ is formed by the taking $P$ observations of the $N$ dimensional $\mathbf{x}$ vector at different sampling frequencies in $\Delta \omega$, accordingly, the response matrix, $\mathbf{R}$, can be formulated as,

$$
\mathbf{R}=\left[\begin{array}{lll}
\mathbf{x}_{1} & \cdots & \mathbf{x}_{n_{r}}
\end{array}\right]=\left[\begin{array}{ccc}
x_{11} & \cdots & x_{1 P} \\
\vdots & \cdots & \vdots \\
x_{N 1} & \cdots & x_{N P}
\end{array}\right]
$$

The real factorization of $\mathbf{R}$ is performed using SVD to obtain the POM,

$$
\mathbf{R}=\mathbf{U S V}^{\mathbf{T}}
$$

where $\mathbf{U}$ is an orthonormal matrix containing the left singular vectors, $\mathbf{S}$ is a diagonal matrix with singular values along the diagonal and $\mathbf{V}$ is an orthonormal matrix containing the right singular vectors.

Here the POMs are the left singular vectors $(\mathbf{U})$ of response matrix $\mathbf{R}$. Subsequently, high dimensional data contained in Eq. (2.7) will be projected to the subspace spanned by the POM basis vectors to form the POD ROM.

\subsubsection{Hybrid coordinate projection based MOR methods}

Transformation matrices of this technique are constructed employing a combination of physical and modal coordinate basis vectors. Hybrid coordinate projection based MOR methods, also known as CMS is a dynamic sub-structuring method [70]. CMS method 
consists of dividing the large GFEM into several substructures. Each substructure DoFs are classified into: interior coordinates $(i)$ and boundary coordinates $(b)$. Accordingly, Eq. (2.7) can be subdivided as,

$$
\left(-\omega^{2}\left[\begin{array}{ll}
\mathbf{M}_{i i} & \mathbf{M}_{i b} \\
\mathbf{M}_{b i} & \mathbf{M}_{b b}
\end{array}\right]+\left[\begin{array}{ll}
\mathbf{K}_{i i} & \mathbf{K}_{i b} \\
\mathbf{K}_{b i} & \mathbf{K}_{b b}
\end{array}\right]\right)\left[\begin{array}{l}
\mathbf{x}_{i} \\
\mathbf{x}_{b}
\end{array}\right]=\left[\begin{array}{c}
\mathbf{0} \\
\mathbf{f}_{b}
\end{array}\right]
$$

The physical displacement vector of the interior DoFs, $\mathbf{x}_{i}$, is expressed as a function of the generalized coordinates $\mathbf{q}_{i}$, of the interior DoFs. The total displacement vector of the GFEM can be expressed using Ritz coordinate transformation technique [87] as,

$$
\mathbf{x}=\mathbf{T}_{C M S}\left[\begin{array}{c}
\mathbf{q}_{i} \\
\mathbf{x}_{b}
\end{array}\right]
$$

where $\mathbf{T}_{C M S}$ is a transformation matrix formed by the concatenation of two or more component modes such as normal, constraint, attachment and rigid body modes. $\mathbf{q}_{i} \in$ $\mathbb{R}^{n_{i} \times 1}$ and $\mathbf{x}_{b} \in \mathbb{R}^{n_{b} \times 1}$. Here, $n_{i}=N-n_{b}$.

The two common CMS superelements methodologies discussed include, the Craig Bampton $(\mathrm{CB})$ and free boundary (FF) reduction methods. A brief description of these two methodologies is given below.

\subsubsection{Fixed Interface method}

Most common fixed interface method is the CB method which uses a combination of fixed interface normal modes and interface constraint modes for the formation of the transformation matrix [25]. The $\mathrm{CB}$ reduced displacement vector can be written in terms of dominant generalized coordinates $\mathbf{q}_{d}$ and physical boundary coordinates $\mathbf{x}_{b}$ as 


$$
\mathbf{x}=\left[\begin{array}{ll}
\boldsymbol{\phi}_{i-d} & \mathbf{T}_{G R}
\end{array}\right]\left[\begin{array}{c}
\mathbf{q}_{i-d} \\
\mathbf{x}_{b}
\end{array}\right]=\left[\begin{array}{cc}
\boldsymbol{\phi}_{i i-d} & \boldsymbol{\Psi}_{G R} \\
\mathbf{0} & \mathbf{I}_{b}
\end{array}\right]\left[\begin{array}{c}
\mathbf{q}_{i-d} \\
\mathbf{x}_{b}
\end{array}\right]
$$

where $\mathbf{T}_{C B}=\left[\begin{array}{ll}\boldsymbol{\phi}_{i-d} & \mathbf{T}_{G R}\end{array}\right] \in \mathbb{R}^{N \times n_{r}}$ is the CB transformation matrix. Here, the number of boundary DoFs $n_{b}$ are equal to the retained DoFs $n_{r}$ in the GR. Hence the constraint mode matrix considered for both $\mathrm{CB}$ and FF methods are same as the GR transformation matrix $\mathbf{T}_{G R}$. Also, $\mathbf{q}_{i-d} \in \mathbb{R}^{n_{d} \times 1}$ is the generalized coordinate vector corresponding to dominant fixed interface normal modes $\boldsymbol{\phi}_{i-d}=\left[\begin{array}{c}\boldsymbol{\phi}_{i i-d} \\ \mathbf{0}\end{array}\right] \in \mathbb{R}^{N \times n_{d}}$. The dominant fixed interface normal modes are formed by constraining all the boundary dofs $\left\{x_{b}\right\}$ and solving the eigenvalue problem of the form,

$$
\left[\mathbf{K}_{i i}-\omega_{i i}^{2} \mathbf{M}_{i i}\right] \boldsymbol{\phi}_{i i-d}=0
$$

Therefore the retained DoFs are given by,

$$
\mathbf{x}_{r}=\left[\begin{array}{c}
\mathbf{q}_{i-d} \\
\mathbf{x}_{b}
\end{array}\right]
$$

where $\mathbf{x}_{r} \in \mathbb{R}^{n_{r} \times 1}, n_{r}=n_{d}+n_{b}$.

The CB ROM can be expressed as,

$$
\left(-\omega^{2} \mathbf{M}_{C B}+\mathbf{K}_{C B}\right) \mathbf{x}_{r}=\mathbf{f}_{r}
$$

where reduced CB matrices $\mathbf{M}_{C B}$ and $\mathbf{K}_{C B}$ are given by,

$$
\mathbf{M}_{C B}=\mathbf{T}_{C B}^{T} \mathbf{M T}_{C B}, \text { where } \mathbf{M}_{C B}=\left[\begin{array}{cc}
\mathbf{I} & \left(\mathbf{M}_{i b}\right)_{d} \\
\left(\mathbf{M}_{b i}\right)_{d} & \mathbf{M}_{G R}
\end{array}\right]
$$




$$
\mathbf{K}_{C B}=\mathbf{T}_{C B}^{T} \mathbf{K T}_{C B} \text {, where } \mathbf{K}_{C B}=\left[\begin{array}{cc}
(\boldsymbol{\Lambda})_{d} & \mathbf{0} \\
\mathbf{0} & \mathbf{K}_{G R}
\end{array}\right]
$$

In Eq. (2.46), $(\boldsymbol{\Lambda})_{d}=\operatorname{diag}\left(\omega_{i i-d}^{2}\right)$, is the diagonal matrix having the dominant eigenvalues $\left(\omega_{i i-d}^{2}\right)$ of the interior dofs along the diagonals. Both $\mathbf{M}_{G R}$ and $\mathbf{K}_{G R}$ are in physical coordinate whereas remaining portion of $\mathrm{CB}$ reduced mass and stiffness matrix are in modal coordinates. In case of Test analysis models, it is necessary to transform the $\mathrm{CB}$ reduced mass and stiffness matrix portion in modal coordinates to physical coordinates. This can be done using transformation matrix given in [89].

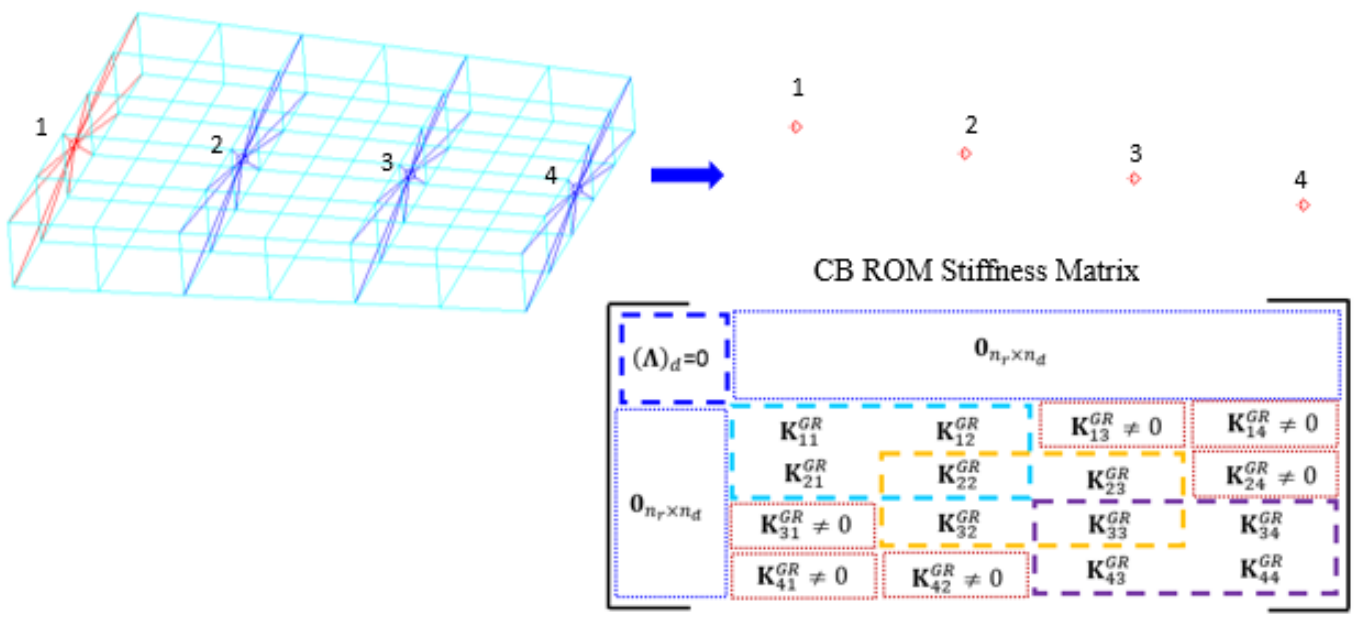

Figure 2.3 CB reduced stiffness matrix

The schematic representation of the CB ROM stiffness matrix in Eq. (46) is shown in Figure 2.3. Compared to the stiffness matrix of the SM ROM as shown in Figure 2.1, stiffness matrices of the GR and CB ROMs ,represented by Figure 2.2 and Figure 2.3 respectively, are dense with the off diagonal stiffness terms indicated by red dotted boxes which include the coupling of the DoFs ignored by the SM. For instance, entry $\mathrm{K}_{14}^{C B}$ representing the coupling between DoFs of nodes 1 and 4 are ignored by the SM 
representation while the GR and $\mathrm{CB}$ ROM are accounting for this coupling. These couplings are essential to represent the complex dynamic behavior of the GFEM.

\subsubsection{Free Boundary CMS method}

This CMS method employ free-free normal modes for the formation of transformation matrix along with static modes. CMS method [34, 95 and 96] employing only free-free normal modes produces unacceptable errors [97]. So it is a standard practice to include static modes like static interface constraint modes or attachment modes $[26,28,30]$ to make the reduced system statically complete [87]. The method followed here is based on superelements methodology [104] which uses free-free normal modes along with interface constraint modes to form the reduced subspace. The only difference as compared to $\mathrm{CB}$ method is that boundary dof is free to vibrate during the component normal mode solution. The FF CMS reduced displacement vector can be written as,

$$
\mathbf{x}_{\boldsymbol{r}}=\left[\begin{array}{ll}
\boldsymbol{\Phi}_{f-d} & \mathbf{T}_{G R}
\end{array}\right]\left[\begin{array}{c}
\mathbf{q}_{f-d} \\
\mathbf{x}_{b}
\end{array}\right]=\left[\begin{array}{cc}
\boldsymbol{\Phi}_{f_{i i-d}} & \boldsymbol{\Psi}_{i b} \\
\boldsymbol{\Phi}_{f_{b i-d}} & \mathbf{I}_{b}
\end{array}\right]\left[\begin{array}{c}
\mathbf{q}_{f-d} \\
\mathbf{x}_{b}
\end{array}\right]
$$

where $\mathbf{T}_{F F}=\left[\begin{array}{ll}\boldsymbol{\Phi}_{f-d} & \mathbf{T}_{G R}\end{array}\right] \in \mathbb{R}^{N \times n_{r}}$ is the FF transformation matrix containing dominant free interface normal modes $\boldsymbol{\phi}_{f-d} \in \mathbb{R}^{N \times n_{d}}$ and interface constraint modes $\mathbf{T}_{G R}$. The free flexible interface normal modes are generated without constraining the boundary dofs $\left\{x_{b}\right\}$, that is , $\left[\mathbf{K}-\omega_{f}^{2} \mathbf{M}\right] \boldsymbol{\Phi}_{f-d}=0$.

Therefore, the FF ROM can be expressed as,

$$
\left(-\omega^{2} \mathbf{M}_{F F}+\mathbf{K}_{F F}\right) \mathbf{x}_{r}=\mathbf{f}_{r}
$$


where $\mathbf{M}_{F F}=\mathbf{T}_{F F}^{T} \mathbf{M T}_{F F}$ and $\mathbf{K}_{F F}=\mathbf{T}_{F F}^{T} \mathbf{K T}_{F F}$ is the reduced mass matrix and stiffness matrix respectively.

The selection of dominant modes which need to be included in the CMS reduced model is a key issue for many applications [77, 90 - 93]. 


\section{Dynamic performance of Model order reduction Methodologies: a Case Study}

In this chapter, a case study is presented where the MOR methodologies discussed in chapter 2 are employed in the static and dynamic aeroelasticity analysis of a Bombardier aircraft platform. Prior to the reduction, a set of retained grid points are created along the elastic axis of the GFEM [98]. These grid points are connected to the surrounding airframe structure employing a multi-point constraints (MPC) elements to average the overall displacement of the GFEM at a specific airframe station.

In the reduction process, the GFEM with 163242 DoFs is reduced to a SM having 1290 DoFs as shown in Figure 3.1. ROMs based on linear algebraic matrix reduction methodologies is generated, with 1290 DOFs for ROM based on condensation method and 1490 DoFs for the CMS method. It should be noted that, in CMS method, the number of physical DoFs $n_{b}=1290$ which is the same as the total retained DoFs in Guyan ROM, on the other hand, as we selected only the first 200 dominant modes corresponding to the interior DoFs to be the part of the reduced structure, hence, the number of interior DoFs, $n_{d}=200$, presented in the modal coordinate. Accordingly, the total DoFs in CB ROM is $n_{r}=n_{d}+n_{b}=1490$. These additional DoFs will be kept as scalar points [100] for the static and dynamic analysis in MSC NASTRAN without increasing the number of retained grid points. 


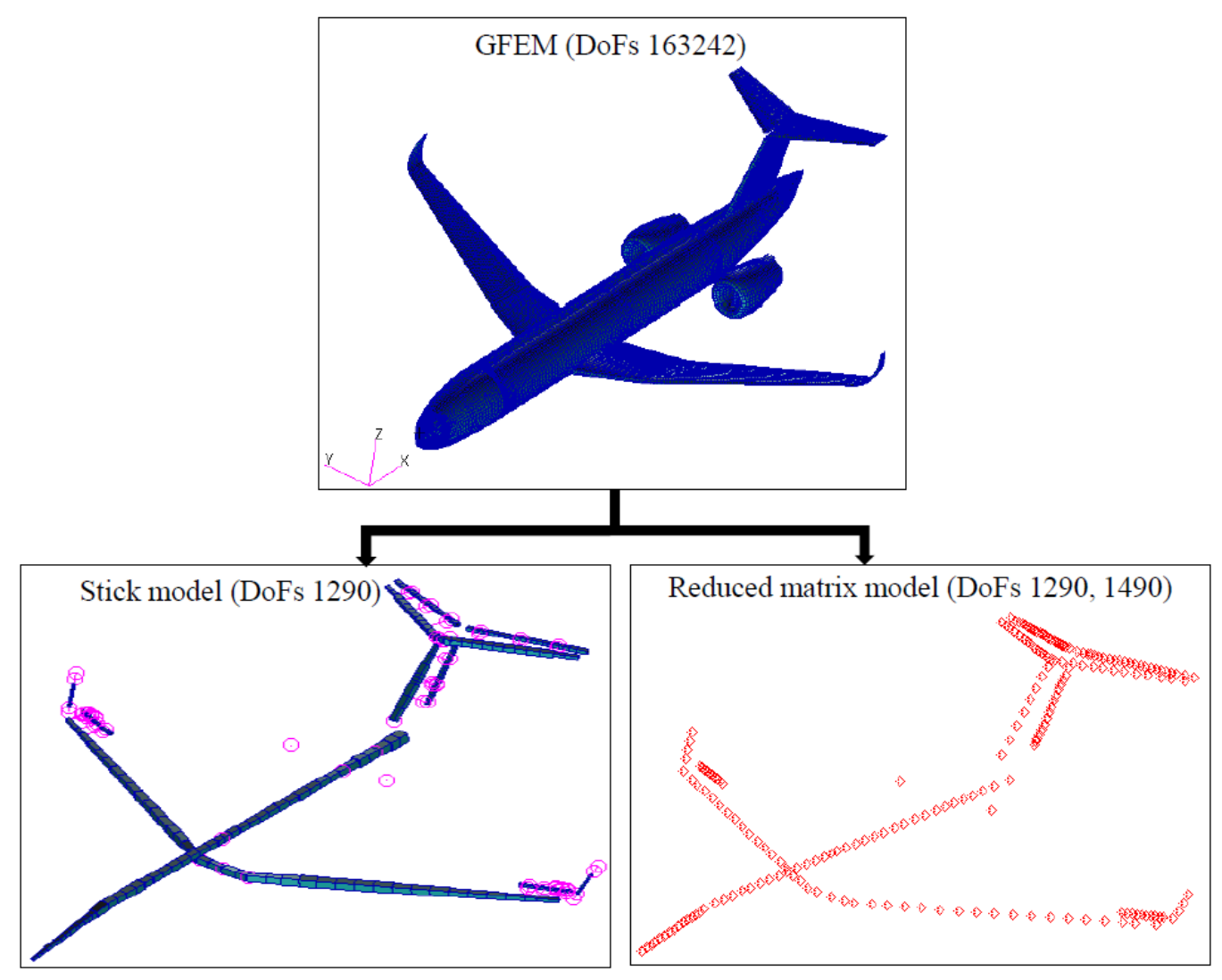

Figure 3.1 Visual representation of a generic aircraft ROMs (SM and Matrix based ROMs)

As it is a prime requirement in the aerospace industry is to develop ROMs in physical coordinate representation, hence we employ only six reduction methodologies in this case study. Modal decomposition methods are excluded as their corresponding ROMs are represented fully in the modal domain. Interpolatory methods and POD, on the other hand, are deemed computationally inefficient as their transformation matrices are dependent on the applied loads vector which requires including the MOR algorithm as part of the aeroelastic iteration process. This load dependent transformation matrix based MOR methods can be very time consuming because the ROM needs to be regenerated for all the different load cases in aeroelasticity load analysis even though the mass and stiffness case remains the same. 
As part of the current case study, aeroelasticity analysis require following inputs as shown in Figure 3.2. Apart from the airframe ROM, which forms the core part of the thesis, other inputs for the aeroelasticity analysis includes aerodynamic model, lumped mass model and the external excitation such as gust.

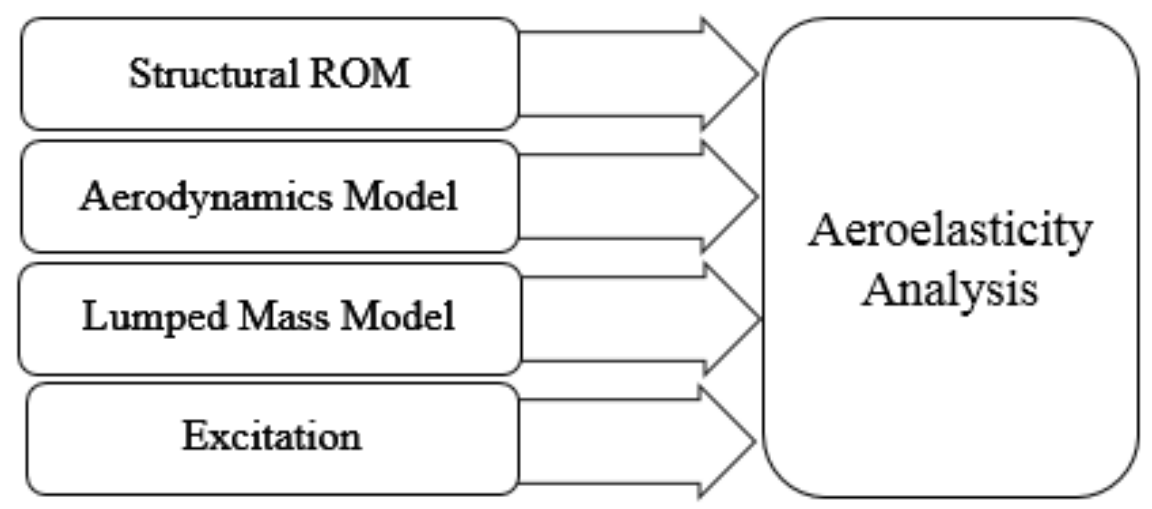

Figure 3.2 Input for aeroelasticity analysis

\section{a. Aerodynamic Model}

An aerodynamic model in aeroelasticity loads analysis is used to simulate the pressure distribution around the aircraft OML during flight. Here, an aerodynamic model based on DLM is used [105]. DLM is a potential flow-based panel method which is used to solve for unsteady aerodynamic flow across a lifting surface. The DLM is a panel method, which means that the surface is typically divided into small trapezoidal panels for computational purposes with a constant pressure distribution assumed on each panel. The aerodynamic forces is then coupled to the structural ROM retained grid points through splines [105] as shown in Figure 3.3. 


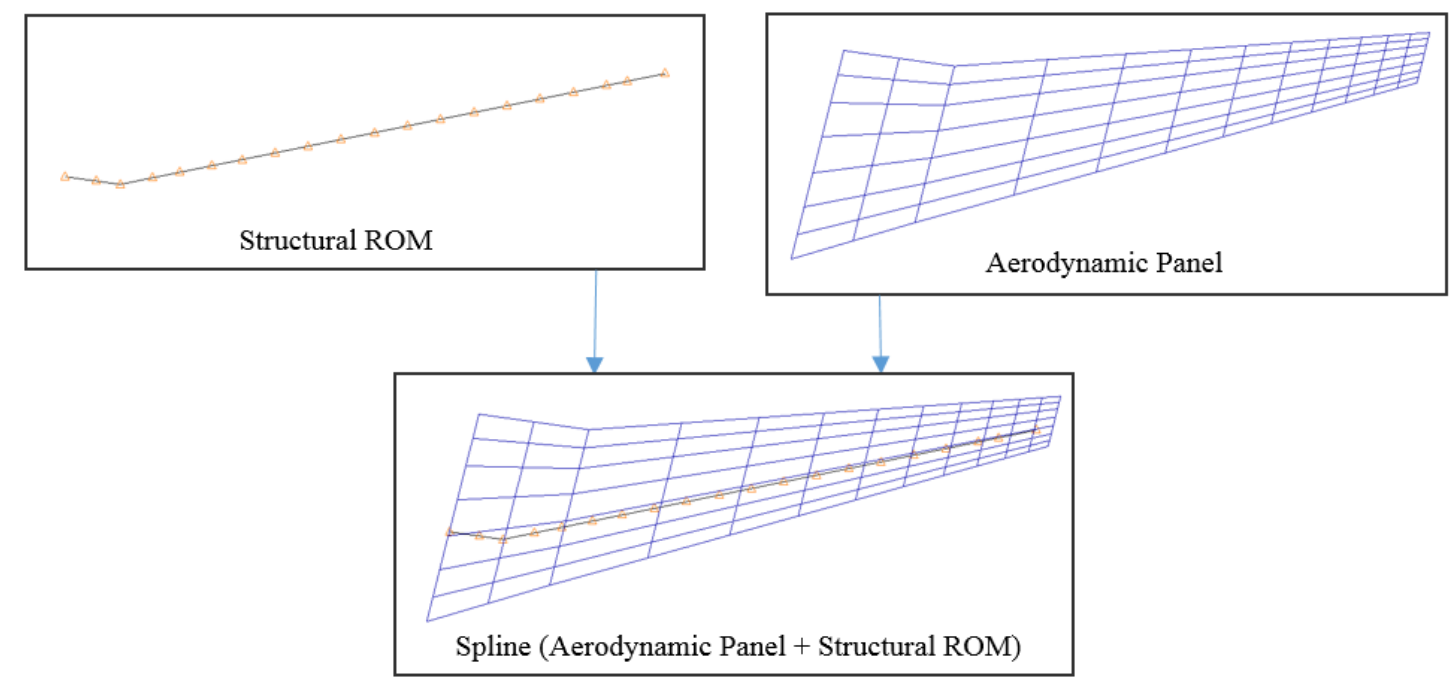

Figure 3.3 Aerodynamic DoFs coupled to the SM structural DoFs in aeroelasticity analysis

\section{b. Lumped mass model}

The standard practice in the aerospace industry for aeroelasticity analysis involves the use of lumped mass idealization of the GFEM [34]. The equivalent lumped mass [5] for each aircraft bay can be easily calculated from the aircraft CAD model. This is done by slicing each aircraft bay using a cutting plane perpendicular to the elastic axis [107]. Mass and inertia values corresponding to the sliced structure, system and payload are summed up to calculate the total mass and inertia values for each aircraft bay section. Then the concentrated mass is represented in MSC NASTRAN using concentrated mass element or CONM2 [100]. The concentrated mass representation of the 5 bays of LHS wing-box is shown in Figure 3.4. 


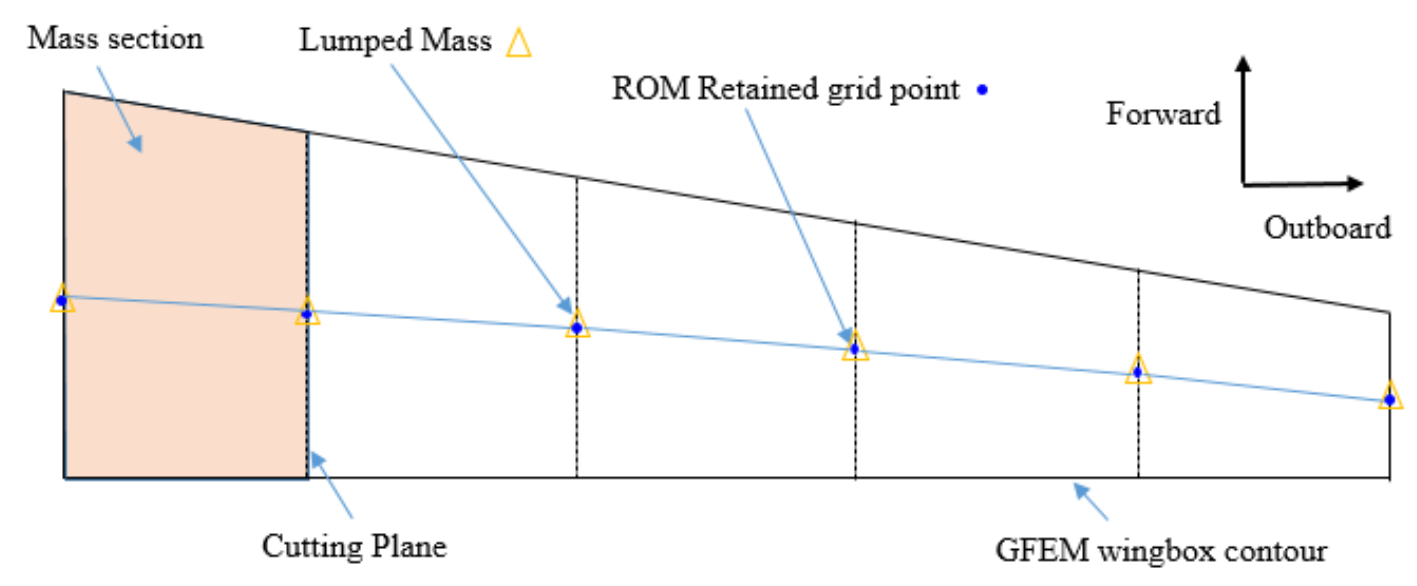

Figure 3.4 Lumped mass idealization for aircraft RHS wing-box

\section{c. Gust excitation}

There are two types of gust loading widely employed in the aircraft dynamic aeroelasticity analysis, namely, discrete gust and continuous gust $[3,4,1,5]$.

\section{Discrete Gust:}

For discrete gust load, the atmospheric disturbance is assumed to have one minus cosine velocity profile which is described as a function of time. The variation of velocity of air is normal to the flight path as shown in Eq. (3.1) and the governing equation for the variation of gust velocity $\left(V_{g}\right)$ is given by,

$$
V_{g}\left(u_{g}\right)=\frac{V_{g 0}}{2}\left(1-\cos \left(\frac{2 \pi u_{g}}{L_{g}}\right)\right), 0 \leq u_{g} \leq L_{g}
$$

where $u_{g}$ is the position of aircraft in the spatial representation of the gust with reference to a fixed location, $V_{g 0}$ is the maximum peak value of the gust, $L_{g}$ is the length of the gust. 


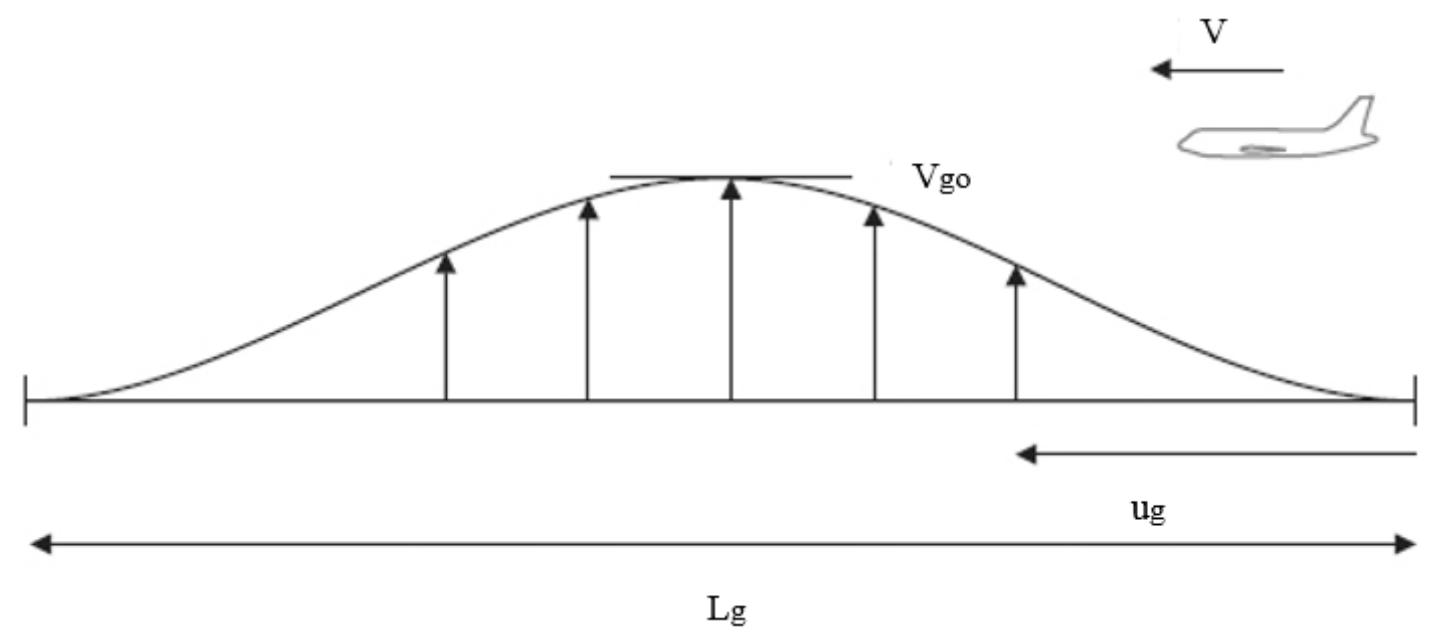

Figure 3.5 One minus cosine gust profile

\section{Continuous gust:}

For continuous gust loads, the atmospheric turbulence is assumed to have a Gaussian distribution of gust velocity intensities that can be specified in the frequency domain as a power spectral density function. According to Von Karman, the random variation of the air normal to the flight path is given by,

$$
\Phi_{g}(\Omega)=\sigma_{g}^{2} \frac{L_{t}}{\pi} \frac{1+\left(\frac{8}{3}\right)(1.339 \Omega L)^{2}}{\left[1+(1.339 \Omega L)^{2}\right]^{11 / 6}}
$$

where $\Phi_{g}(\Omega)$ denotes the random air velocity as a function of scaled frequency, $\sigma_{g}$ is the Root Mean Square (RMS) turbulence velocity, $V$ is the flight speed. $L_{t}$ is the characteristic scale wavelength of the turbulence.

It should be noted that the loads extracted in static and dynamic aeroelasticity loads analyses are normalized using the maximum load out of all the ROMs and GFEM. The 
generated ROMs are compared against the GFEM in terms of their modal pairs, static and dynamic aeroelasticity loads and the MPFs as shown below.

\subsection{Normal Mode analysis}

A normal modes analysis is performed in MSC NASTRAN to compare the reduced models natural frequencies with those of the GFEM. A comparison of the percentage error of the natural frequency corresponding to the first 10 flexible modes of the ROMs are shown in Figure 3.6. The percentage error, e, is calculated as:

$$
\mathrm{e}=\left|\frac{\omega_{i}-\omega_{G F E M}}{\omega_{G F E M}}\right| \times 100
$$

where $\omega_{i}$ and $\omega_{G F E M}$ are the natural frequency of a ROM and the GFEM, respectively.

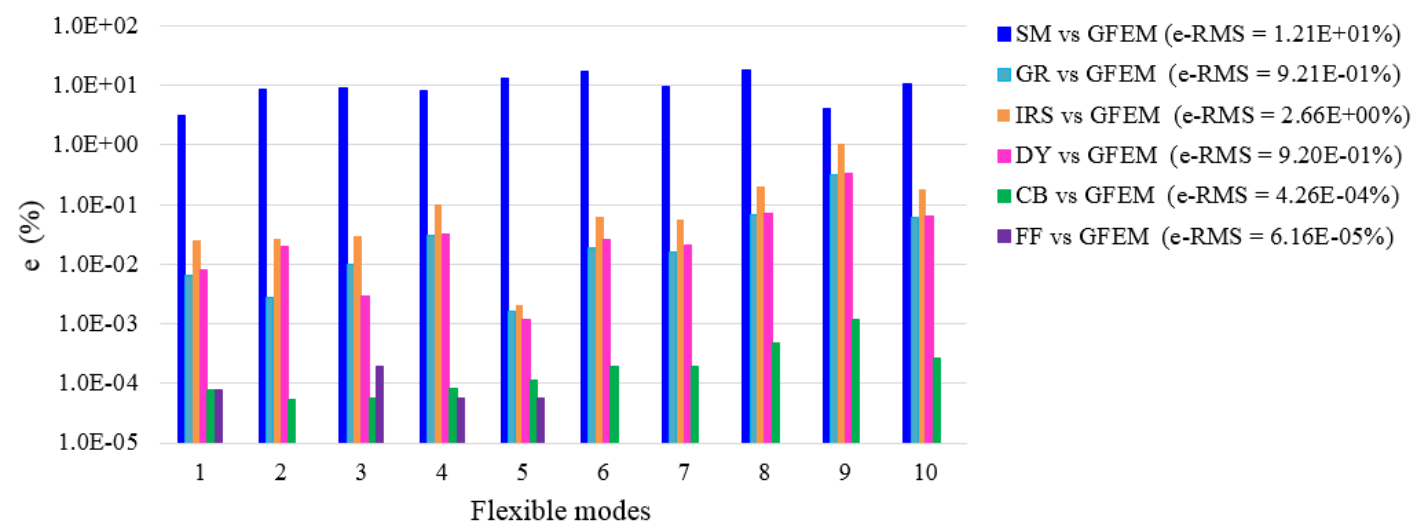

Figure 3.6 Percentage of error involved in the reduced models as compared to GFEM for the first 10 flexible natural frequencies

It can be observed from Figure 3.6 that the least Root Mean Square error (e-RMS) is associated with the FF (e-RMS $=6.16 \mathrm{E}-05)$ followed by $\mathrm{CB}(\mathrm{e}-\mathrm{RMS}=4.26 \mathrm{E}-04)$ and condensation methods such as DY, GR and IRS. The maximum error is found in the SM with e-RMS value of $12.11 \%$. 


\subsection{Static aeroelasticity load analysis}

A static aeroelasticity analysis is done to recover the static loads along the elastic axis grid points. Static aeroelastic load case of $1 \mathrm{G}$ trim condition is selected for the analysis. The trim conditions include a dynamic pressure $=14813.5 \mathrm{~Pa}$ and Mach number of 0.83 . The loads recovery for a retained grid point in wing is done by the summation of all retained grid points lying to the left side. For example, the load at grid point ID 1016 is the summed up loads from grid point IDs 1105, 1104, 1103, 1102, 1019, 1018 and 1017 as shown in Figure 3.7. This summation can be performed using monitor points method [9] in MSC NASTRAN by taking into consideration both aerodynamic and flexible loads. The out of plane shear force and the out of plane bending moment on the aircraft wing is plotted in Figure 3.8 and Figure 3.9 respectively.
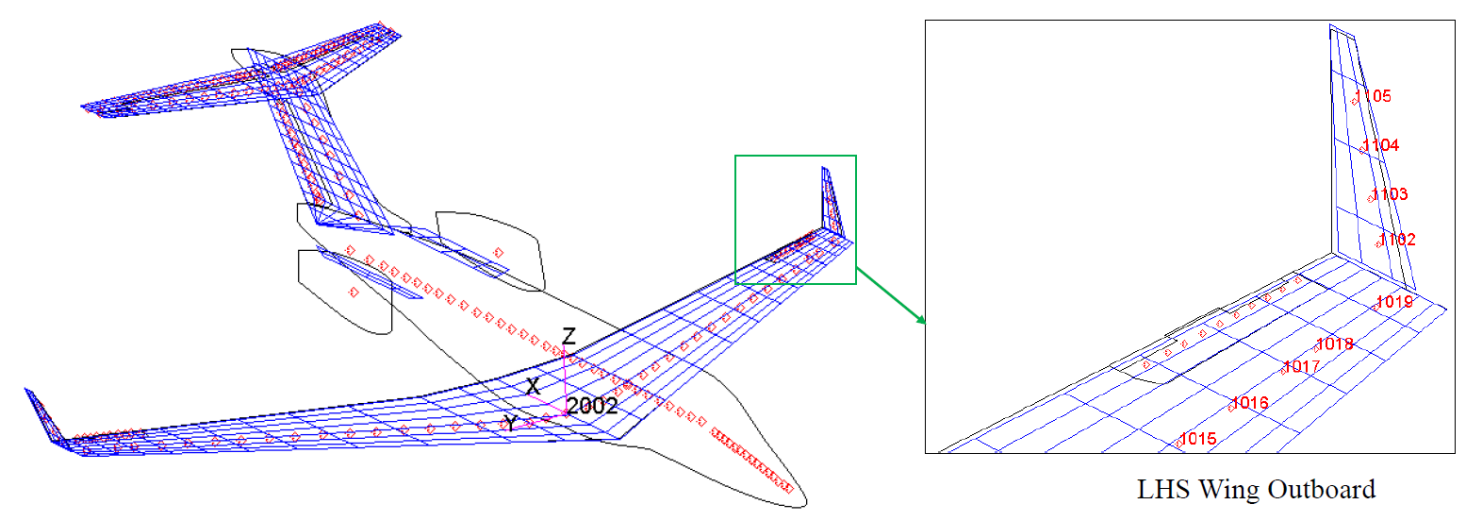

LHS Wing Outboard

Figure 3.7 Static Aeroelasticity loads recovery highlighting grid points and reference coordinate 


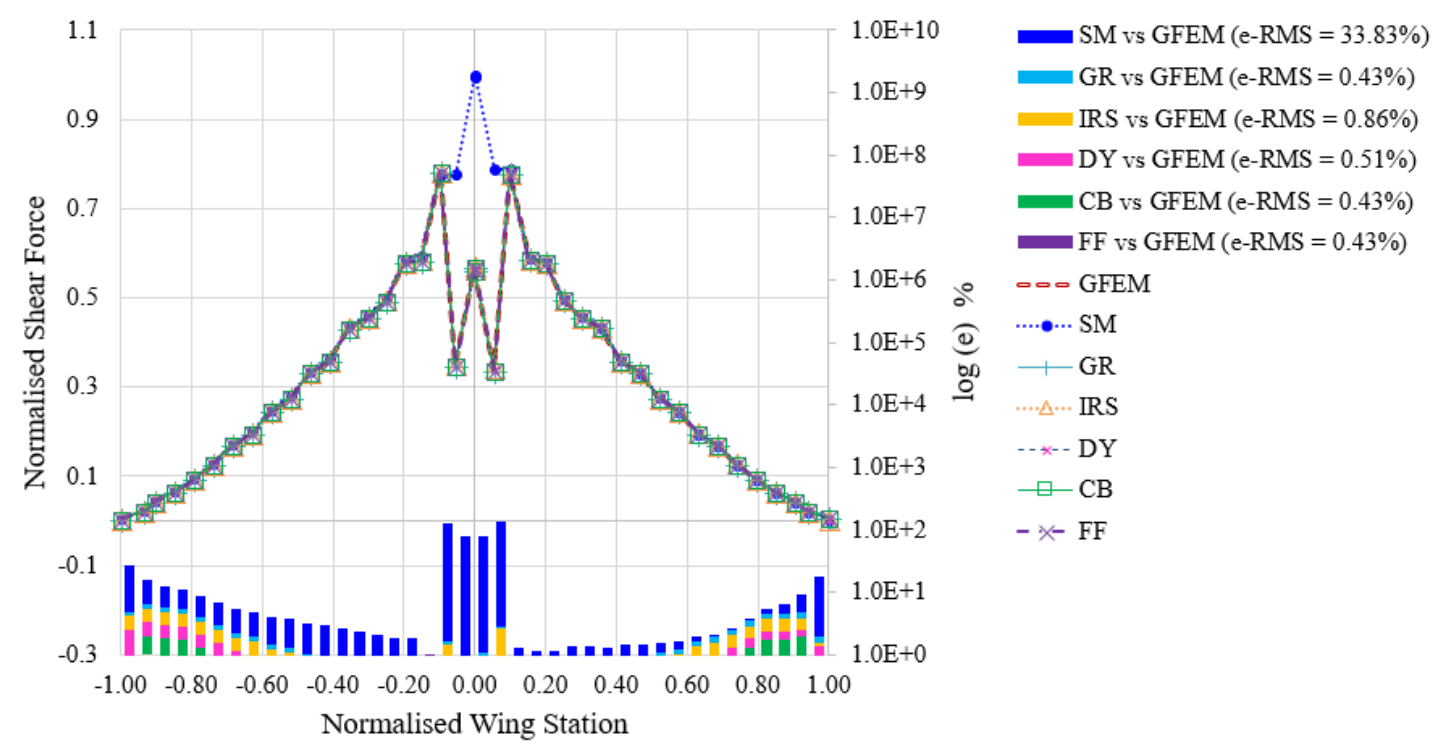

Figure 3.8 Comparison of static out of plane shear loads recovered from all ROMs and GFEM

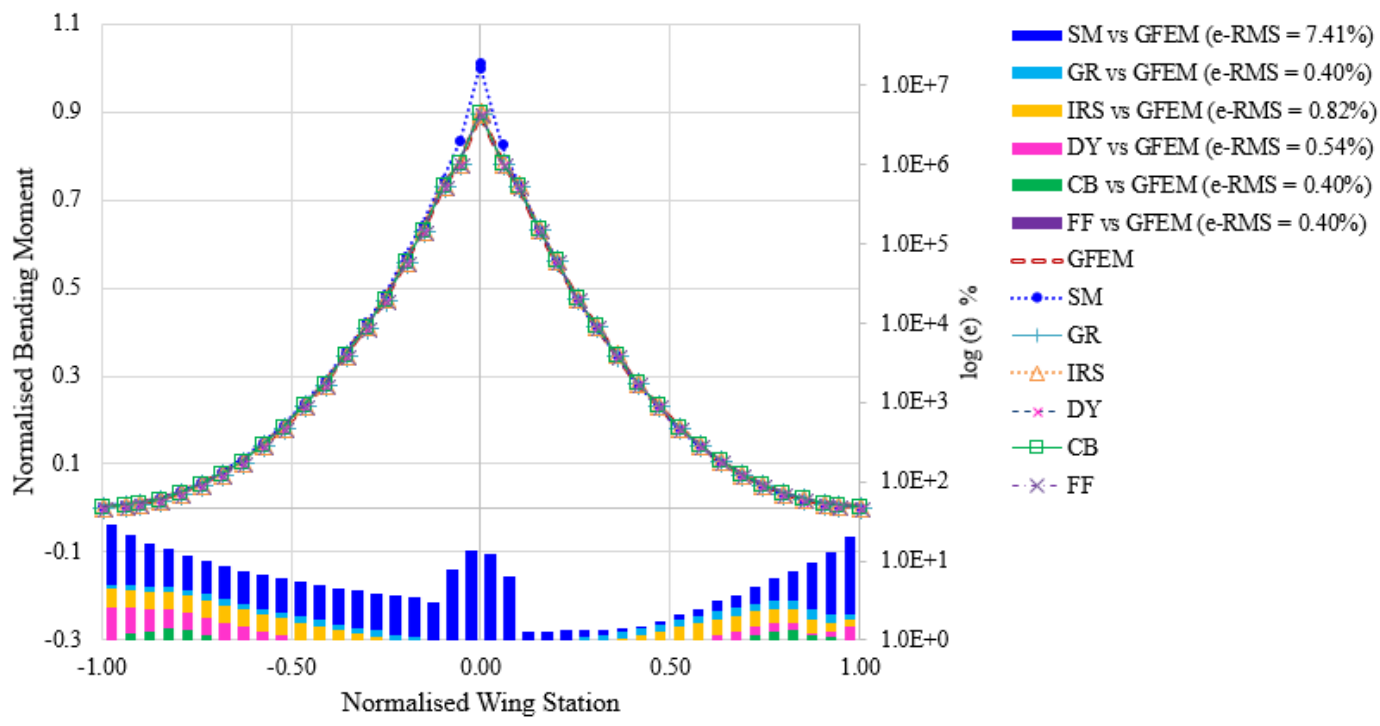

Figure 3.9 Comparison of static out-plane bending moment recovered from all ROMs and GFEM

The percentage of error associated with the static aeroelasticity loads extracted for each ROM with reference to GFEM loads is also shown in Figure 3.8 and Figure 3.9 as a stacked bar chart. The maximum error is seen in SM followed by IRS and DY ROM. On the other hand, ROMs like CB, FF and GR have the lowest percentage of error value for all the wing 
station considered. In other words, ROMs like CB, FF and GR have very good agreement with the GFEM in terms of the static aeroelastic loads recovered.

\subsection{Dynamic aeroelasticity load analysis}

A dynamic aeroelasticity analysis is performed to check the accuracy of the dynamic loads recovered from the reduced models as compared to the GFEM for two load cases, namely, TDG and Von-Karman PSD [105]. The dynamic loads are recovered from a single retained grid point ID 1019, which is located close to the tip of the left wing, with respect to the coordinate ID 1 as shown in Figure 3.10. A modal damping as a function of frequency is assumed for the analysis.

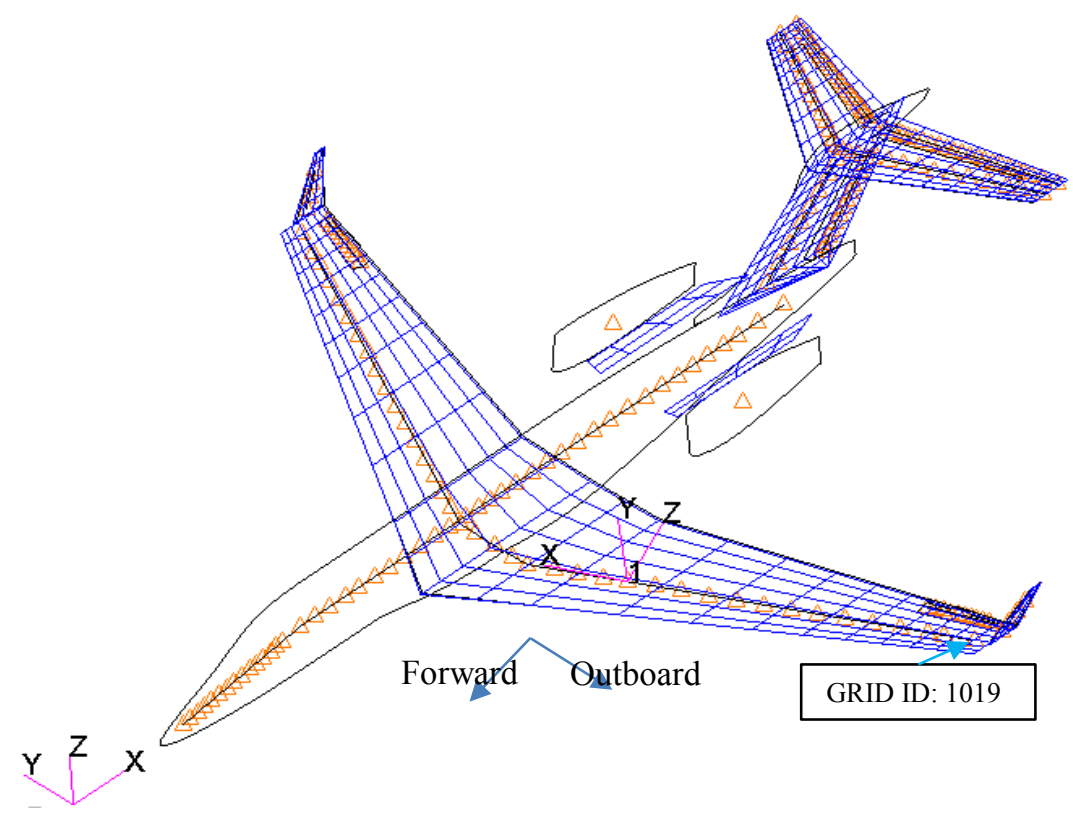

Figure 3.10 Reduced model dynamic loads recovery grid point and coordinate 


\subsubsection{Recovered dynamic load in TDG gust case}

Dynamic aeroelasticity analysis is performed to measure the response of all the ROMs to a TDG case with flight conditions shown in Table 3.1 .

Table 3.1 Flight conditions for TDG analysis

\begin{tabular}{cc}
\hline Flight conditions & Values \\
\hline Altitude & $8991.60 \mathrm{~m}$ \\
True Air speed & $252.18 \mathrm{~m} / \mathrm{s}$ \\
Equivalent Air speed (EAS) & $155.67 \mathrm{~m} / \mathrm{s}$ \\
Dynamic pressure & $14813.50 \mathrm{~Pa}$ \\
Mach Number & 0.83 \\
& $11.14 \mathrm{~m} / \mathrm{s}$ \\
Gust velocity & EAS \\
\hline
\end{tabular}

The out of plane bending moment recovered from grid point ID 1019 is shown in Figure 3.11. It can be observed from Figure 3.11 that the dynamic loads extracted from CMS ROMs conform very well with the GFEM loads with a small RMS error of $1.17 \%$ and $1.14 \%$ for CB and FF ROM respectively. Whereas the GR, IRS and DY ROMs have RMS errors of $24.92 \%, 120.78 \%$ and $22.81 \%$ respectively. The moment recovered from SM is significantly deviated from that of the GFEM with e-RMS of $345.35 \%$. 


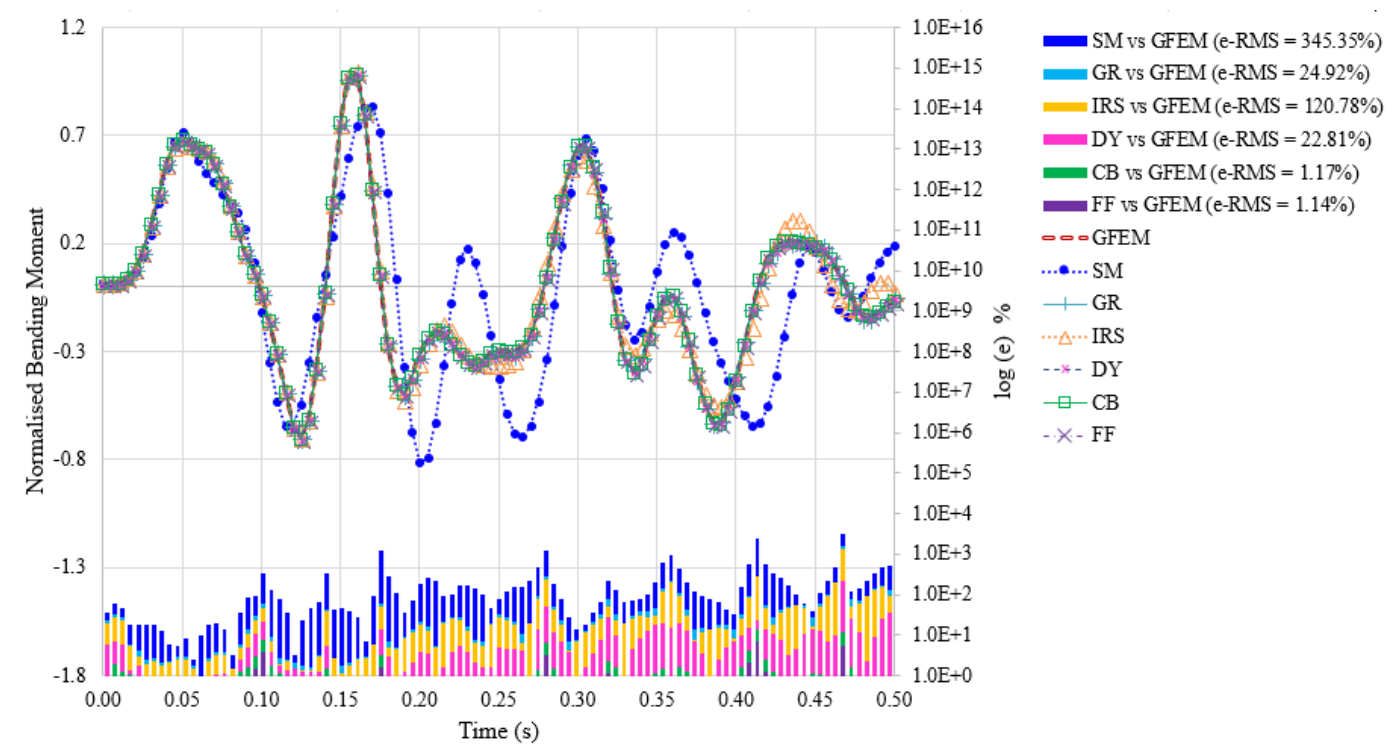

Figure 3.11 Comparison of out of plane bending moment recovered from all six ROMs and GFEM for TDG case

\subsubsection{Recovered dynamic load in PSD vertical gust case}

Similar to TDG case, dynamic aeroelasticity analysis using Von Karman PSD case is performed using the flight conditions and gust properties as shown in Table 3.2 and Table 3.3 respectively.

Table 3.2 Flight conditions for PSD gust analysis

\begin{tabular}{ll}
\hline Flight Conditions & Values \\
\hline Altitude & $0 \mathrm{ft}$ \\
True Air speed & $154.33 \mathrm{~m} / \mathrm{s}$ \\
Dynamic pressure & $14537.9 \mathrm{~Pa}$ \\
Mach Number & 0.45 \\
\hline
\end{tabular}

Table 3.3 Properties of Von Karman gust

\begin{tabular}{ll}
\hline Gust properties & Values \\
\hline Scale of gust & $762 \mathrm{~m}$ \\
RMS gust velocity & $1.0 \mathrm{~m} / \mathrm{s}$ \\
\hline
\end{tabular}


The frequency response function (FRF) magnitude and phase plots in the out of plane shear direction are shown in Figure 3.12 and Figure 3.13 respectively. The out of plane shear load recovered from the PSD gust analysis is represented in FRF of the structural response $[9,106]$. It should be noted that the FRF relates the PSD output function $\left(\Phi_{o}\right)$ and the input Von Karman signal $\left(\Phi_{i}\right)$ by,

$$
\Phi_{o}(\omega)=F R F^{2} \times \Phi_{i}(\omega)
$$

It can be seen from these plots that the SM behavior is clearly not in agreement with the reference GFEM FRF with large RMS error of $54.52 \%$ and $797.20 \%$ for magnitude and phase plots respectively. Whereas, the dynamic loads extracted from the matrix based ROMs shows a good agreement with those of GFEM. CB and FF ROMs overlap GFEM solution almost everywhere in the specific frequency range of interest. On the other hand, condensation methods like GR, IRS and DY capture the max loads recovered from GFEM but their behavior starts to deviate from GFEM at higher frequencies.

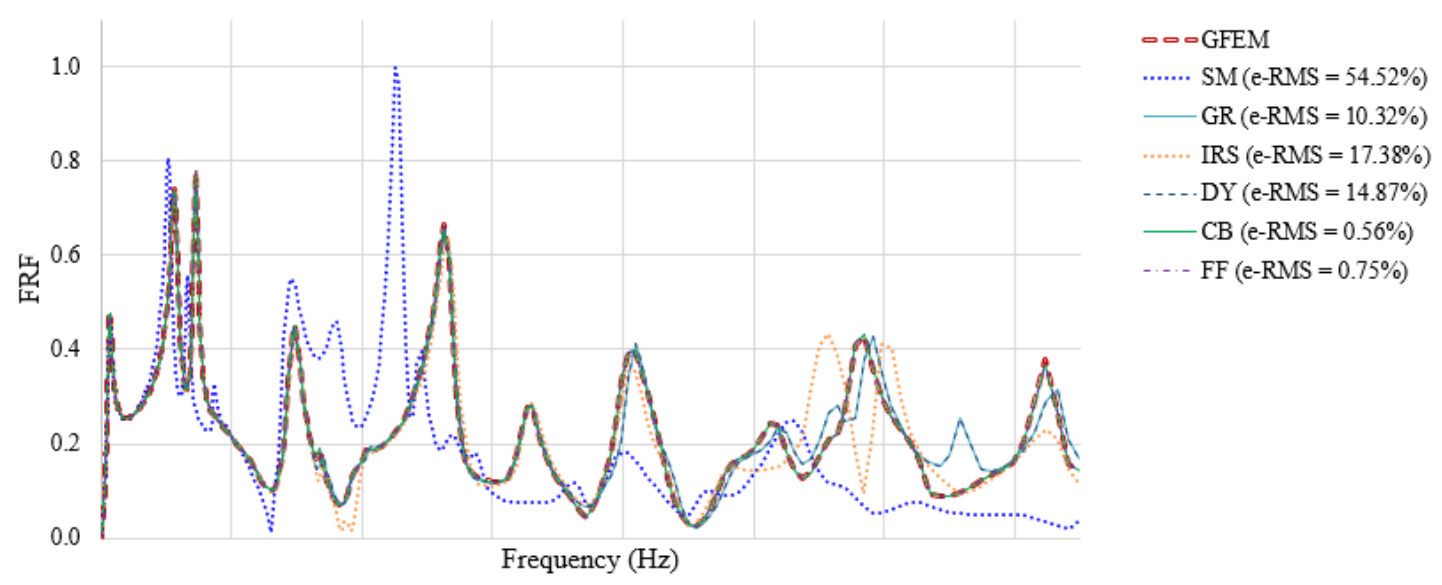

Figure 3.12 Comparison frequency response function (Magnitude) for all six ROMs and GFEM 


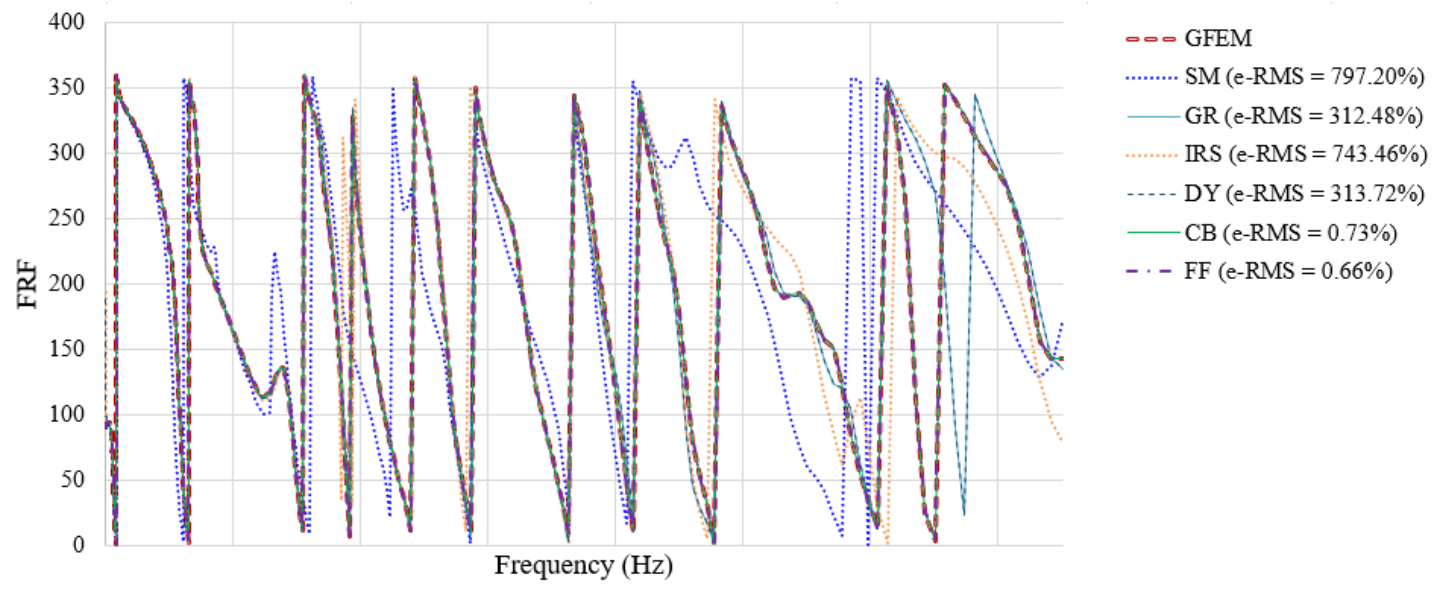

Figure 3.13 Comparison frequency response function (phase) for all six ROMs and GFEM

\subsection{Modal Participation}

The MPF [108] for all flexible modes between the frequency range of interest in the dynamic aeroelasticity response analysis (TDG case) is compared for all the ROMs and GFEM. MPF is found out at grid point ID 1019 along T2 direction at time $0.16 \mathrm{~s}$ as shown in Figure 3.14. A similar trend can be observed with highest RMS error is seen in SM, GR, IRS and DY. On the other hand, the ROMs based on CMS method is in good agreement with GFEM in terms of the MPFs extracted.

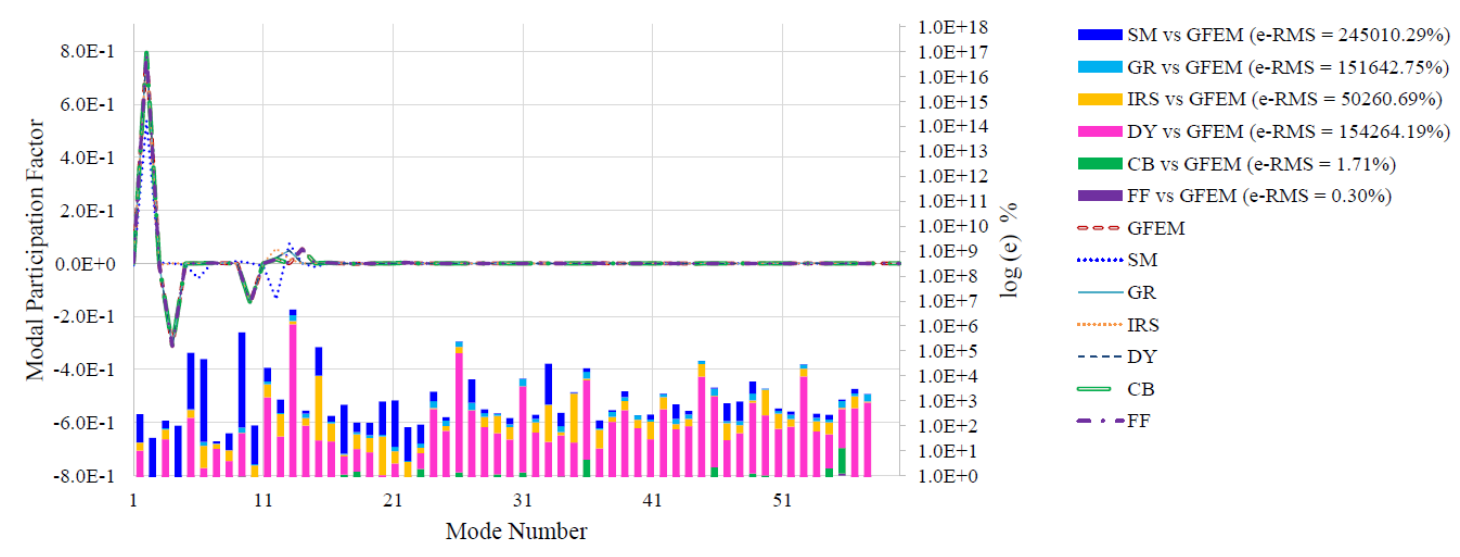

Figure 3.14 Comparison of Modal Participation Factor for all six ROMs with GFEM 
There are total 60 modes in the frequency range of interest and among this, only 5 dominant modes which participate in the dynamic aeroelasticity analysis of the GFEM. The percentage of error shown for each ROM includes the error from the nonsignificant and dominant modes. Since there are total 55 nonsignificant elastic modes in the error calculation, the reduced models such as SM, GR, IRS and DY ROMs show a higher percentage of error due to its poor representation of these nonsignificant modes in comparison to GFEM.

\subsection{ROM Convergence Speed}

The convergence speed of the deformation response of the different ROMs as compared to those of the GFEM is investigated with respect to the number of retained grid points employed in the ROM formation. Here, a standalone aircraft wing with 20 stations is considered for this analysis. The number of retained grid points are varied from 3 to 20 points through 8 different iterations as indicated in Table 3.4. A frequency response analysis using these models was performed and the deformation response corresponding to grid point with ID 1019, as shown in Figure 3.10, is recovered and compared. The RMS of the percentage of error between the frequency response of each ROM iteration and that of the GFEM is calculated and plotted in Figure 3.15. It can be seen that the frequency response for CMS ROMs match closely those of the GFEM at all iterations investigated. On the other hand, GR and DY ROMs are found to have almost the same convergence rate. It can also be seen that the SM and IRS ROM converge to a final solution that is far off from the GFEM result. This analysis indicates that CMS methods are independent on the number of grid points retained in the system, this is due the fact that there are 100 dominant 
modes corresponding to the interior DoFs included in both $\mathrm{CB}$ and FF ROM for all iterations.

Table 3.4 Convergence Speed Iteration

\begin{tabular}{cc}
\hline Iterations & Retained Grid Points \\
\hline 1 & 3 \\
2 & 5 \\
3 & 8 \\
4 & 10 \\
5 & 15 \\
6 & 17 \\
7 & 18 \\
8 & 20 \\
\hline
\end{tabular}

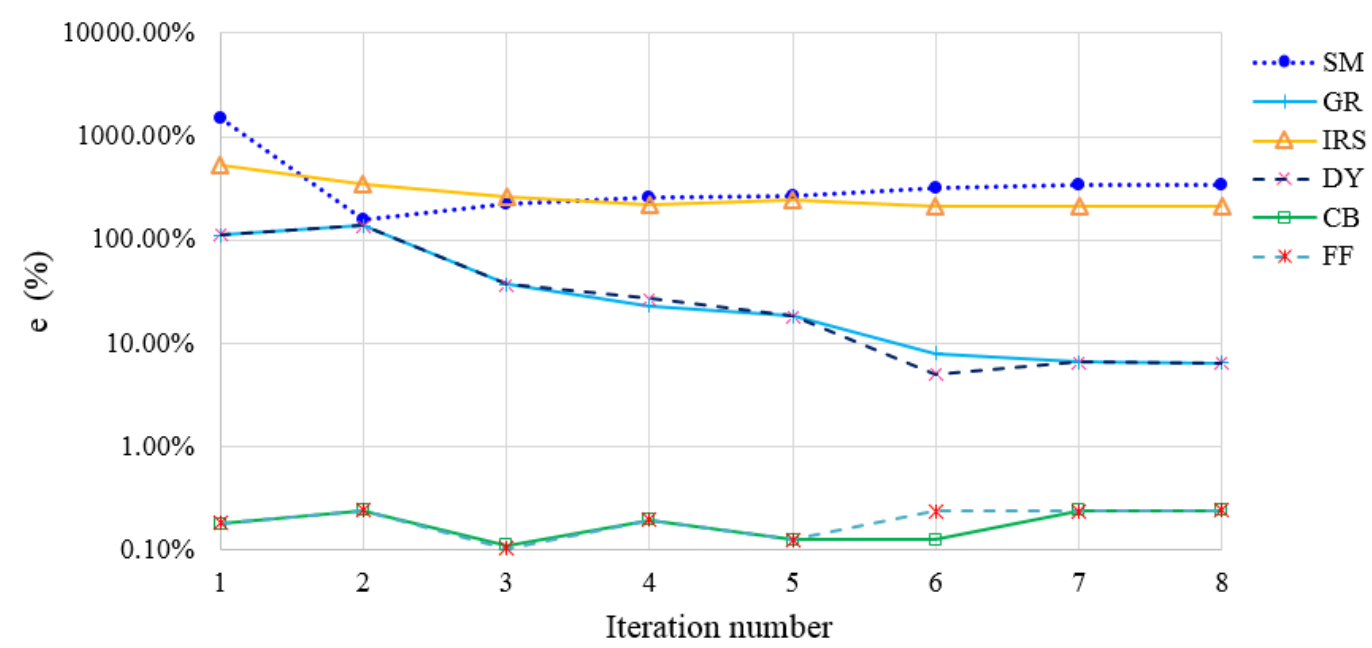

Figure 3.15 Percentage of error in frequency response of ROMs with respect to number of their retained grid points as compared to GFEM counterpart

The RMS errors associated with all the ROMs based on different analysis criteria investigated in the current case study are summarized as shown in Table 3.5. It can be found that the accurate MOR methodology which can be used for the aeroelasticity load analysis is CB and FF ROMs which have the least error possible. 
Table 3.5 Summary of RMS error associated with each analysis

\begin{tabular}{ccccccc}
\hline & \multicolumn{5}{c}{ e-RMS (\%) } \\
\cline { 2 - 6 } ROM & $\begin{array}{c}\text { Normal } \\
\text { mode } \\
\text { analysis }\end{array}$ & $\begin{array}{c}\text { Static } \\
\text { Aeroelasticity }\end{array}$ & TDG & $\begin{array}{c}\text { PSD } \\
\text { (Mag) }\end{array}$ & $\begin{array}{c}\text { PSD } \\
\text { (Phase) }\end{array}$ & MPF \\
\hline SM & 12.1 & 33.83 & 345.35 & 54.52 & 797.2 & 245010.29 \\
GR & 0.921 & 0.43 & 24.92 & 10.23 & 312.48 & 151642.75 \\
IRS & 2.66 & 0.86 & 120.78 & 17.38 & 743.46 & 50260.69 \\
DY & 0.92 & 0.51 & 22.81 & 14.87 & 313.72 & 154264.19 \\
CB & 0.000426 & 0.43 & 1.17 & 0.56 & 0.73 & 1.71 \\
FF & 0.0000616 & 0.43 & 1.14 & 0.75 & 0.66 & 0.3 \\
\hline
\end{tabular}

Table 3.6 ROMs generation cost

\begin{tabular}{cc}
\hline ROM & Generation cost (s) \\
\hline SM & 3007.080 \\
GR & 1285.282 \\
IRS & 3036.883 \\
DY & 1340.740 \\
CB & 4454.242 \\
FF & 4436.962 \\
\hline
\end{tabular}

Table 3.7 GFEM and ROMs analysis cost per aeroelasticity iteration

\begin{tabular}{cccc}
\hline & \multicolumn{3}{c}{ Analysis cost (s) } \\
\cline { 2 - 4 } Model & $\begin{array}{c}\text { Static } \\
\text { Aeroelasticity }\end{array}$ & \multicolumn{2}{c}{$\begin{array}{c}\text { Dynamic } \\
\text { Aeroelasticity }\end{array}$} \\
\cline { 2 - 4 } & & TDG & PSD \\
\hline GFEM & 42.435 & 441.722 & 75.071 \\
SM & 2.917 & 21.680 & 13.035 \\
GR & 13.054 & 25.402 & 23.443 \\
IRS & 13.068 & 26.443 & 23.155 \\
DY & 13.176 & 26.137 & 23.304 \\
CB & 14.731 & 27.564 & 25.690 \\
FF & 14.786 & 27.260 & 25.271 \\
\hline
\end{tabular}


ROM generation cost associated with each MOR methodologies considered under case study is indicated in Table 3.6. Here the generation cost for reduced matrix ROM includes reading the full GFEM mass and stiffness matrices, linear algebraic reduction operations as per MOR methodology and then writing down in a suitable reduced matrix format to import into FEM software like MSC NASTRAN. ROM based on GR have the least generation cost followed by DY, SM, IRS, FF, and CB. These generated ROMs are subjected to thousands of load cases to compute the critical loads envelope. Static and dynamic aeroelasticity load analysis time required for a single load case in MSC NASTRAN for the GFEM and ROMs is shown in Table 3.7. It can be seen that the all ROMs are fast and efficient compared to the GFEM with SM ROM being the cheapest out of all ROMs. On the other hand, the analysis time of the CMS ROMs are 16 and 2.9 times cheaper than the reference GFEM in the aeroelasticity analysis with TDG and PSD cases respectively. It should be noted that the reduction and aeroelasticity analyses were performed in a workstation with quad core $3.40 \mathrm{GHz}$ processor and 16 GB RAM. 


\section{Hybrid Stick Model}

As discussed in chapter 2 and chapter 3, SM development method generates low fidelity ROM with several inaccuracies which results in unrealistic stiffness distribution along the approximated elastic axis. However, SM offers an intuitive spatial representation of aircraft mass and stiffness distributions along the airframe elastic axis which is an important feature for aircraft loads analysis engineers.

On the other hand, Matrix-based ROMs possess high fidelity static and dynamic characteristics as compared to their GFEM counterparts within a frequency range of interest. However, several of the matrix-based MOR methods are represented in the mathematical modal domain which are deemed incongruous for aeroelasticity analyses as aircraft flight conditions are normally described in the physical space. While the GR and CB matrix-based ROMs are expressed in the physical space, these methodologies don't offer the handling flexibilities of the SM and as such, they are not favored by the aerospace industry.

In this section, a modified MOR methodology that is based on a hybrid SM (HSM) representation is presented. Here, the $\mathrm{SM}$ is augmented by residual mass and stiffness matrices that account for the aforementioned deficiencies of the base SM. As shown in Figure 4.1, steps of the HSM development process include i) defining a set of grid points along the airframe elastic axis that will serve as the retained DoFs of the GFEM, ii) developing a SM employing the set of retained DoFs defined in (i) and then computing the global mass and stiffness matrices of the developed SM iii) developing a matrix-based ROM employing the retained DoFs defined in (i), iv) generating the residual mass and 
stiffness matrices by computing the algebraic difference of the corresponding matrices defined in (ii) and (iii), and v) augmenting the developed mass and stiffness matrices computed in (iv) to the base SM defined in (ii) to form the HSM.

In the following subsections, we demonstrate the methodology employing a SM and its GR and CB ROMs counterparts.

\subsection{Residual Matrices of the HSM based on GR ROM}

The GR ROM stiffness and mass matrices are represented in the same physical coordinate as that of the SM. As both models are developed for the same DoFs of the retained grid point of the GFEM, hence the GR based residual mass and stiffness matrices are computed as,

$$
\mathrm{K}_{G R}^{R}=\mathrm{K}_{G R}-\mathrm{K}_{S M}
$$

$$
\mathbf{M}_{G R}^{R}=\mathbf{M}_{G R}-\mathbf{M}_{S M}
$$

where $\mathbf{K}_{G R}^{R} \in \mathbb{R}^{n_{r} \times n_{r}}$ and $\mathbf{M}_{G R}^{R} \in \mathbb{R}^{n_{r} \times n_{r}}$ denote the residual stiffness and mass matrices after the subtraction of SM stiffness from the corresponding GR ROM matrices.

These residual stiffness and mass matrices will be augmented to the base SM to form HSM ROM based on GR. In the rest of this thesis, we will abbreviate this model as HGR. 


\subsection{Residual Matrices for HSM based on CB ROM}

The stiffness and mass matrices of the CB ROM are expressed in a combination of physical and modal coordinates as shown in Eq. (2.45) and Eq. (2.46). The CB ROM submatrices expressed in the physical coordinate include $\mathbf{K}_{\boldsymbol{G R}}$ and $\mathbf{M}_{\boldsymbol{G R}}$. The algebraic difference between the CB ROM sub-matrices in the physical coordinate and the base SM matrices are equal to the $\mathbf{K}_{G R}^{R}$ and $\mathbf{M}_{G R}^{R}$ given in Eq. (4.1) and Eq. (4.2), respectively.

In the next step, the $\mathrm{CB}$ stiffness and mass sub-matrices corresponding to the modal coordinates are added to residual $\mathbf{K}_{G R}^{R}$ and $\mathbf{M}_{G R}^{R}$ matrices, respectively. Accordingly, the residual stiffness and mass matrices corresponding to the HSM based on CB ROM are given by,

$$
\begin{gathered}
\mathbf{M}_{C B}^{R}=\left[\begin{array}{cc}
\mathbf{I} & \left(\mathbf{M}_{i b}\right)_{d} \\
\left(\mathbf{M}_{b i}\right)_{d} & \mathbf{M}_{G R}^{R}
\end{array}\right] \\
\mathbf{K}_{C B}^{R}=\left[\begin{array}{cc}
(\boldsymbol{\Lambda})_{d} & \mathbf{0} \\
\mathbf{0} & \mathbf{K}_{G R}^{R}
\end{array}\right]
\end{gathered}
$$

where $\mathbf{M}_{C B}^{R} \in \mathbb{R}^{n_{r-C B} \times n_{r-C B}}$ and $\mathbf{K}_{C B}^{R} \in \mathbb{R}^{n_{r-C B} \times n_{r-C B}}$ are the CB residual mass and stiffness matrices, respectively, based on a combination of physical and modal coordinates.

Finally, the residual matrices in Eq. (4.3) and (4.4) are augmented to the SM ROM to form the HSM ROM based on CB. For convenience we will abbreviate this model as HCB ROM. 


\subsection{Dynamic Fidelity of HSMs: a Case Study}

This section presents a case study in which the conventional SM and HSM ROMs are employed in the modal and the dynamic aeroelasticity analysis of a Bombardier Aircraft platform. Figure 4.1 shows a schematic drawing of the model reduction process conducted in this section. Here, the aircraft GFEM is reduced to a SM represented by a series of beam elements extending along the airframe elastic axis. On the other hand, the same grid points used to define the stations of the SM ROM are employed as a cloud of grid points with the retained DoFs for the GR and CB ROMs. The mass and stiffness matrices of the base SM are then used with their corresponding counterparts of the GR and CB ROMs to compute the residual mass and stiffness matrices for the HSM. Finally, the computed residual matrices are augmented to base SM to form the HSMs.

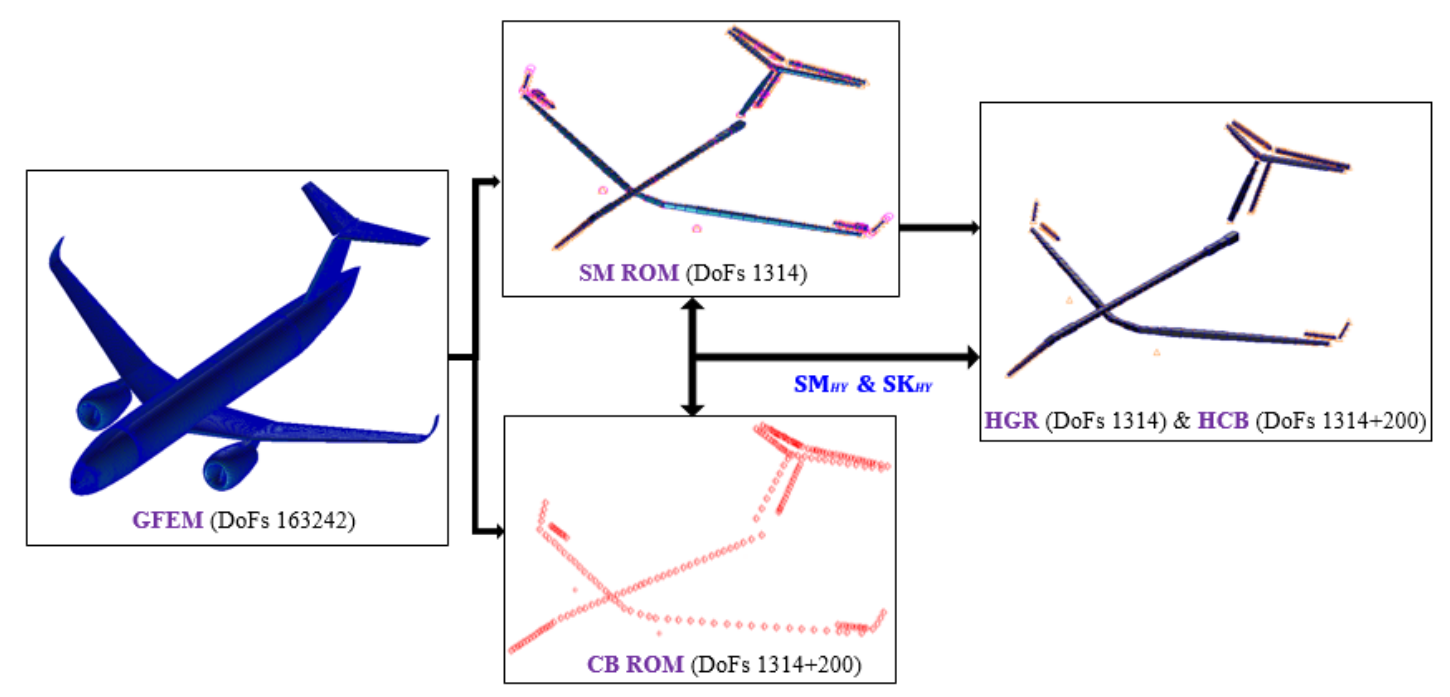

Figure 4.1 Visual representation of a generic aircraft HSM ROM 


\subsubsection{Normal Modes analysis}

A normal modes analysis is performed in MSC NASTRAN to compare the reduced models eigenvalues with those of the GFEM. A comparison of the percentage error, as per Eq. (3.3), of the eigenvalues corresponding to the first 10 flexible modes of the ROMs are shown in Figure 4.1. It can be observed from Figure 4.1 that the least error is associated with HCB and HGR ROMs. The maximum error is found in the base SM ROM with error root mean square value of $12.11 \%$ compared to the GFEM.

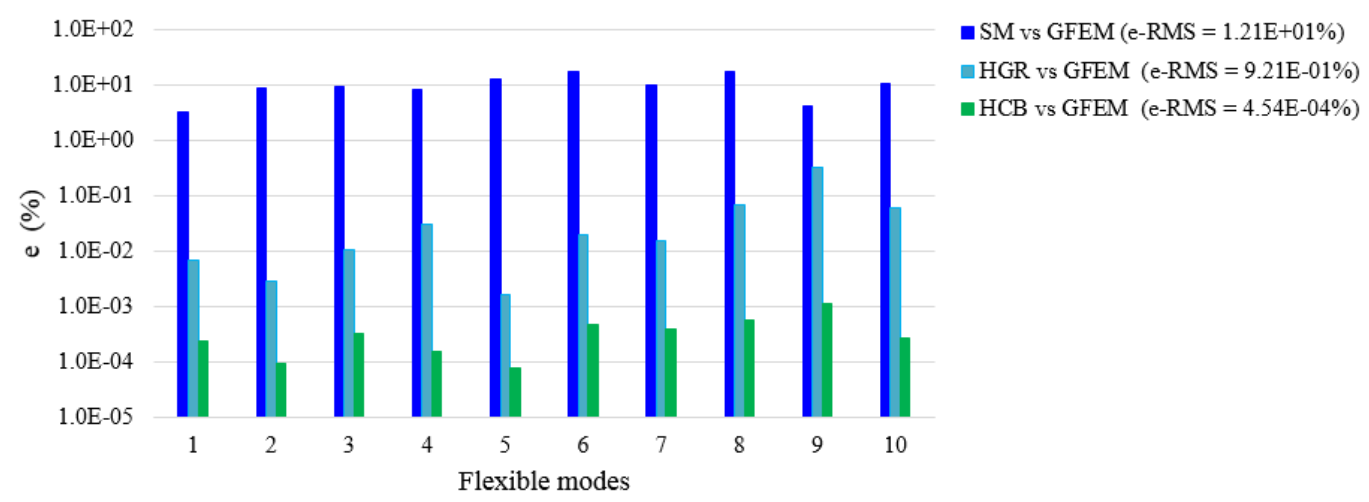

Figure 4.2 Percentage of error involved in the SM and HSM ROMs as compared to GFEM for the first 10 flexible eigenvalues

Modal Assurance Criterion (MAC) is also used to compare the eigenmodes of SM, HGR and HCB ROMs with the reference GFEM modes. As per this criterion, if the two mode shapes compared is identical then the MAC value will be close to 1 and if they are very different then MAC value will be close to zero [101]. In other words, MAC is the scalar constant that shows the degree of closeness among two eigenmodes being compared and it's calculated as, 


$$
\mathrm{MAC}=\frac{\left|\boldsymbol{\Phi}_{i-r}{ }^{T} * \boldsymbol{\Phi}_{G F E M-r}\right|^{2}}{\left\{\boldsymbol{\Phi}_{i-r}{ }^{T} \boldsymbol{\Phi}_{i-r}\right\}\left\{\boldsymbol{\Phi}_{G F E M-r}{ }^{T} \boldsymbol{\Phi}_{G F E M-r}\right\}}
$$

where $\boldsymbol{\Phi}_{i-r}$ is the $\mathrm{r}^{\text {th }}$ mode of the ROM and $\boldsymbol{\Phi}_{G F E M-r}$ is the $\mathrm{r}^{\text {th }}$ mode of the GFEM.

The first 14 normal modes are compared based on MAC value as shown in Figure 4.3 and Figure 4.4, below.

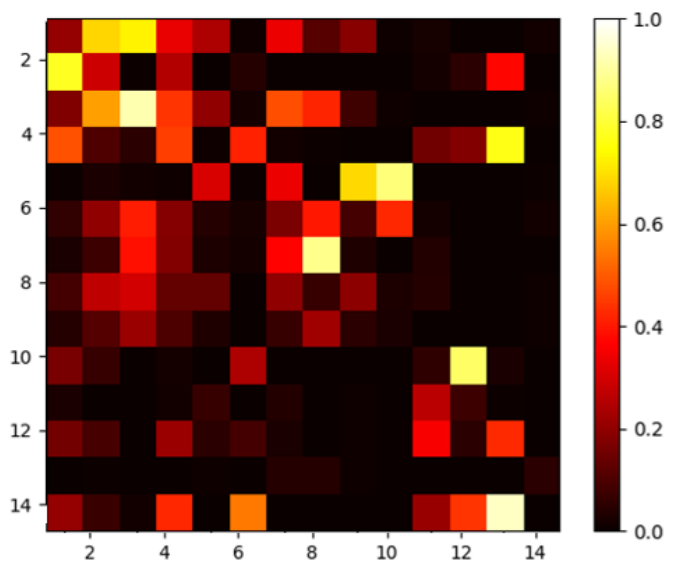

Figure 4.3 Normal modes comparison between SM ROM and GFEM based on MAC
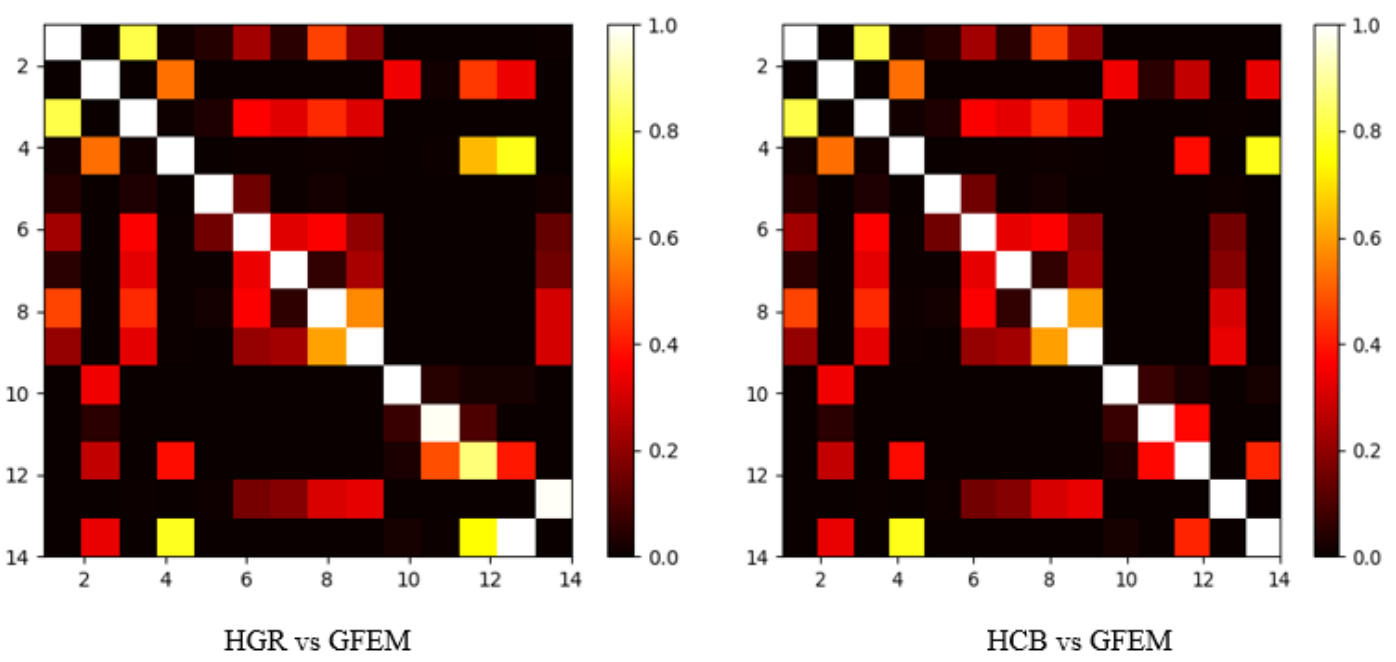

Figure 4.4 Normal modes comparison between HSMs and GFEM based on MAC

It can be seen that the eigenmodes of the SM ROM is very different as compared to the GFEM modes. Whereas the eigenmodes corresponding to the HGR and HCB ROM are 
in accordance with the GFEM modes. Though, the HGR modes starts deviating from GFEM modes starting at mode 11 because the static condensation is accurate near to zero frequency due to the ignored omitted DoFs inertial terms. It should be noted that the HGR and $\mathrm{HCB}$ are capable of exactly representing the bending and torsion coupled modes as seen in the GFEM.

\subsubsection{Dynamic aeroelasticity load analysis}

A dynamic aeroelasticity analysis is performed to check the accuracy of the dynamic loads recovered from the ROMs as compared to the GFEM for two load cases, namely, Tuned Discrete Gust (TDG) and Von-Karman Power Spectral Density (PSD) [105]. The dynamic loads are recovered from the retained grid ID 1019, which is located close to the tip of the left wing, with respect to the coordinate system ID 1 as shown in Figure 3.10.

\subsubsection{Recovered dynamic load in TDG gust case}

Dynamic aeroelasticity analysis is performed to measure the response of all the ROMs to a TDG case with flight conditions shown in Table 3.1.

The out of plane bending moment recovered from grid ID 1019 is shown in Figure 4.5. It can be observed from Figure 4.5 that the dynamic loads extracted from HCB conform very well with those of extracted from the GFEM using monitor points method [9] with a small e-RMS error of $0.89 \%$. Whereas the HGR ROM have an e-RMS error of $25.90 \%$. On the other hand, the moment recovered from SM is significantly deviated from that of the GFEM with e-RMS of $371.95 \%$. 


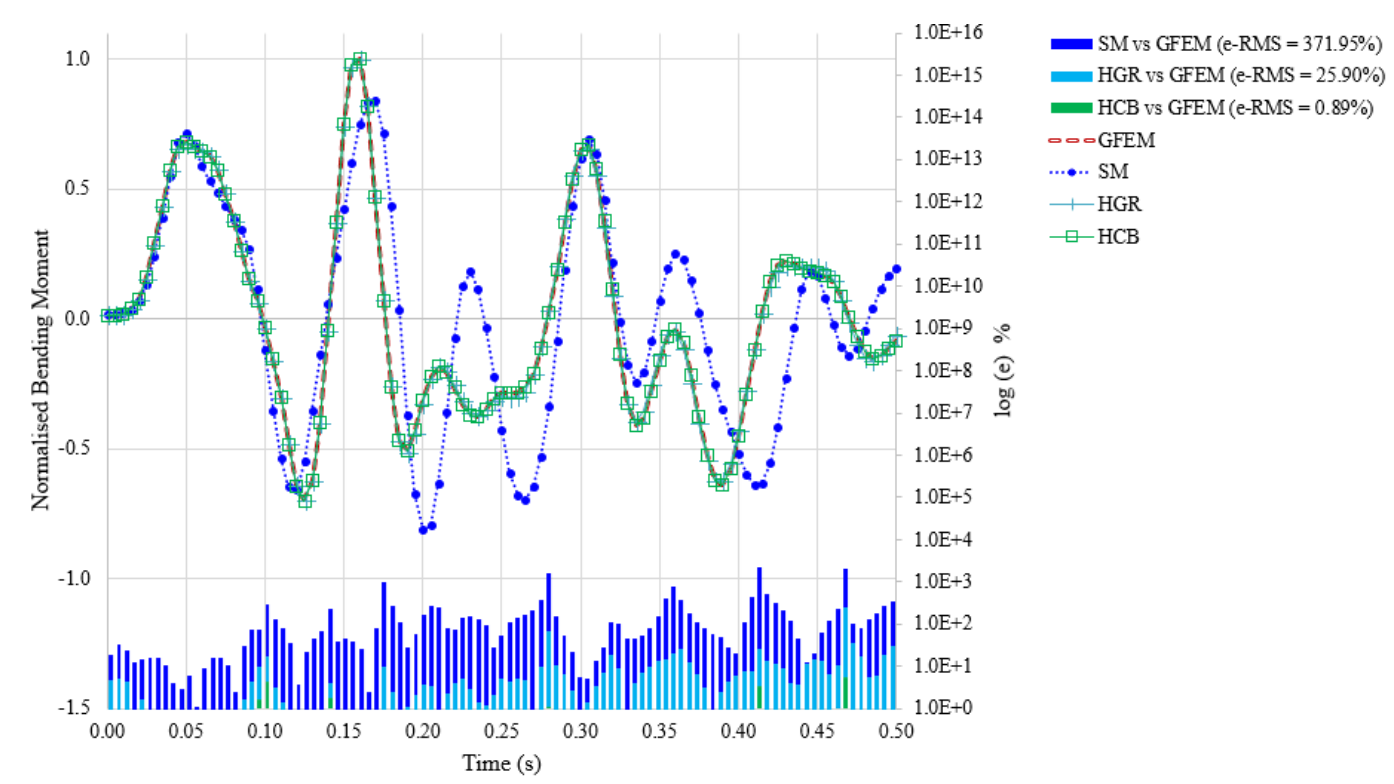

Figure 4.5 Comparison of out of plane bending moment recovered from SM, HSM ROMs and GFEM

\subsubsection{Recovered dynamic load in PSD vertical gust case}

A random response aeroelasticity analysis using Von Karman PSD case is performed. The gust properties and flight conditions used for the analysis is shown in Table 3.2 and Table 3.3, respectively. The frequency response function (FRF) magnitude and phase plots in the out of plane shear direction are shown in Figure 4.6 and Figure 4.7, respectively. The out of plane shear load recovered from the PSD gust analysis is represented in FRF of the structural response ([1], [106]).

It can be seen from Figure 4.6 and Figure 4.7 that the SM behavior is clearly not in agreement with the reference GFEM FRF with large e-RMS error of 57.13\% and $754.68 \%$ for magnitude and phase plots, respectively. Whereas, the dynamic loads extracted from the HCB ROM shows a good agreement with those of GFEM. Also, HGR ROM shows a very improved behavior as compared to the SM ROM with e-RMS error of only $10.31 \%$. 


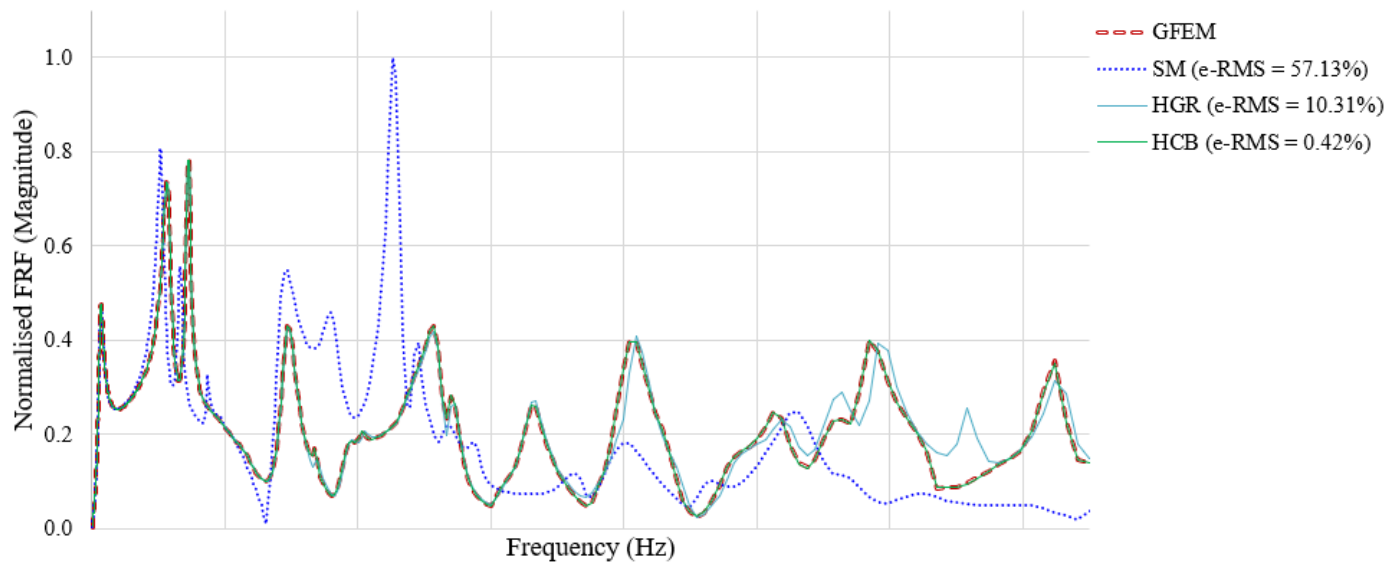

Figure 4.6 Comparison frequency response function (Magnitude) for SM, HSM ROMs and GFEM

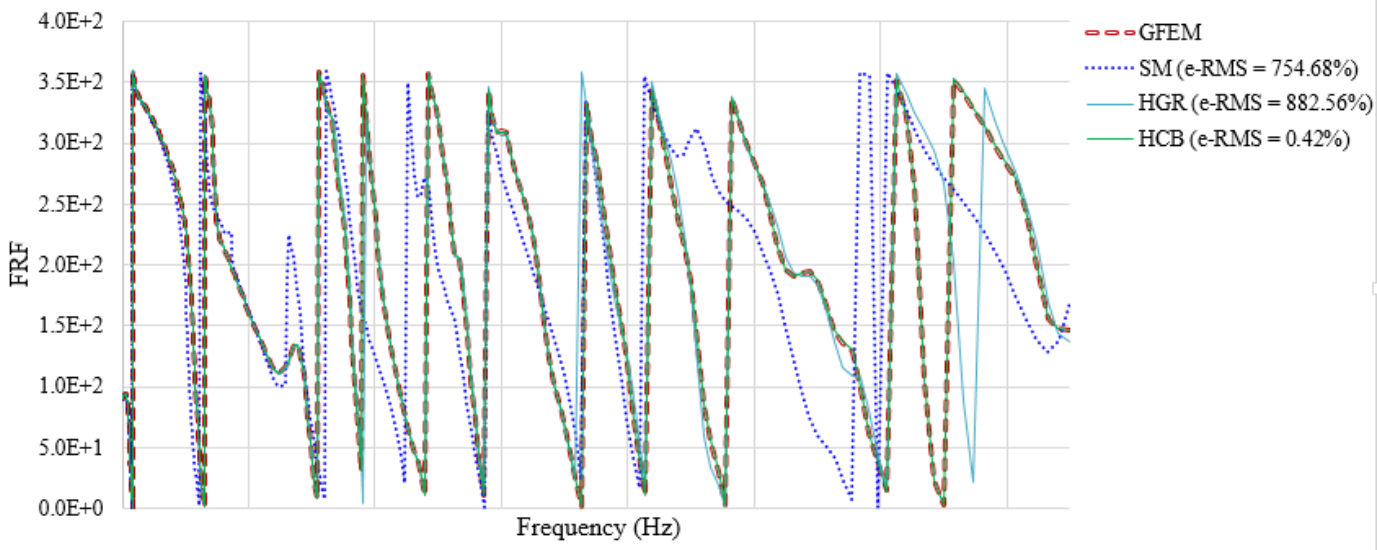

Figure 4.7 Comparison frequency response function (phase) for SM, HSM ROMs and GFEM

\subsubsection{Modal Participation}

The MPF for all flexible modes between the frequency range of interest in the dynamic aeroelasticity response analysis (TDG case) is compared for the HSM ROMs and GFEM. MPF is found out at grid ID 1019 along the translational DoF, T3, at time 0.165 second as shown in Figure 4.8. The major normal modes which participate in the response analysis of the GFEM include the elastic mode 2, 4 and 10. It can be seen that the HCB ROM modes 
participation shows the same behavior as the GFEM with a small error of $3.62 \%$. On the other hand, SM ROM has a higher RMS error which shows its poor agreement with the GFEM. The RMS error seen in the HGR ROM is also slightly on higher side even though the overall response for the TDG is almost matching with that of the GFEM.

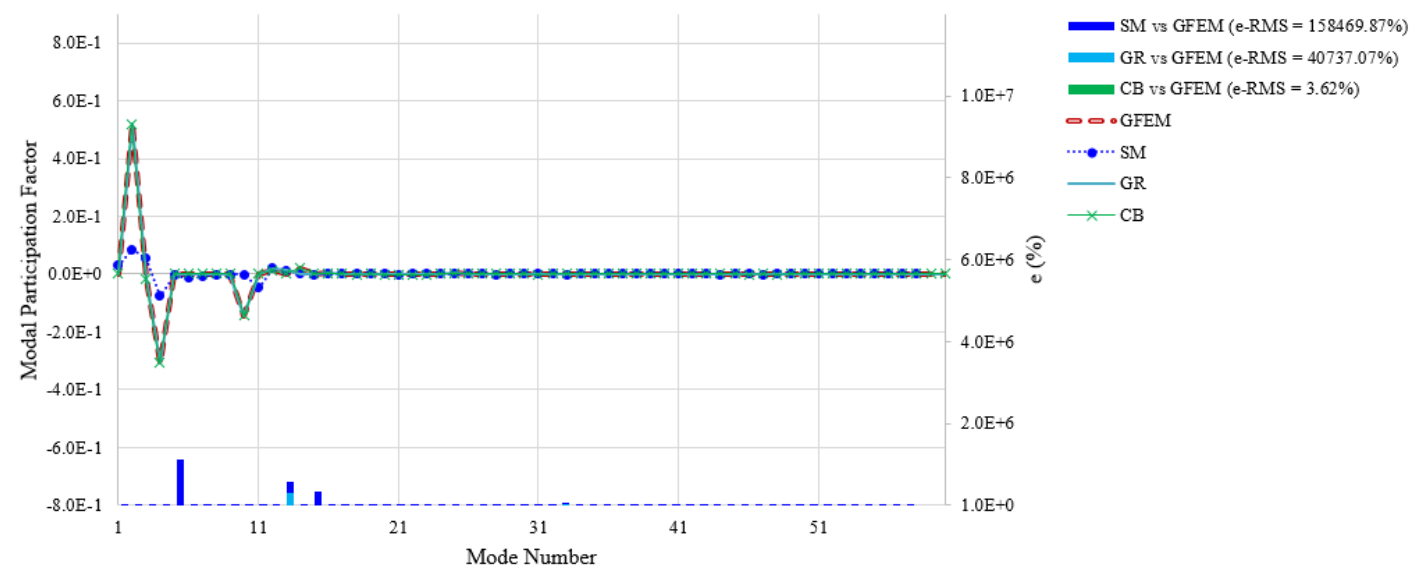

Figure 4.8 Comparison of Modal Participation Factor for SM and HSM ROMs with GFEM

The RMS error associated with all ROMs based on different analysis criteria investigated in the current case study is summarized as shown in Table 4.1. It can be seen that the HCB ROM followed by HGR ROM are having the least error in all analysis checks performed as compared to the GFEM. 
Table 4.1 Summary of RMS error associated with SM and HSM ROMs for each analysis

\begin{tabular}{ccccc}
\hline \multirow{2}{*}{ ROM } & \multicolumn{4}{c}{ e-RMS (\%) } \\
\cline { 2 - 5 } & Normal mode & \multicolumn{3}{c}{ Dynamic Aeroelasticity } \\
\cline { 2 - 5 } & analysis & TDG & PSD (Mag) & PSD (Phase) \\
\cline { 2 - 5 } SM & 12.095 & 371.95 & 57.13 & 754.68 \\
HGR & 0.921 & 25.90 & 10.31 & 882.56 \\
HCB & 0.000454 & 0.89 & 0.42 & 0.42 \\
\hline
\end{tabular}

Computational time requirement for the dynamic aeroelasticity load analysis for a single load case in MSC NASTRAN for the GFEM and ROMs is shown in Table 4.2. It can be seen that all ROMs are fast and efficient compared to the GFEM.

Table 4.2 GFEM, SM and HSM ROMs analysis cost per aeroelasticity iteration

\begin{tabular}{ccc}
\hline \multirow{2}{*}{ Model } & \multicolumn{2}{c}{ Analysis cost (s) } \\
\cline { 2 - 3 } & \multicolumn{2}{c}{$\begin{array}{c}\text { Dynamic } \\
\text { Aeroelasticity }\end{array}$} \\
\cline { 2 - 3 } & TDG & PSD \\
\hline GFEM & 441.722 & 75.071 \\
SM & 21.680 & 13.035 \\
HGR & 31.245 & 32.284 \\
HCB & 33.346 & 35.051 \\
\hline
\end{tabular}

\subsection{Handling Capabilities of HSMs}

In this section, we provide a hypothetical case study to demonstrate the handling capabilities of the HSM while maintaining its high dynamic fidelity compared to the SM. Here, we assume a design requirement to increase the stiffness properties of bay 8 of aircraft wing, as shown in Figure 4.9. Accordingly, a sensitivity analysis might be needed to study the effect of stiffness variation on the critical design load. Once the design stiffness parameters are determined, structures' engineers translate the stiffness change into new 
materials or geometrical modifications in the form of skin thickness, internal structural sizes and/or topology. To facilitate the comparison with the GFEM in this case study, we reverse here the process of bay 8 stiffness modification. Here, we initially vary the skin thickness of bay 8 of the GFEM and then we calculate the corresponding change in the stiffness properties of the corresponding SM. As shown in table 6, the shell elements forming the skin of wing bay 8 of the GFEM is initially assigned a constant thickness of 0.28 in. The residual mass and stiffness matrices of the HSM model are generated based on $\mathrm{CB}$ method. Then the skin thickness is varied in 4 iterations as shown in Table 4.3. In all of these iterations, the residual matrices of the HCB model are kept constant while the stiffness properties of bay 8 of the two wings of the SM are evaluated which resulted in the increase in the changes in the stiffness properties reported in Table 4.3.

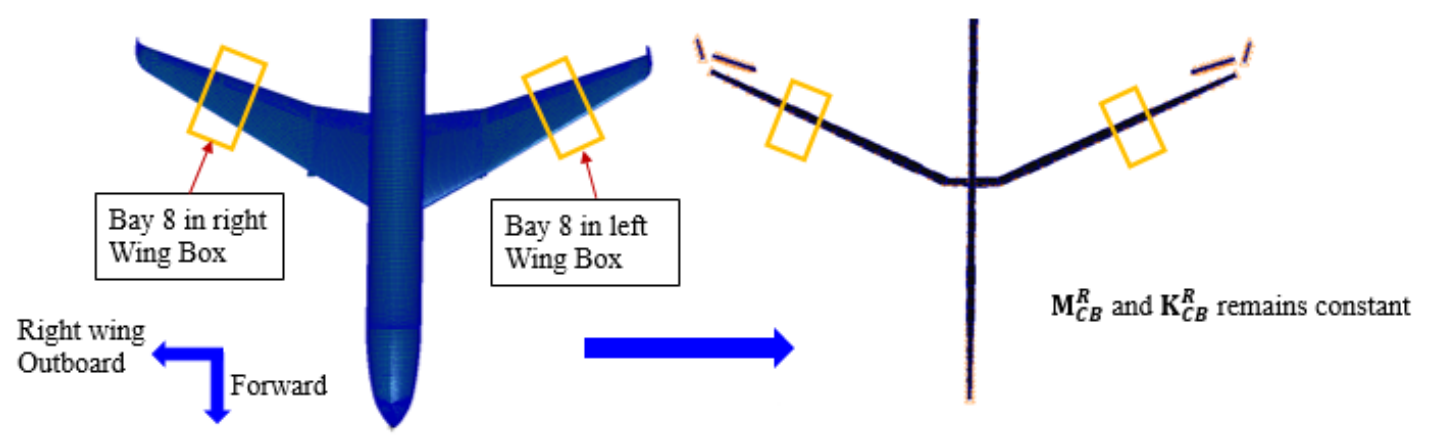

Figure 4.9 Skin thickness variation location for both GFEM and HCB ROM 
Table 4.3 Wing-box skin thickness variation

\begin{tabular}{cccccc}
\hline & $\begin{array}{c}\text { Increase in skin } \\
\text { Iterations } \begin{array}{c}\text { thickness of wing } \\
\text { bay 8 of the } \\
\text { GFEM (\%) }\end{array}\end{array}$ & \multicolumn{4}{c}{$\begin{array}{l}\text { Increase in SM beam stiffness } \\
\text { parameters of wing bay 8 (\%) }\end{array}$} \\
\cline { 3 - 6 } & $7 \%$ & 2.2 & 1.5 & 0.9 & 1.8 \\
2 & $15 \%$ & 4.6 & 3.2 & 1.9 & 3.7 \\
3 & $20 \%$ & 6.2 & 4.8 & 2.6 & 4.8 \\
4 & $30 \%$ & 9.3 & 6.5 & 3.8 & 6.9 \\
\hline
\end{tabular}

The GFEM and the HCB ROM are compared based on the normal modes and dynamic aeroelasticity loads. The eigenvalues and eigenvectors comparison of the 4 iterations are shown Figure 4.10 and Figure 4.11. It can be seen that HCB ROM in each iterations behave very well as compared to the GFEM in terms of the normal mode analysis results.

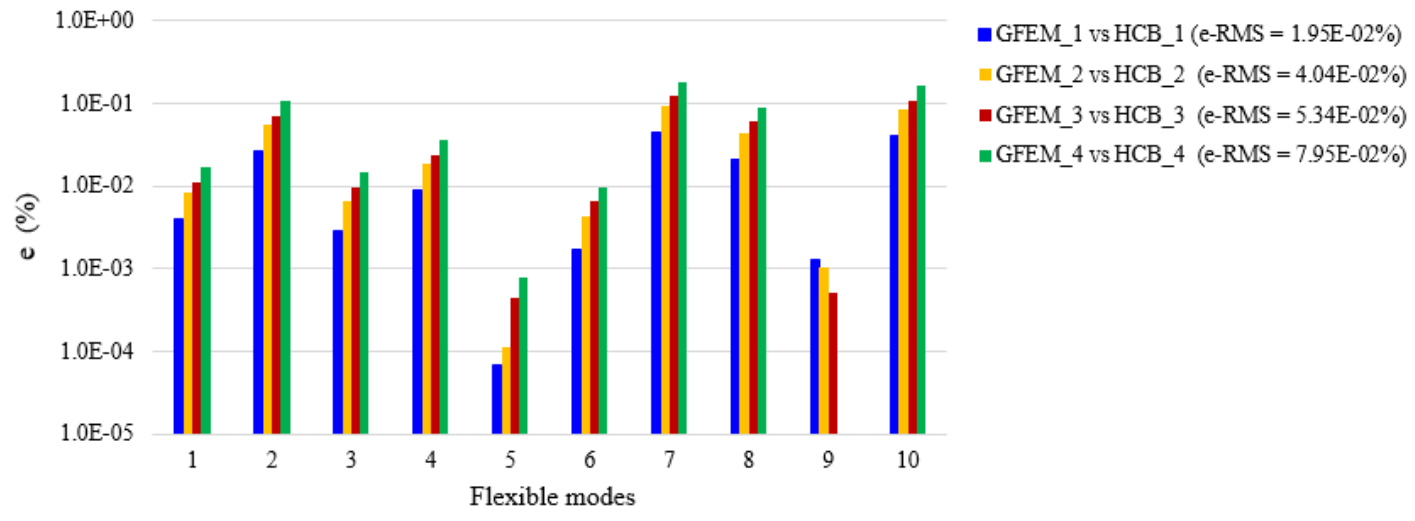

Figure 4.10 RMS error associated with the HCB ROM eigenvalues compared to GFEM in all iterations 


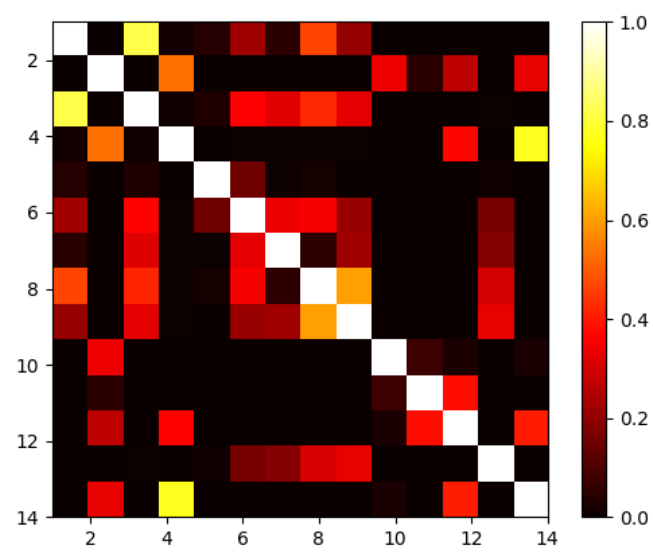

a) $1^{\text {st }}$ Iteration with $7 \%$ thickness

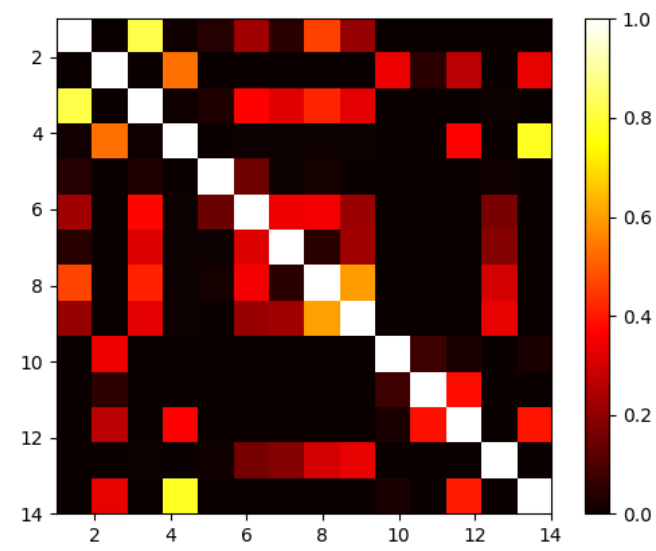

c) $3^{\text {rd }}$ Iteration with $20 \%$ thickness

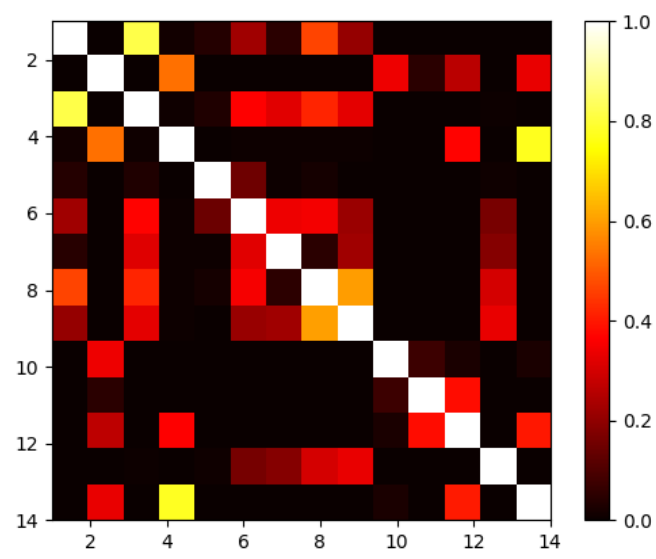

b) $2^{\text {nd }}$ Iteration with $15 \%$ thickness

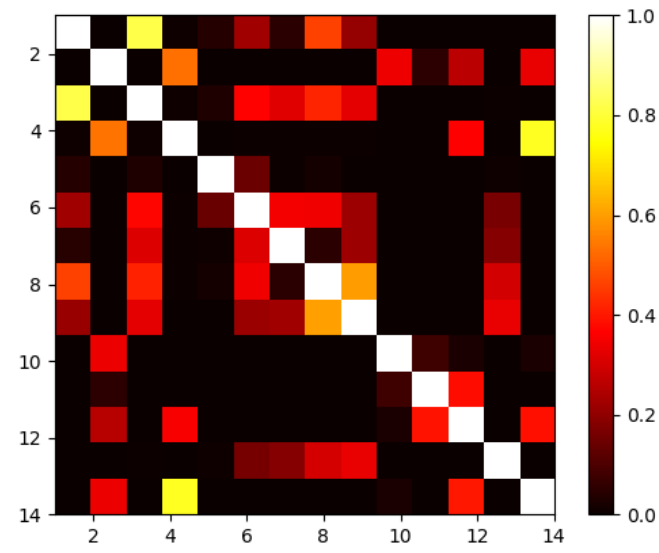

d) $4^{\text {th }}$ Iteration with $30 \%$ thickness

Figure 4.11 Normal modes comparison based on MAC for HCB and GFEM for all iterations

Dynamic aeroelastic loads at grid point 1019, shown in Figure 3.10, are calculated in TDG and PSD gust conditions. We demonstrate the loads results for iterations 1 and 4 as shown in Figure 4.12, Figure 4.13, Figure 4.14 and Figure 4.15. It can be seen that the HCB ROM and GFEM loads are in excellent agreement which is in line with the normal modes analysis results. On the other hand, the SM generated a totally unreliable results. 


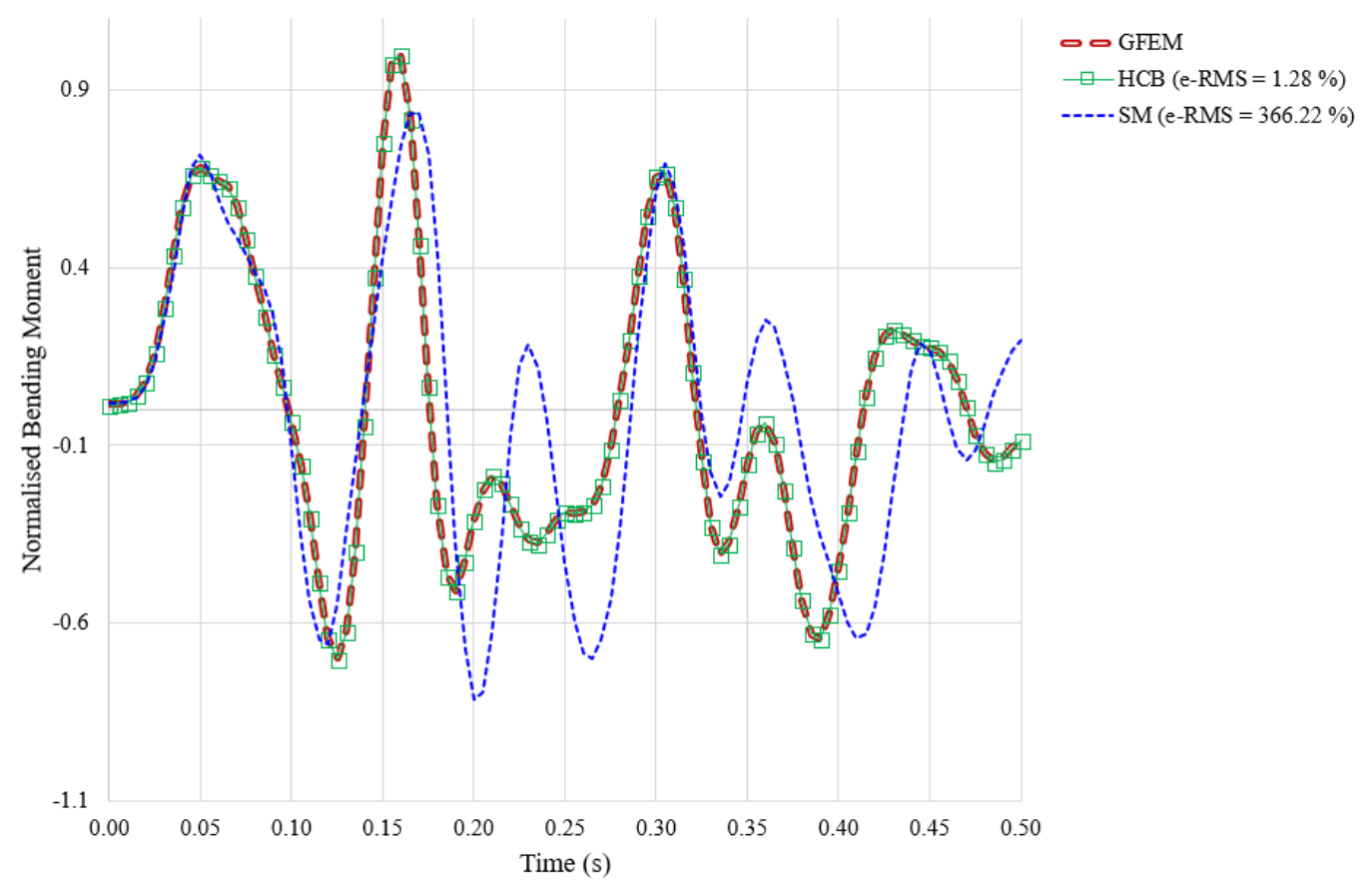

Figure 4.12 Comparison of out of plane bending moment (TDG case) for GFEM and $\mathrm{HCB}$ in iteration 1

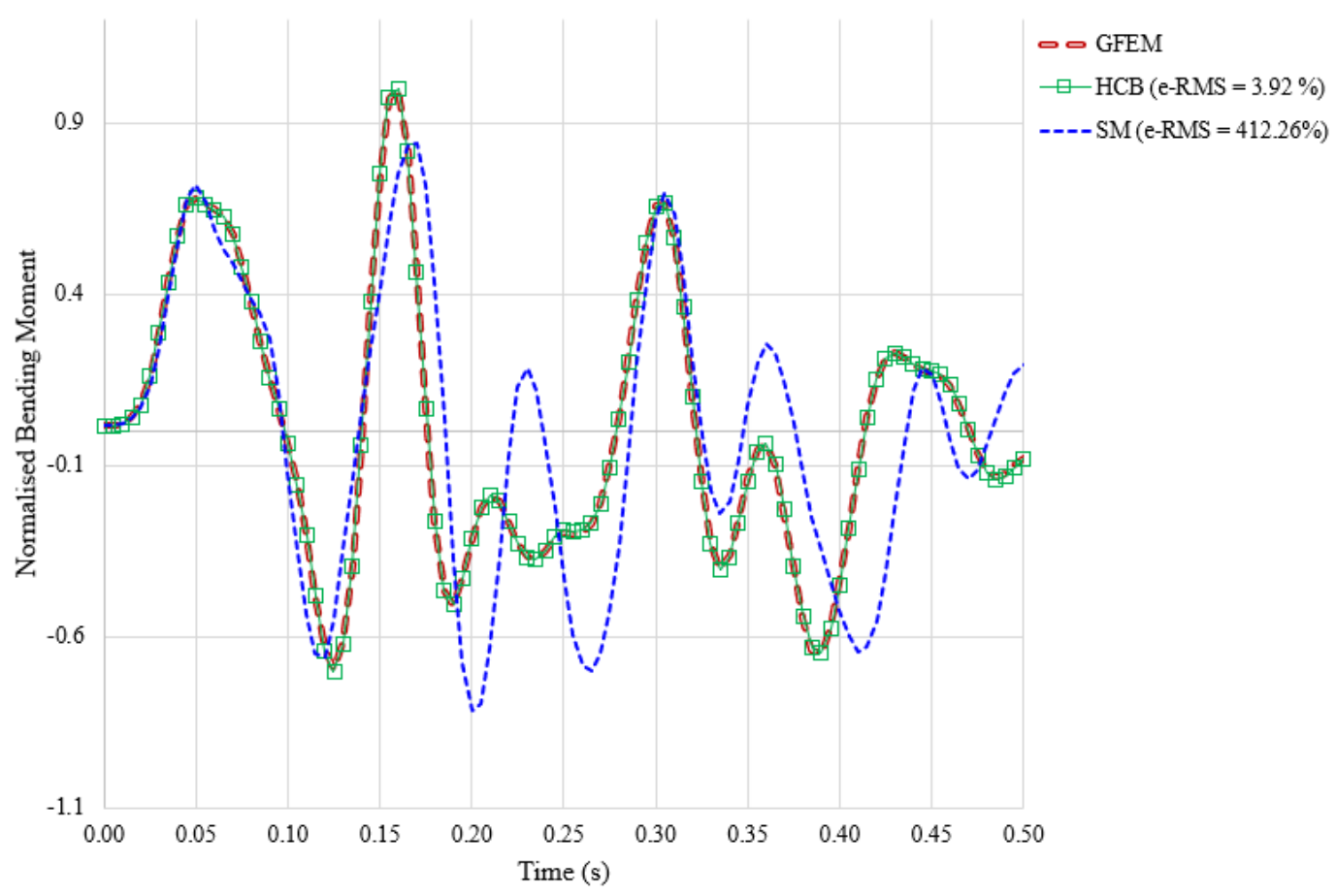

Figure 4.13 Comparison of out of plane bending moment (TDG case) for GFEM and HCB in iteration 4 


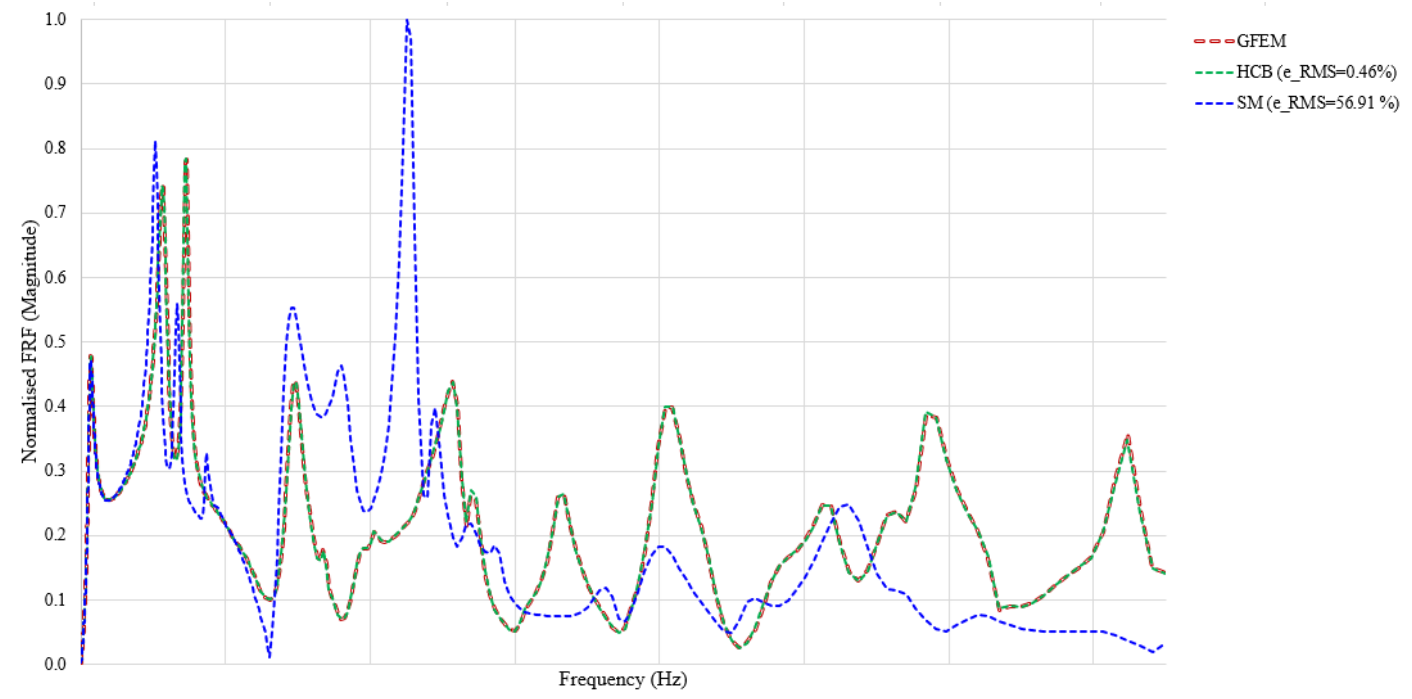

Figure 4.14 Comparison of the FRF (PSD case) for GFEM and HCB in iteration 1

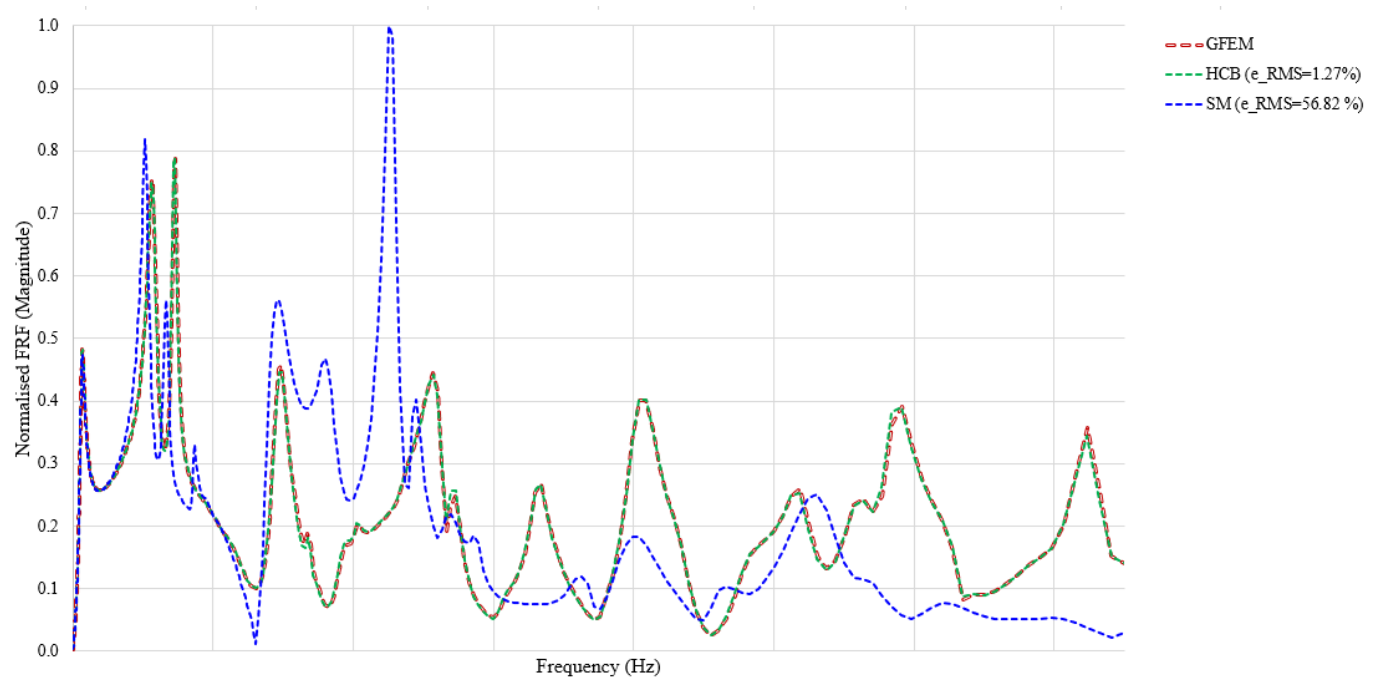

Figure 4.15 Comparison of the FRF (PSD case) for GFEM and HCB in iteration 4 


\section{Design optimization of aircraft Stick Model}

This section provides a detailed description of the SM optimization problem with the objective to match the modal performance of a set of selected modes in the GFEM within a frequency range of interest. For the optimization task, the SM ROM generated by unitary loading method is used as the base model. Firstly, the GFEM elastic axis is determined using the bisection approach [102]. Secondly, major modes that participate in the gust response analysis of the GFEM is identified. Thirdly, an optimization problem is defined to minimize errors in the selected modes of the SM as compared to their corresponding modes of the GFEM. The final product of the optimization problem is an OSM with dynamic characteristics closely matching the GFEM within the selected frequency range of interest.

\subsection{Elastic Axis Position Optimization}

An approximate elastic axis of a mechanical component is the line connecting the shear centers of different structural stations. The shear center is defined as the point at which an applied transverse loading results only in pure bending without twisting of the section. In this optimization problem, the elastic axis is computed by applying a unit shear force at every airframe station and recording the location where no twisting occurs using the bisection method [103]. Figure 5.1 shows the elastic axis determination of a 3 bay wingbox which consists of defining the search line from the leading edge $(\mathrm{C} 1)$ to the trailing edge (C2). A local coordinate system is defined with its origin at the mid-chord location between the $\mathrm{C} 1$ and $\mathrm{C} 2$ positions, with one axis along the search line, the second axis along the wing assumed torsional direction while the third axis is determined using the right hand 
rule. This local coordinate system is used as a reference to locate the loading point. Assuming that the positions of points $\mathrm{C} 1$ and $\mathrm{C} 2$ in the defined local coordinate system are $\xi \min =\mathrm{W} 1$ and $\xi \max =\mathrm{W} 2$, respectively. Accordingly, the search line initial interval will be $[\xi \min , \xi \max ]$. Then, the transverse force is applied at $\xi=(\xi \min +\xi \max ) / 2$. It should be noted that the loading point is connected to the surrounding grid points of the GFEM using Flexible MPC to transfer the applied loading to the surrounding structure of the wing-box [100]. Whereas, the retained grid point of the inboard end of the same wing bay is fixed in all DoFs and connected to the surrounding structure using Rigid MPC.

In the first iteration, the resulting torsional deformation at the loading grid point is measured. If the torsional deformation is negative, then the wing-box is twisted with leading edge upwards. Therefore, the point of force application is moved towards the trailing edge in the next iteration. Here, the search limits are updated where $\xi_{\min }=\xi$ while $\xi_{\max }$ remains the same as previous iteration. Accordingly, the force application location for the next iteration is then set to be $\left(\xi_{\min }+\xi_{\max }\right) / 2$.

On the other hand, if the torsional displacement is positive then, the wing-box will be twisted with trailing edge upwards. Therefore, the point of force application needs to be moved towards the leading edge. Accordingly, the search line interval is updated in the next iteration where $\xi_{\max }=\xi$ and $\xi_{\min }$ remains the same as previous iteration. Accordingly, the new force application location for the next iteration will follow same formulation as before, $\left(\xi_{\min }+\xi_{\max }\right) / 2$. 
The process is repeated until the minimum twist point is identified along the search line. This process is efficient and converges to the approximate elastic axis faster than exploring the full search line using brute force approach [103].

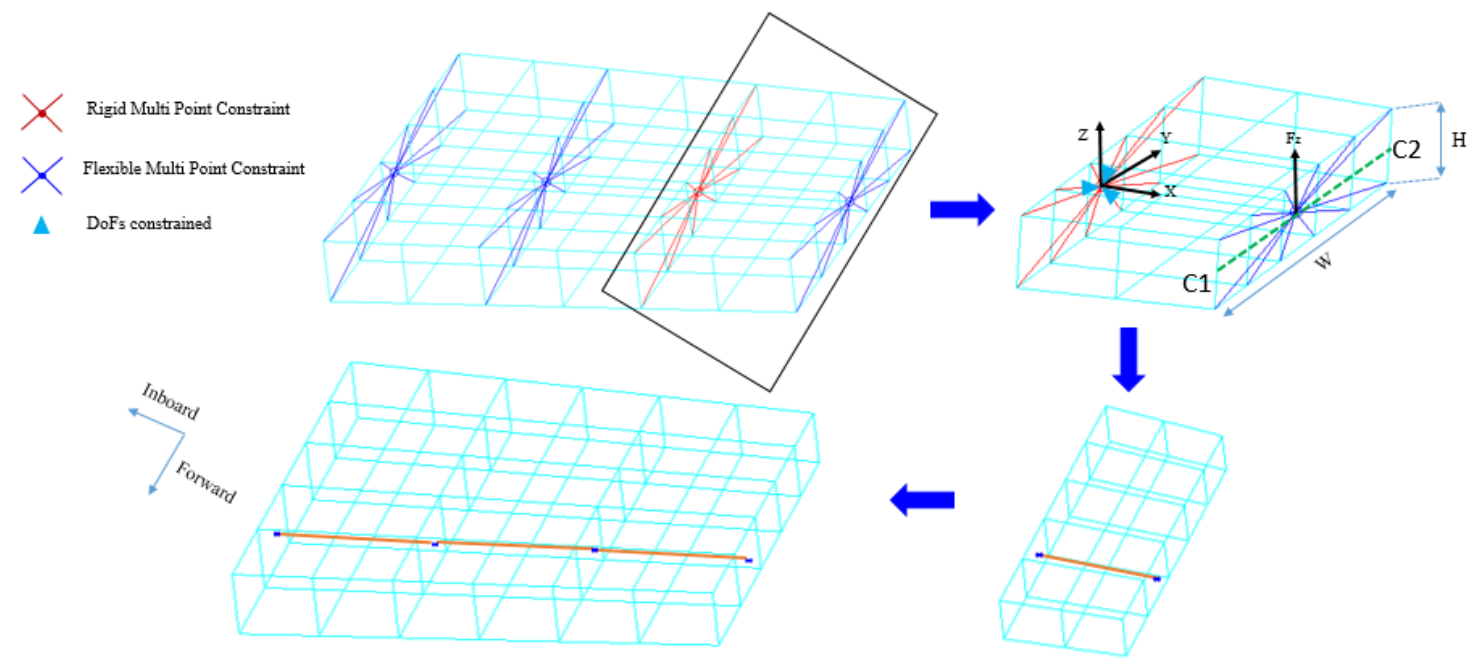

Figure 5.1 Wing-box elastic axis determination

\subsection{Stick Model Stiffness Parameters Optimization}

\subsubsection{Objective function}

The base SM is a series of Timoshenko beam element with no offset between the mass and the shear centers [109]. The beam parameters to be optimized include A, $I_{y}, I_{z}, J_{x}, K_{Y}$ and $K_{Z}$. Accordingly, the list of variables, $\mathbf{y}$, for the design optimization problem can be given as,

$$
\mathbf{y}=\left\{\mathrm{A}_{1},\left(I_{y}\right)_{1},\left(I_{z}\right)_{1},\left(J_{x}\right)_{1},\left(K_{Y}\right)_{1},\left(K_{Z}\right)_{1}, \ldots \ldots, A_{q},(I y)_{q},(I z)_{q},\left(J_{x}\right)_{q},\left(K_{Y}\right)_{q},(1\right.
$$

where subscript "q" denote the total number of beam elements in the SM ROM. 
The full stiffness matrix for a constant cross section Timoshenko beam element with two nodes of 6 DoFs each is shown in Appendix A. The upper and the lower bounds for the design space is restricted to $40 \%$ downward and $20 \%$ upward of the base SM ROM beam parameters. Those are the margins for the design variables identified by our industrial partner. The multi-objective functions for the optimization problem is formulated to minimize the errors in the modal pairs of the base SM as compared to the corresponding modes of the reference GFEM within a frequency range of interest. Comparison of normal modes is done by matching the respective eigenvalues and normalized mode shapes of the SM and the GFEM. The percentage error, $\left(\mathrm{e}_{N F}\right)_{r}$, in the natural frequency of $\mathrm{r}^{\text {th }}$ mode is given by,

$$
\left(\mathrm{e}_{N F}\right)_{r}=\left|\frac{\left(\omega_{\text {OSM }}\right)_{r}-\left(\omega_{\text {GFEM }}\right)_{r}}{\left(\omega_{\text {GFEM }}\right)_{r}}\right| \times 100
$$

where $\left(\omega_{O S M}\right)_{r}$ and $\left(\omega_{\text {GFEM }}\right)_{r}$ denote the $\mathrm{r}^{\text {th }}$ mode natural frequency of the OSM and the GFEM, respectively.

On the other hand, comparison of the eigenvectors requires the use of the MAC [101] as shown in Eq. (5.3),

$$
\mathrm{MAC}_{r}=\frac{\left|\boldsymbol{\Phi}_{\text {OSM }-r}{ }^{T} * \boldsymbol{\Phi}_{G F E M-r}\right|^{2}}{\left\{\boldsymbol{\Phi}_{\text {OSM }-r}{ }^{T} \boldsymbol{\Phi}_{\text {OSM }-r}\right\}\left\{\boldsymbol{\Phi}_{G F E M-r}{ }^{T} \boldsymbol{\Phi}_{G F E M-r}\right\}}
$$

where $\boldsymbol{\Phi}_{O S M-r}$ is the $\mathrm{r}^{\text {th }}$ mode of the OSM ROM and $\boldsymbol{\Phi}_{G F E M-r}$ is the $\mathrm{r}^{\text {th }}$ mode of the GFEM. $\boldsymbol{\Phi}_{O S M-r}{ }^{T}$ and $\boldsymbol{\Phi}_{G F E M-r}{ }^{T}$ are the $\mathrm{r}^{\text {th }}$ mode transpose of the OSM ROM and GFEM, respectively. 
The percentage of error in MAC, $\left(e_{M A C}\right)_{r}$, between the OSM and the GFEM in the $\mathrm{r}^{\text {th }}$ mode shape is calculated as,

$$
\left(e_{M A C}\right)_{r}=\left|\mathrm{MAC}_{r}-1\right| \times 100
$$

Thus the objective function can be formulated as,

$$
f(\mathbf{y})=\sum_{r=1}^{n_{p}}\left(\mathrm{e}_{N F}\right)_{r} *\left(e_{M A C}\right)_{r}+g\left(\left(\mathrm{e}_{N F}\right)_{r},\left(e_{M A C}\right)_{r}\right) * C(r)
$$

where $n_{p}$ is the number of selected GFEM modes for stick optimization. C is a penalty cost-value vector with values arranged in decreasing order. Also, $g\left(\left(\mathrm{e}_{N F}\right)_{r},\left(e_{M A C}\right)_{r}\right)$ is a Boolean function whose value will be either 0 or 1 depending on the $\left(\mathrm{e}_{N F}\right)_{r}$ and $\left(e_{M A C}\right)_{r}$. Accordingly, $g\left(\left(\mathrm{e}_{N F}\right)_{r},\left(e_{M A C}\right)_{r}\right)$ is defined as,

$$
g\left(\left(\mathrm{e}_{N F}\right)_{r},\left(e_{M A C}\right)_{r}\right)=\left(\begin{array}{cc}
0 & \text { if }\left(\mathrm{e}_{N F}\right)_{r}<4 \% \text { and }\left(e_{M A C}\right)_{r}<15 \% \\
1 & \text { if }\left(\mathrm{e}_{N F}\right)_{r} \geq 4 \% \text { and }\left(e_{M A C}\right)_{r} \geq 15 \%
\end{array}\right.
$$

In Eq. (5.5), the Boolean function and penalty cost-value vector gives more preference to the lower modes in the selected GFEM mode list.

Therefore, the bounded optimization problem can be defined as,

Minimize

subject to $f(\mathbf{y})$

$$
0.6 \mathbf{y}_{S M} \leq \mathbf{y} \leq 1.2 \mathbf{y}_{S M}
$$

where $\mathbf{y}_{S M}$ and $\mathbf{y}$ are the beam parameter vector corresponding to the base SM and the OSM ROM, respectively. While $\mathbf{y}$ is the vector of design variables representing the bounded beam parameters to be optimized, as given by Eq. (5.1). 
Here, the Differential Evolution (DE) algorithm [110] is used for the stick optimization to find the global minimum of the multivariate function. DE algorithm is stochastic in nature (does not use gradient methods) to find the global minima and can search a large design space.

\subsection{Dynamic Fidelity of the OSM: a Case Study}

This section presents a case study in which the conventional SM and the OSM ROM are employed in the modal and the dynamic aeroelasticity analysis of a Bombardier Aircraft platform.

\section{$\underline{\text { Modes Selection }}$}

A dynamic aeroelasticity analysis using TDG case is performed using GFEM to determine the dominant elastic modes which participate in the dynamic response. The dominant elastic modes identified based on the Modal Participation factors are used in the design optimization of the stick model. Here, for the MPF extraction purposes, a total 7 grid points considered which include 4 grid points along the aircraft wing ( 2 grid points from each left and right wing) and 3 grid points along the fuselage. Total 10 modes were selected from the GFEM which includes the 1st, 2nd and 3rd wing bending modes, 1st and 2nd fuselage torsional modes, 1st wing in-plane bending, 1st and 2nd wing bending and horizontal tail bending.

\subsubsection{Normal Modes analysis}

The comparison of percentage of error seen in the SM and the OSM ROM for the first 10 elastic modes eigenvalues is shown in Figure 5.2. It can be observed that the RMS error 
seen in OSM ROM (e-RMS $=4.36 \%$ ) is less than half the error seen in SM ROM (e-RMS $=12.11 \%)$. Mode shape comparison using MAC is shown in Figure 4.3 and Figure 5.3 for SM and OSM ROM, respectively. It can be seen that the first 10 elastic mode shapes of the OSM are in better agreement with the mode shapes of the GFEM compared to those of the base SM.

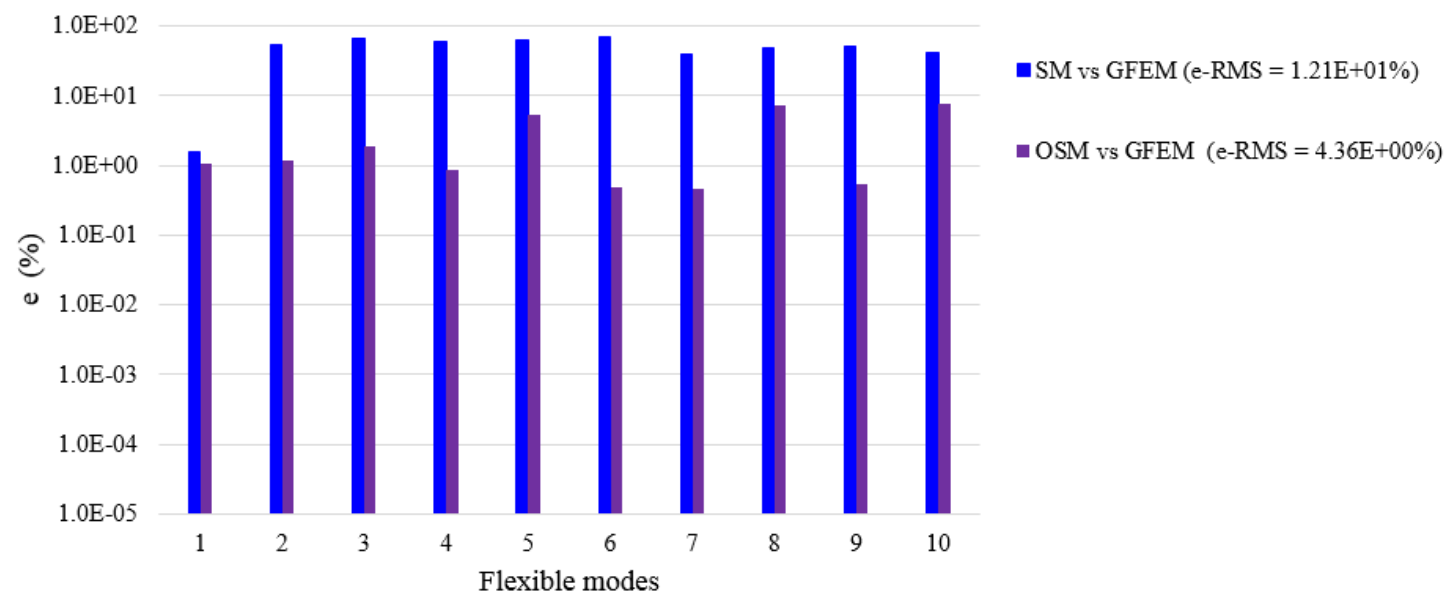

Figure 5.2 Percentage of error in SM and OSM ROMs as compared to GFEM for the first 10 flexible eigenvalues

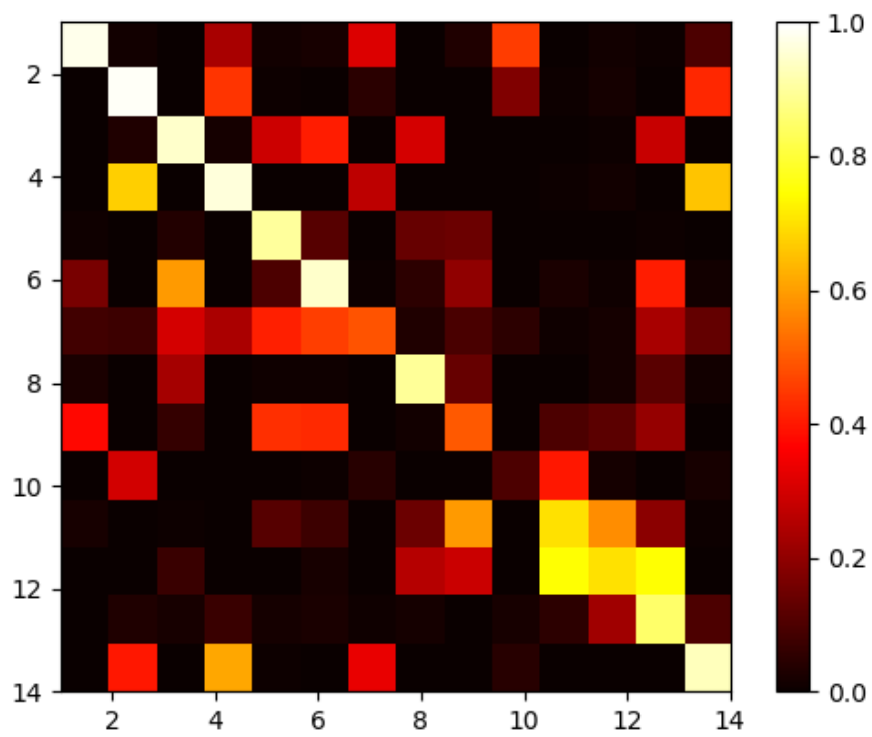

Figure 5.3 Normal modes comparison between OSM ROM and GFEM based on MAC 


\subsubsection{Dynamic aeroelasticity load analysis}

A dynamic aeroelasticity analysis, using the same flight and gust conditions presented in the case studies in chapter 3 and chapter 4 , is performed to check the accuracy of the dynamic loads recovered from the ROMs as compared to the GFEM for two gust cases, namely, TDG and Von-Karman PSD [105]. The dynamic loads are recovered from the retained grid ID 1015 with respect to the coordinate system ID 17 as shown in Figure 5.4.

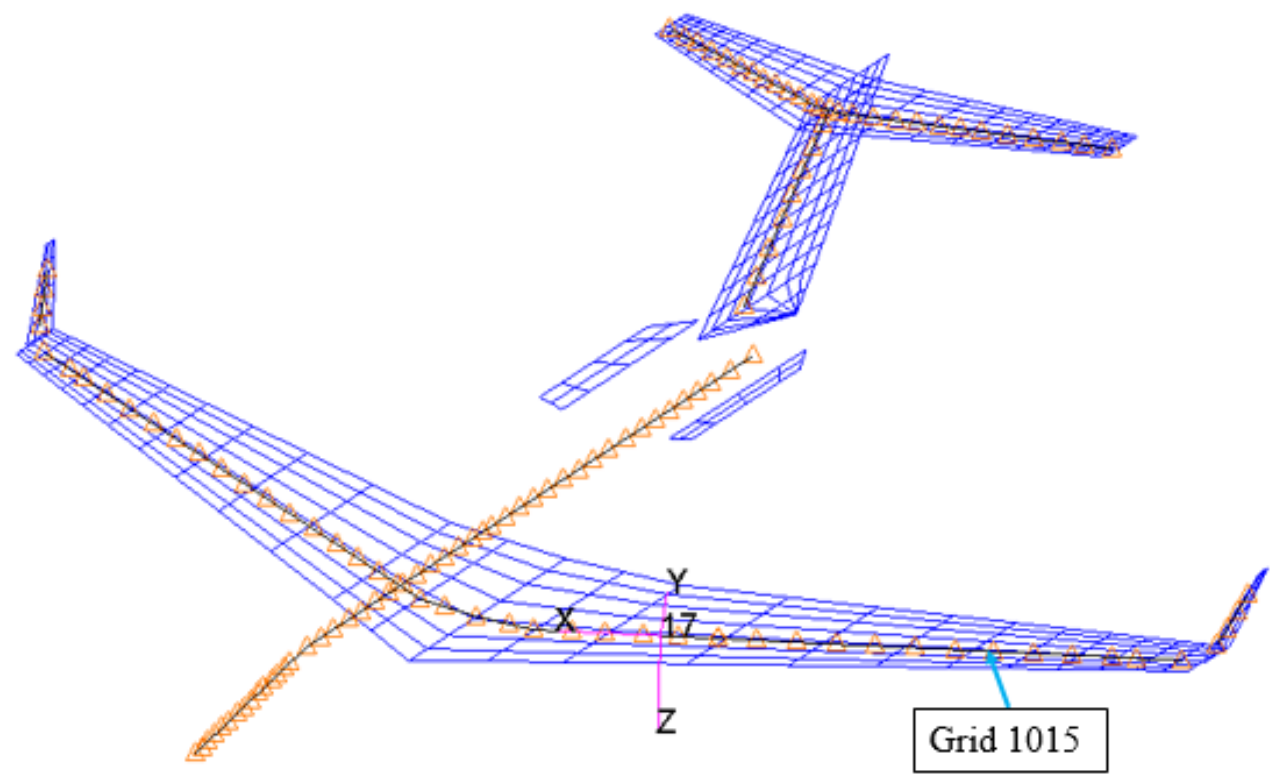

Figure 5.4 OSM and SM dynamic loads recovery grid point and coordinate

\subsubsection{Recovered dynamic load in TDG and PSD gust case}

It can be observed from the Figure 5.5 that the OSM ROM is in good agreement with e-RMS of $134.02 \%$ which is significantly less as compared to the SM ROM with e-RMS of $397.01 \%$. On the other hand, the loads recovered from OSM in PSD case show an improved behavior with only $21.14 \%$ RMS error for FRF magnitude as shown in Figure 5.6. It should be noted that the OSM is in excellent agreement with the maximum recovered 
load from GFEM, which are typically used for the construction of the critical loads envelop used for structural sizing and optimization. It can also be seen in Figure 5.6 and Figure 5.7 that the OSM ROM behavior deviates from that of GFEM at higher frequencies.

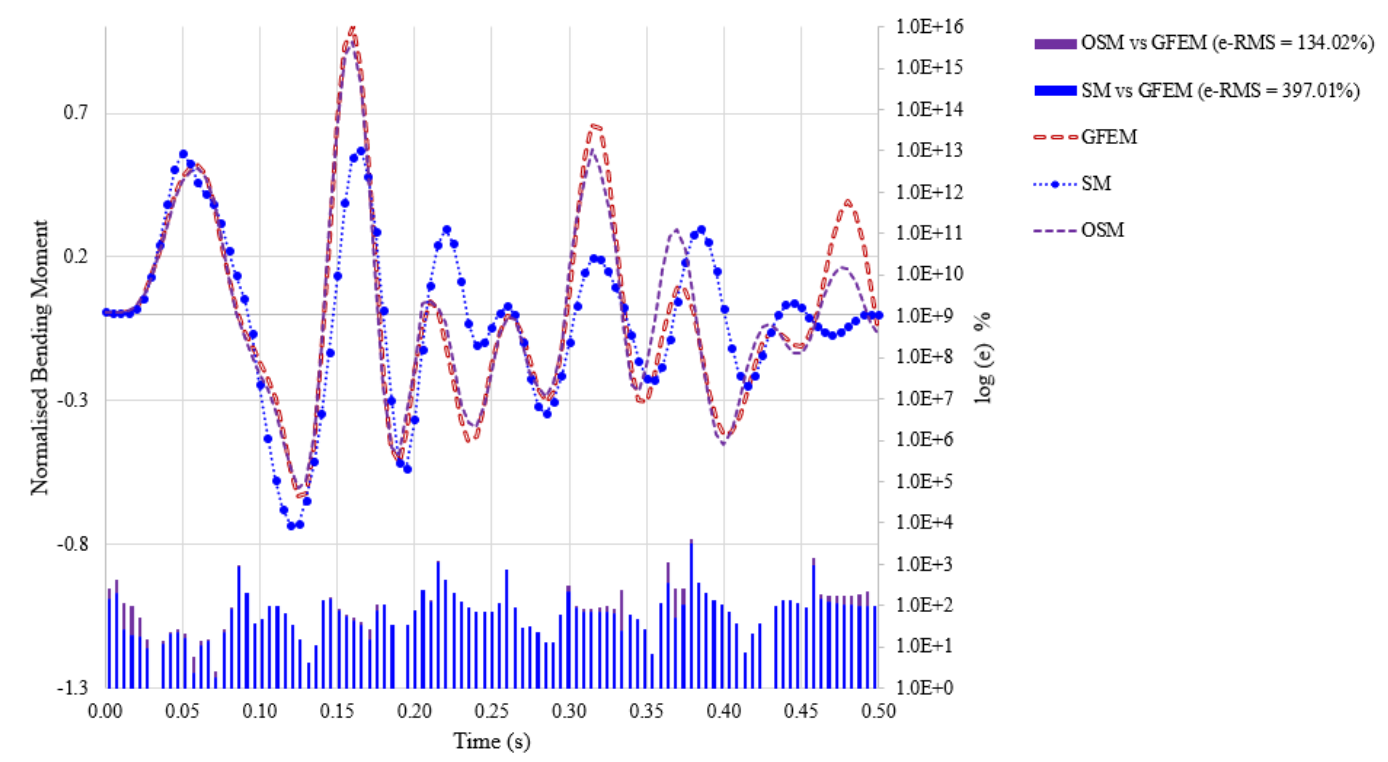

Figure 5.5 Comparison of out of plane bending moment recovered from SM, OSM and GFEM 


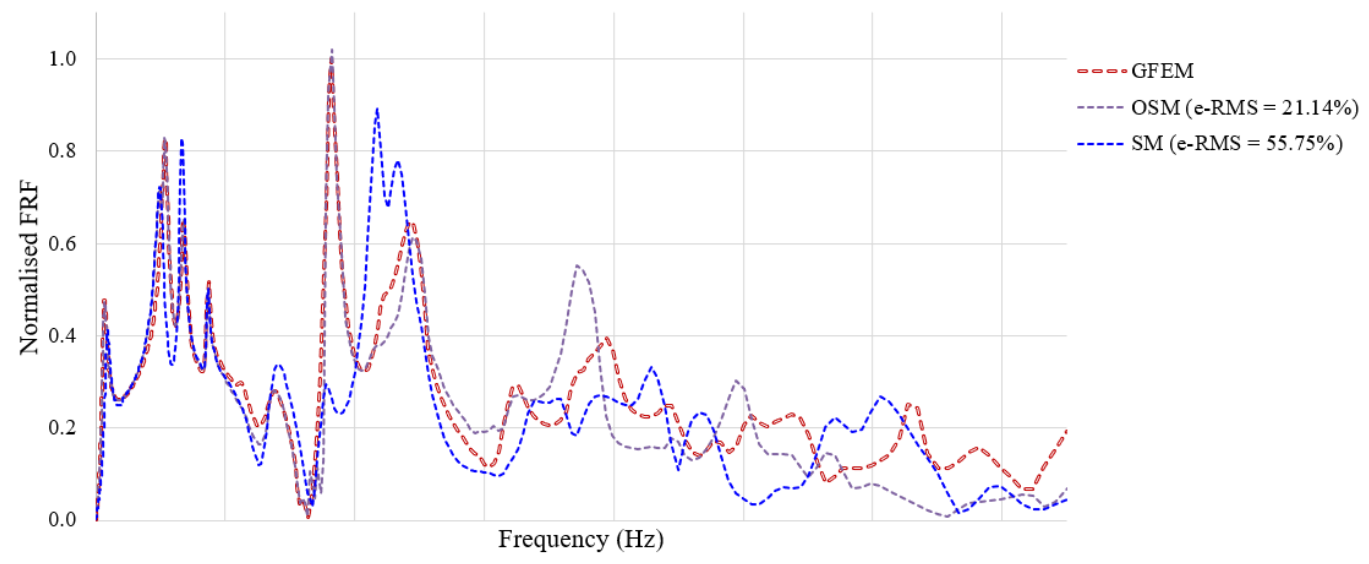

Figure 5.6 Comparison frequency response function (Magnitude) for SM, OSM and GFEM

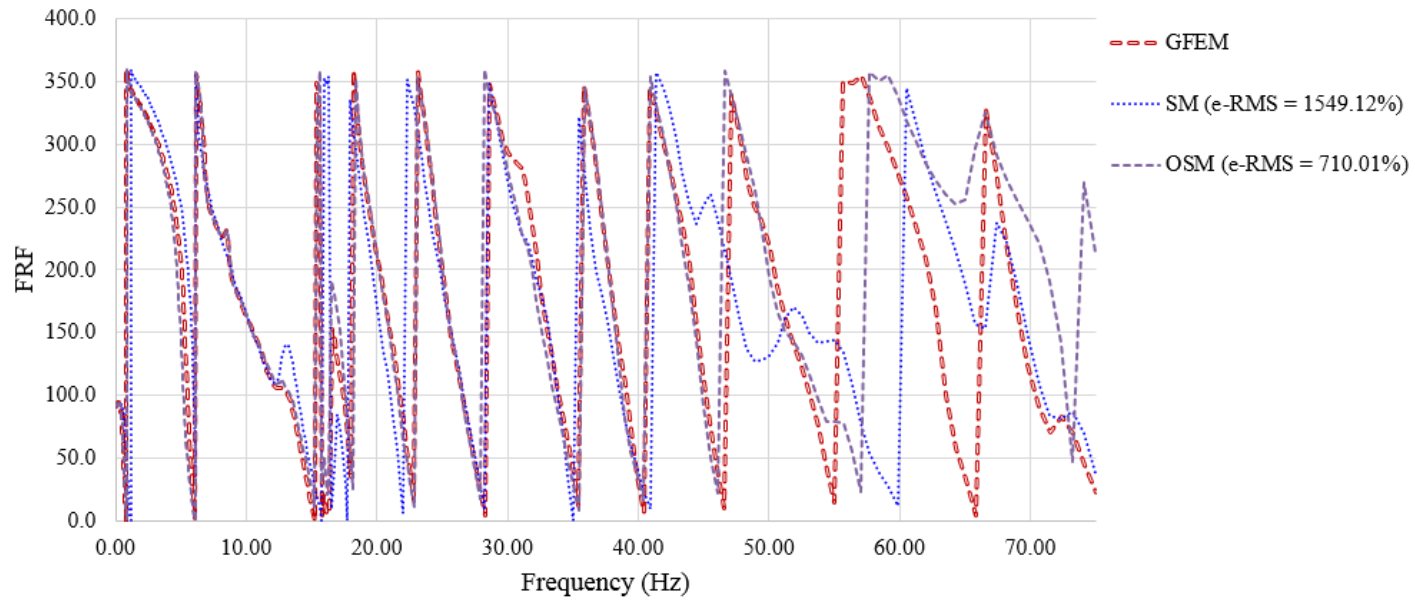

Figure 5.7 Comparison frequency response function (phase) for SM, OSM and GFEM 


\subsubsection{Modal Participation}

The MPF for all flexible modes in the frequency range of interest in the dynamic aeroelasticity response analysis (TDG case) is compared for the SM and OSM ROM in reference to the GFEM. MPF is found out at grid ID 1015 along the translational DoF, T3, at time 0.10 second as shown in Figure 5.8. The major normal modes which participate in the response analysis of the GFEM include the elastic modes 1, 2, 3 and 9. It can be seen that the OSM ROM modes participation shows the same behavior as the GFEM whereas the base SM ROM has a higher RMS error which shows its poor agreement with the GFEM.

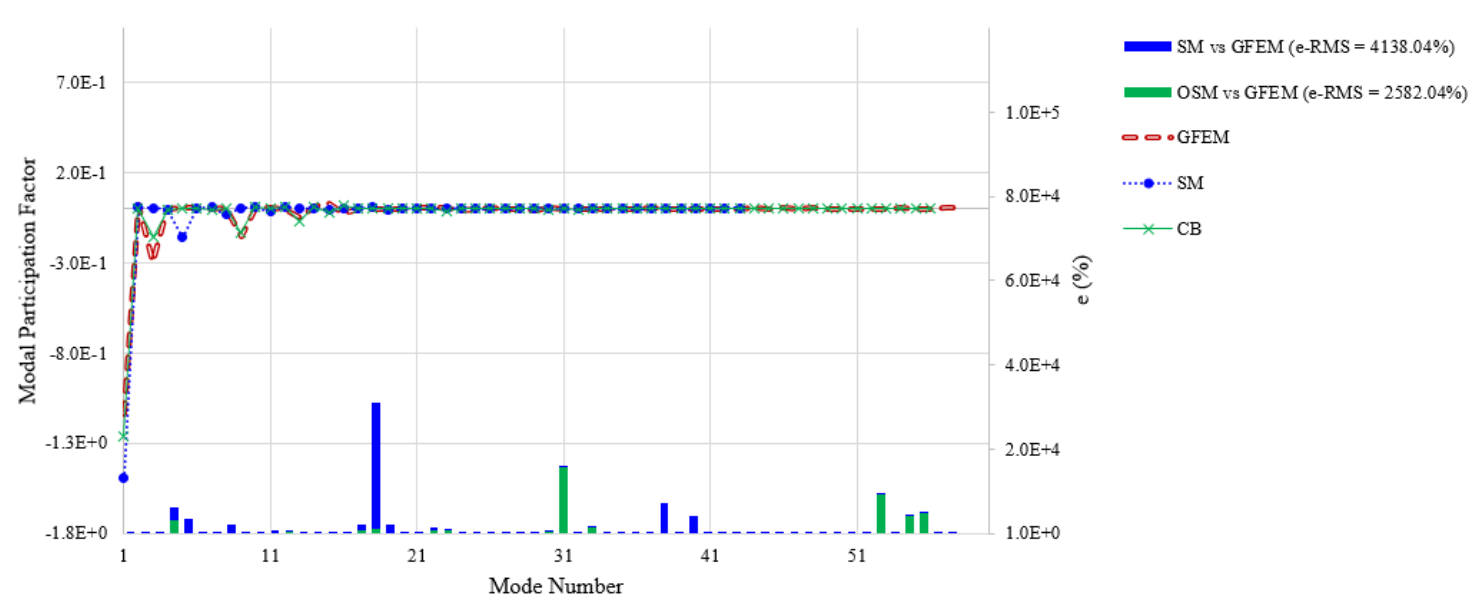

Figure 5.8 Comparison of Modal Participation Factor for SM, OSM and GFEM

The RMS error associated with the SM and the OSM ROMs based on different analysis criteria investigated in the current case study is summarized as shown in Table 5.1. It can 
be seen that the RMS error associated with the OSM ROM in all analysis criteria is much less as compared to the SM ROM.

Table 5.1 Summary of RMS error associated with SM and OSM ROMs for each analysis

\begin{tabular}{ccccc}
\hline \multirow{2}{*}{ ROM } & \multicolumn{4}{c}{ e-RMS (\%) } \\
\cline { 2 - 5 } & Normal mode & \multicolumn{3}{c}{ Dynamic Aeroelasticity } \\
\cline { 2 - 5 } & analysis & TDG & PSD (Mag) & PSD (Phase) \\
\hline SM & 12.21 & 397.01 & 55.75 & 1549.12 \\
OSM & 4.36 & 134.02 & 21.14 & 710.01 \\
\hline
\end{tabular}

The analysis cost associated with the OSM is the same as that of the base SM ROM since both these ROMs have same reduced matrix order and the same type of Timoshenko beam elements. 


\section{Conclusion and Future Work}

This thesis presents two high fidelity ROMs, namely, HSM and OSM for the dynamic aeroelasticity analysis. In addition, the applicability of eleven MOR methodologies available in the literature are reviewed with particular focus on their compatibility for aeroelasticity load recovery applications. A summary of the main features of the different MOR methods is presented in Table 6.1. All MOR methods are broadly classified based on their coordinate representation into physical, modal and hybrid ROMs. It is shown that ROMs represented in the mathematical modal coordinate are not suitable for aircraft aeroelasticity loads analysis as flight conditions are presented in the physical space. On the other hand, MOR methods such as DGP, KGP and POD are developed taking into consideration the model applied loading. Accordingly, they are deemed inefficient for the iterative aeroelasticity loads analysis process. Therefore, a total of 6 ROMs from the literature are adopted for a case study of a Bombardier aircraft platform as shown in chapter 3. Based on the results of this case study, ROMs are ranked taking into consideration their static and dynamic fidelity where the accuracy of the recovered loads in, respectively, static and dynamic aeroelasticity analyses is used as the comparison criteria. Here, the error is computed as the RMS value of difference in loads calculated using the ROMs versus those found using the GFEM of the aircraft. In addition, the normal mode analysis results showed that all matrix based ROMs have eigenvalues closely matching those of the GFEM with the least error seen in CMS ROMs. Another comparison is also performed for the different ROMs based on their computational cost in the form of time required for their development as well as their efficiency in aeroelastic solution that they are adopted for. Finally, a comparison of the ROMs based on the loads extraction process [9] that can be used for 
each model representation, namely, Deformation Approach (DA) and Momentum Approach (MA) methods, is also performed. 
Table 6.1 Comparison of ROMs based on their compatibility for aeroelastic loads recovery applications

\begin{tabular}{|c|c|c|c|c|c|c|c|c|}
\hline \multirow{3}{*}{ MOR } & \multirow{3}{*}{$\begin{array}{c}\text { Coordinate } \\
\text { Representation }\end{array}$} & \multirow{3}{*}{$\begin{array}{l}\text { Compatibility } \\
\text { with MSC } \\
\text { Nastran }\end{array}$} & \multirow{3}{*}{$\begin{array}{l}\text { Need for ROM } \\
\text { regeneration for } \\
\text { loads iterations }\end{array}$} & \multirow{3}{*}{$\begin{array}{l}\text { Fidelity in Static } \\
\text { Aeroelasticity } \\
\text { Analysis } \\
\text { 1...most accurate } \\
\text { 9...least accurate }\end{array}$} & \multicolumn{2}{|c|}{$\begin{array}{c}\text { Fidelity in Dynamic } \\
\text { Aeroelasticity } \\
\text { Analysis }\end{array}$} & \multirow{3}{*}{$\begin{array}{l}\text { Computational } \\
\text { Efficiency } \\
\text { 1...most efficient } \\
\text { 8...least efficient }\end{array}$} & \multirow{3}{*}{$\begin{array}{l}\text { Loads } \\
\text { extraction } \\
\text { method }\end{array}$} \\
\hline & & & & & \multicolumn{2}{|c|}{$\begin{array}{l}1 . . . \text { most accurate } \\
9 . . . \text { least accurate }\end{array}$} & & \\
\hline & & & & & TDG & PSD & & \\
\hline SM & Physical & YES & $\mathrm{NO}$ & 9 & 9 & 9 & 1 & $\mathrm{DA}, \mathrm{MA}$ \\
\hline GR & Physical & YES & $\mathrm{NO}$ & 4 & 5 & 4 & 2 & MA \\
\hline IRS & Physical & YES & NO & 7 & 7 & 7 & 3 & MA \\
\hline DY & Physical & YES & $\mathrm{NO}$ & 5 & 4 & 6 & 4 & MA \\
\hline DGP & Physical & NO & YES & - & - & - & - & MA \\
\hline KGP & Physical & NO & YES & - & - & - & - & MA \\
\hline RMD & Modal & NO & $\mathrm{NO}$ & - & - & - & - & MA \\
\hline CMD & Modal & NO & $\mathrm{NO}$ & - & - & - & - & MA \\
\hline POD & Modal & NO & YES & - & - & - & - & MA \\
\hline $\mathrm{CB}$ & Hybrid & YES & $\mathrm{NO}$ & 1 & 3 & 2 & 6 & MA \\
\hline $\mathrm{FF}$ & Hybrid & YES & $\mathrm{NO}$ & 2 & 2 & 3 & 5 & MA \\
\hline HGR & Physical & YES & $\mathrm{NO}$ & 6 & 6 & 5 & 7 & MA \\
\hline $\mathrm{HCB}$ & Hybrid & YES & $\mathrm{NO}$ & 3 & 1 & 1 & 8 & MA \\
\hline OSM & Physical & YES & $\mathrm{NO}$ & 8 & 8 & 8 & 1 & DA, MA \\
\hline
\end{tabular}


Based on the results of the case study presented in chapter 3, among all ROMs investigated, it is found that $\mathrm{CB}$ and GR ROMs have the most dynamic fidelity as compared to the GFEM. Accordingly, these two methodologies in junction with the conventional SM, generated by unitary loading method, are employed to develop the HSM as shown in chapter 4. It is seen that the HSM ROMs have high dynamic accuracy similar to those of the matrix based reduction methods as well as having the same handling flexibility of the SM. The normal modes analysis showed that both HSMs, developed, have eigenvalues closely matching those of the GFEM. It is seen in the dynamic aeroelasticity analysis that the loads extracted from the HSMs are closely matching those of the GFEM almost everywhere in the frequency range of interest. Both HCB and HGR ROMs are ranked in Table 6.1 based on the static and dynamic fidelity of the loads recovered.

The errors seen in SM normal mode analysis and dynamic aeroelasticity loads analysis clearly indicate that the base SM is dynamically inequivalent to the GFEM. An optimization problem is performed to optimize the dynamic performance of the base SM. It is shown that the generated OSM has an improved dynamic behavior as compared to its base SM. The normal modes analysis showed a reduction in e-RMS from $12.11 \%$ for base SM to $4.36 \%$ for the OSM. In addition, the SM design optimization significantly improved the mode shapes as we showed that the effectively participating 10 elastic modes are in good agreement with the corresponding modes of the GFEM. We also showed that the OSM ROM is in excellent agreement with the GFEM maximum loads recovered from dynamic aeroelasticity analysis in TDG and PSD cases. Those maximum loads are essentially used to construct the critical loads envelope rendering the OSM very efficient for loads recovery applications. 
Finally, a MPF study conducted in the dynamic aeroelastic response analyses showed that the dominant modes of HSM and OSM ROMs are exactly matching those of the GFEM. Moreover, HSMs and OSM are computationally cheap as compared to the GFEM. Hence, the two models developed in this thesis serve as excellent candidates for aeroelasticity load analyses with dynamic characteristics closely matching those of the GFEM with low analysis cost.

\subsection{Future Work}

It is found that computational cost to develop the OSM is high due to the metaheuristic type optimization algorithm used. The difficulty of finding the optimal solution increases exponentially with the number of design variables representing the SM beams stiffness parameters. Looking into other metaheuristic algorithm such as enhanced Scatter Search [112], to accelerate the convergence is an interesting point for future research. Also, the higher generation cost associated with the current stick optimization can be lowered by providing appropriate constraint which indicate the approximate stiffness distribution along different aircraft bays in the GFEM. An approximate beam element stiffness distribution at each wing station can be interpreted from the diagonal values of the reduced Guyan stiffness matrix, which is a diagonally dominated matrix.

In addition, as design optimization changes the static and dynamic characteristics of the GFEM, it is of particular interest to develop an efficient tool for the regeneration of the ROM for every change in the structural parameters of the GFEM such as skin thickness, wing root chord, tip chord, sweep angle etc. Here, we propose a methodology to appropriately map ROM data into a tangent space to the manifold of symmetric positive 
definite matrices such as Mass, Stiffness and Damping. Then, interpolating the mapped data in tangent space and mapping back the result to the physical GFEM space [111]. This procedure can be applied to ROM based on Galerkin projection. Hence, this interpolation method to map the HSM ROM data to GFEM and backward, can be implemented in future. 


\section{A Appendix: Timoshenko Beam element stiffness matrix}

Consider a beam finite element having two nodes and 6 DoFs at each node. The

displacement vector and stiffness matrix for such an element, assuming no warping considered at this stage, as shown in Eq. (A.1) and (A.2) respectively. These beam elements are expressed with respect to the elastic axis as shown in Figure A.1

$$
\begin{aligned}
& x=\left\{\begin{array}{llllllllllll}
u_{C 1} & v_{C 1} & w_{C 1} & \theta_{X 1} & \theta_{Y 1} & \theta_{Z 1} & u_{C 2} & v_{C 2} & w_{C 2} & \theta_{X 2} & \theta_{Y 2} & \theta_{Z 2}
\end{array}\right\} \\
& K_{c}=\left[\begin{array}{cccc}
K_{1} & K_{1} Q_{1} & -K_{1} & -K_{1} Q_{2} \\
\cdots \cdots \cdots \cdots \cdots \cdots & Q_{1}{ }^{T} K_{1} Q_{1}+K_{2} & -Q_{1}{ }^{T} K_{1} & -Q_{1}{ }^{T} K_{1} Q_{2}-K_{2} \\
\cdots \cdots \cdots \cdots \cdots \cdots & \cdots \cdots \cdots \cdots \cdots \cdots \cdots & K_{1} & K_{1} Q_{2} \\
\text { symmetric } & \cdots \cdots \cdots \cdots \cdots \cdots \cdots & \cdots \cdots \cdots \cdots & Q_{2}{ }^{T} K_{1} Q_{2}+K_{2}
\end{array}\right]
\end{aligned}
$$

where the base matrices $K_{1}$ and $K_{2}$ are defined by,

$$
K_{1}=\left[\begin{array}{ccc}
\frac{E A}{L} & 0 & 0 \\
0 & \frac{12 E I_{z}}{\left(1+\phi_{y}\right) L^{3}} & 0 \\
0 & 0 & \frac{12 E I_{y}}{\left(1+\phi_{z}\right) L^{3}}
\end{array}\right]
$$

where $\phi_{y}=12 E I_{z} / k_{y} A G L^{2}$ and $\phi_{z}=12 E I_{y} / k_{z} A G L^{2}$ corresponds to the shear effects corrections. 


$$
K_{2}=\left[\begin{array}{ccc}
\frac{G J}{L} & 0 & 0 \\
0 & \frac{E I_{y}}{L} & 0 \\
0 & 0 & \frac{E I_{z}}{L}
\end{array}\right]
$$

The matrices $Q_{1}$ and $Q_{2}$ depend only on the co-ordinates of $\mathrm{C}$ and $\mathrm{G}$ expressed with respect to the principal frame centered at $\mathrm{O}$. It leads to,

$$
\begin{aligned}
Q_{1} & =\left[\begin{array}{ccc}
0 & z_{G} & -y_{G} \\
-z_{C} & 0 & L / 2 \\
y_{C} & -L / 2 & 0
\end{array}\right] \\
Q_{2} & =\left[\begin{array}{ccc}
0 & z_{G} & -y_{G} \\
-z_{C} & 0 & -L / 2 \\
y_{C} & L / 2 & 0
\end{array}\right]
\end{aligned}
$$

where $y_{G}=y_{C}+\overline{y_{G}}$ and $z_{G}=z_{C}+\overline{z_{G}}$. Refer to Figure A.2 for the distance along $\mathrm{y}$ and $\mathrm{z}$ from the origin to the CG and shear center.

The beam element stiffness matrix with coincident mass and shear center can be formulated from (A.2) by substituting zero offset distance (i.e., $y_{G}, z_{G}, y_{c}, z_{c}=0$ ). 


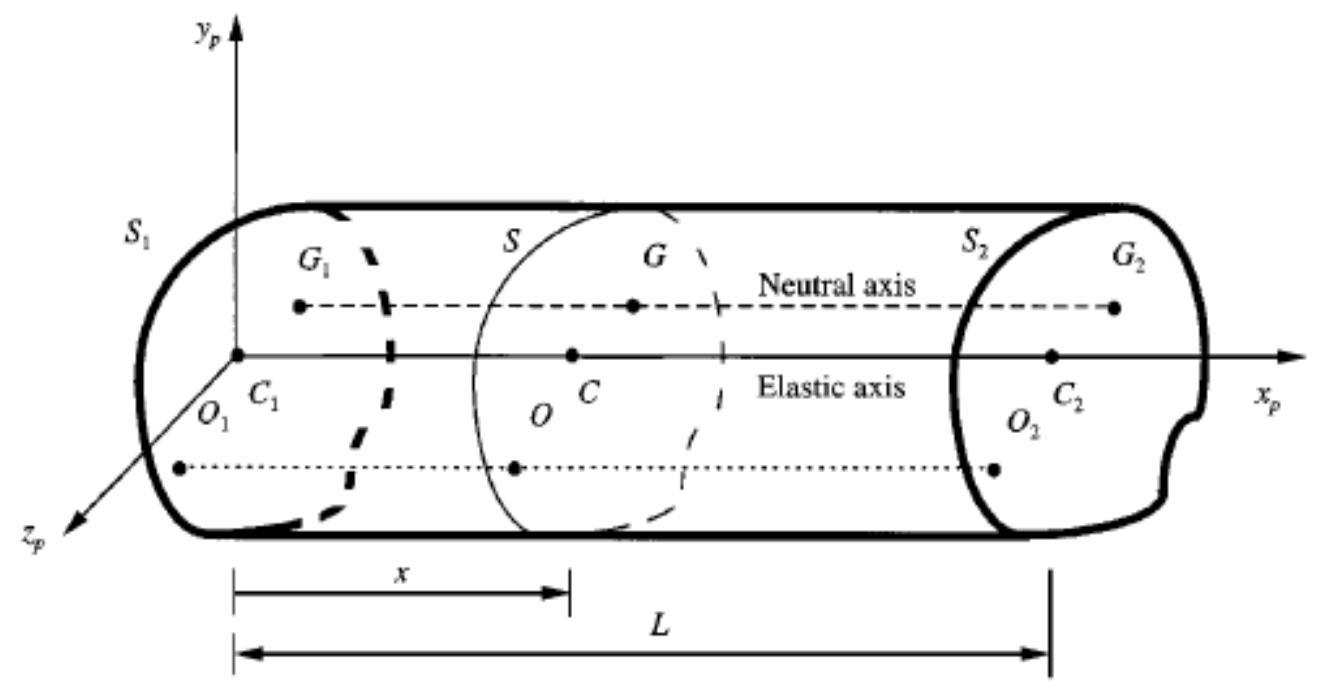

Figure A.1 Straight beam with uniform cross section

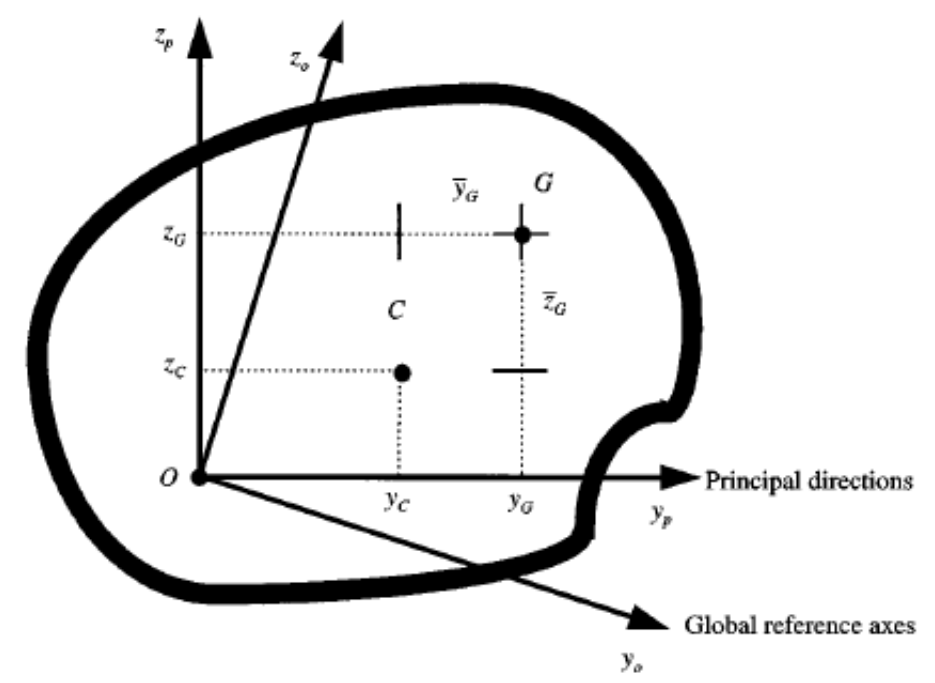

Figure A.2 Location of parameters in a cross section

In order to form the most general formulation for the equivalent beam, the elementary stiffness matrix have to be expressed in an arbitrary direction of the global computational reference frame $\left(z_{o}, y_{o}\right)$. The rotation matrix $R$ defining the principal axes with respect to global reference frame is given by, 


$$
R=\left[\begin{array}{llll}
\Theta & 0 & 0 & 0 \\
0 & \Theta & 0 & 0 \\
0 & 0 & \Theta & 0 \\
0 & 0 & 0 & \Theta
\end{array}\right], \Theta^{T} \Theta=\mathrm{I}
$$

where $\Theta$ include the directional cosines of principal axes $\left(x_{p}, y_{p}, z_{p}\right)$ with respect to the global reference axes orientation $\left(x_{o}, y_{o}, z_{o}\right)$.

$$
\left[K_{o}\right]_{E}=[R]^{T}\left[K_{c}\right][R]
$$

All the parameters of the analytical beam element is defined with respect to the $\left(O_{1}, O_{2}\right)$ point and arbitrary axes $\left(x_{o}, y_{o}, z_{o}\right)$. 


\section{REFERENCES}

1 Wright, J. and J. Cooper, Introduction to Aircraft Aeroelasticity and Loads, McGraw Hill, 2007.

2 Howe D, Aircraft Loading and Structural layout, AIAA Education Series, 2004.

3 Federal Aviation Administration, Airworthiness Standards: Transport Category Airplanes, FAR-25.

4 Europa Aviation Safety Agency, Certification Specifications for Large Aeroplanes, CS25, Amendment 3, 2007.

5 Frederic M. Hoblit, "Gust Loads on Aircraft: Concepts and Applications", AIAA Education Series, 1988.

6 MG Nieto, MSA ElSayed, D Walch, Improved Modal Contribution Factors as Response Tracking Mechanisms for Dynamic Systems During Design Optimization, CSME International Congress 2018.

7 P. V. Thomas, M. S. A. ElSayed and Denis Walch, "Review of Model Order Reduction Methods and their Applications in Aeroelasticity Loads Analysis for Design Optimization of Complex Airframes”, ASCE, Journal of Aerospace Engineering, 2018.

8 P. V. Thomas, M. S. A. ElSayed and Denis Walch, "Development of High Fidelity Reduced Order Hybrid Stick Model for Aircraft Dynamic Aeroelasticity Analysis”, Journal of Aerospace Science and Technology, Ref. AESCTE_2018_1605.

9 M.S.A. ElSayed, M.G. Contreras, and N. Stathopoulos, "Monitor Points Method for Loads Recovery in Static/Dynamic Aeroelasticity Analysis with Hybrid Airframe Representation,” SAE Int. J. Aerosp. vol. 6, no. 2, 2013. 
10 M.S.A. ElSayed, R. Sedaghati, and M. Abdo, “Accurate Stick Model Development for Static Analysis of Complex Aircraft Wing-Box Structures," AIAA JOURNAL, vol. 47, no. 9, September. 2009.

11 G. Bindolino, G. Ghiringhelli, S. Ricci, and M. Terraneo, "Multilevel structural optimization for preliminary wing-box weight estimation,” Journal of Aircraft, vol. 47, no. 2, pp. 475-489, March-April. 2010.

12 A. K. Singh and C. W. Nichols, "Derivation of an equivalent beam model from a structural finite element model," in Proceedings of the MSC 1988 World Users Conference, Los Angeles, Calif, USA,1988.

13 G. Corriveau and F. Dervault, "Impact of wing-box geometrical parameters on stick model prediction accuracy," 54th AIAA/ASME/ASCE/AHS/ASC Structures, Structural Dynamics, and Materials Conference, Boston, Massachusetts, USA, April 811, 2013.

14 Hashemi-Kia .M and Toossi .M, "Development and application of a technique for reducing airframe finite element models for dynamic analysis," NASA-CR-187448, NAS 1.26:187448, McDonnell-Douglas Helicopter Co., Mesa, AZ, USA, October 1990.

15 R. Cirillo, "Detailed and condensed finite element models for dynamic analysis of a business jet aircraft, MSc thesis, The University of Politecnico di Milano, Italy, 20102011.

16 MSC Software Corporation, MSC PATRAN User's Guide, 2016.

17 Paul Kuhn, National Advisory Committee for Aeronautics. Technical notes No. 562. Remarks on the elastic axis of shell wings. Langley Memorial Aeronautics Laboratory. Washington. April 1936. 
18 R. J. Guyan, "Reduction of stiffness and mass matrices," AIAA Journal, vol. 3, no. 2, pp. 380-380, 1965. https://doi.org/10.2514/3.2874.

19 Irons, B., "Structural eigenvalue problems-elimination of unwanted variables," AIAA Journal, vol. 3, no. 5, pp. 961-962, 1965.

20 R.L. Kidder, "Reduction of structural frequency equations," AIAA journal, vol. 11, no. 6, p. 892, 1973.

21 E.J. Kuhar and C.V. Stahle, "Dynamic transformation method for modal synthesis," AIAA journal, vol. 12, no. 5, pp. 672-678, 1974.

22 O'Callahan, J. C., "A procedure for an improved reduced system (IRS) model," in Proceedings of the 7th international modal analysis conference, Union College Press, Schenectady, NY, Vol. 1, pp.17-21, February 1989.

23 Gordis, J. H., "An analysis of the improved reduced system (IRS) model reduction procedure," in 10th International Modal Analysis Conference, vol. 1, pp. 471-479, 1992.

24 Leung, A. Y. T., "An accurate method of dynamic condensation in structural analysis. International Journal for Numerical Methods in Engineering, vol. 12, no. 11, pp. 1705$1715,1978$.

25 Bampton, M. C., and Craig, Jr, R. R., "Coupling of substructures for dynamic analyses," AIAA Journal, vol. 6, no. 7, pp. 1313-1319, 1968.

26 R.M. Bamford, "A Modal Combination program for Dynamic Analysis of Structures," Technical Memorandum 33-290, Jet Propulsion Laboratory, Pasadena CA, Revision No. 1, July 1, 1967. 
27 Benfield, W. A., and Hurda, R. F., "Vibration Analysis of Structures by Component Mode Substitution," AIAA Journal, vol. 9, no. 7, pp. 1255-1261, July 1971. https://doi.org/10.2514/3.49936.

28 Rubin, S., "Improved Component-Mode Representation for Structural Dynamic Analysis," AIAA Journal, vol. 13, no. 8, pp. 995-1006, August 1975. https://doi.org/10.2514/3.60497.

29 Bennighof, J. K., and Lehoucq, R. B., "An Automated Multilevel Substructuring Method for Eigenspace Computation in Linear Elastodynamics", SIAM Journal on Scientific Computing, vol. 25, no. 6, pp. 2084-2106, 2004. https://doi.org/10.1137/S1064827502400650.

30 MacNeal, R. H., "A Hybrid Method of Component Mode Synthesis," Computers and Structures, vol. 1, no. 4, pp. 581-601, December 1971. https://doi.org/10.1016/00457949(71)90031-9.

31 Park, K. C., and Park, Y. H., "Partitioned Component Mode Synthesis via a Flexibility Approach", AIAA Journal, vol. 42, no. 6, pp. 1236-1245, June 2004. https://doi.org/10.2514/1.10423.

32 Rixen, D. J., "A Dual Craig-Bampton Method for Dynamic Substructuring," Journal of Computational and Applied Mathematics, vol. 168, no. 1-2, pp. 383-391, July 2004. https://doi.org/10.1016/j.cam.2003.12.014.

33 Klerk, D. D., Rixen, D. J., and Voormeeren, S. N., "General Framework for Dynamic Substructuring: History, Review and Classification of Techniques," AIAA Journal, vol. 46, no. 5, pp. 1169-1181, May 2008. https://doi.org/10.2514/1.33274. 
34 Goldman, R L., "Vibration Analysis by Dynamic Partitioning," AIAA Journal, vol. 7, no. 6, pp. 1152-1154, 1969. https://doi.org/10.2514/3.5290.

35 Beattie C, and Gugercin S., "Interpolatory projection methods for structure-preserving model reduction," Systems and Control Letters, vol. 58, no. 3, pp. 225-232, march 2009. https://doi.org/10.1016/j.sysconle.2008.10.016.

36 Djellouli R, Farhat C, Tezaur R., "A fast method for solving acoustic scattering problems in frequency bands," Journal of Computational Physics, vol. 168, no. 2, pp. 412-432, April 2001. https://doi.org/10.1006/jcph.2001.6707.

37 Avery, P., Farhat, C. and Reese, G., "Fast frequency sweep computations using a multipoint Padé-based reconstruction method and an efficient iterative solver," International Journal for Numerical Methods in Engineering, 69: pp. 2848-2875, 2006. doi: 10.1002/nme. 1879 .

38 Lecomte C, McDaniel JG, and Barbone P. "Two error bounds for dynamic condensation," AIAA Journal; 46, no. 1, pp. 166-176, 2008. https://doi.org/10.2514/1.29866.

39 Liew HL, Pinsky P. "Matrix-Padé via Lanczos solutions for vibrations of fluid-structure interaction," International Journal for Numerical Methods in Engineering; vol. 84, no. 10, pp. 1183-1204, November 2010. doi: 10.1002/nme.2936.

40 Meerbergen K., "Fast frequency response computation for Rayleigh damping," International Journal for Numerical Methods in Engineering, vol. 73, no. 1, pp. 96-106, doi: $10.1002 / n m e .2058$.

41 Olsson K., "Model order reduction with rational Krylov methods," PhD thesis, KTH, Sweden, September 2005. 
42 Tuck-Lee J, and Pinsky P. "Adaptive frequency windowing for multifrequency solutions in structural acoustics based on the matrix Padé-via-Lanczos algorithm," International Journal for Numerical Methods in Engineering, vol. 73, no. 5, pp. 728746, 2008. doi: 10.1002/nme.2102.

43 Wagner M, Pinsky P, Oberai A, and Malhotra M. A, "Krylov subspace projection method for simultaneous solution of Helmholtz problems at multiple frequencies," Computer Methods in Applied Mechanics and Engineering; 192:4609-4640, 2003. https://doi.org/10.1016/S0045-7825(03)00429-8.

44 Beattie C, and Gugercin S., "Krylov-based model reduction of second-order systems with proportional damping," Proceedings of 44th CDC/ECC, Seville, Spain, pp: 22782283, 2003.

45 P.Seshu, "Review on substructuring and component mode synthesis," John Wiley \& Sons, Inc, Vol. 4, pp 199-210, 1997.

46 Hou, S., "Review of Modal Synthesis Techniques and New Approach," Shock and Vibration Bulletin, Vol. 40, pp. 25-39, 1969.

47 Young, J. T., and Haile W. B. "Primer on the Craig-Bampton method. Finite Element Modeling Continuous Improvement”, 2000.

48 Sanchez, R.R., Buchschmid, M., and Muller, G., "Model Order Reduction in structural dynamics," in ECCOMAS Congress, 2016.

49 Hetmaniuk, U., Tezaur, R., and Farhat, C., "Review and assessment of interpolatory model order reduction methods for frequency response structural dynamics and acoustics problems," International Journal for Numerical Methods in Engineering, vol. 90, no. 13, pp. 1636-1662, 2012.doi: 10.1002/nme.4271. 
50 Strang, G., "Introduction to linear algebra". Wellesley, MA: Wellesley-Cambridge Press, Vol.3, 1993.

51 Antoulas A., "Approximation of Large-Scale Dynamical Systems," SIAM: Philadelphia, pp. 343-361, 2005.

52 Bai Z, Dewilde P, and Freund R., "Reduced-order modeling," In Numerical Methods in Electromagnetics, volume XIII of Handbook of Numerical Analysis, Ciarlet P (ed), Elsevier, 2005.

53 Freund, R., "Model reduction methods based on Krylov subspaces," Acta Numerica, 12, pp. 267-319, 2003. doi: 10.1017/S0962492902000120.

54 M.I. Friswell, S.D. Garvey, and J.E.T. Penny, "The convergence of the iterated IRS method," Journal Sound Vibration, vol. 211, no. 1, pp. 123-132, 1998. https://doi.org/10.1006/jsvi.1997.1368.

55 L.E. Suarez, "Dynamic condensation method for structural eigenvalue analysis", AIAA journal, vol. 30, no. 4, 1992, pp. 1046-1054.

56 M.I. Friswell, S.D. Garvey, J.E.T. Penny, "Model reduction using dynamic and iterated IRS techniques", Journal of Sound and Vibration, vol. 186, no. 2, pp. 311-323, 1995.

57 K.O. Kim, and M.K. Kang, "Convergence acceleration of iterative modal reduction methods," AIAA journal, vol. 39, no. 1, pp. 134-140, 2001.

58 D. Choi, H. Kim, and M. Cho, "Iterative method for dynamic condensation combined with substructuring scheme," Journal of Sound and Vibration, vol. 317, no. 1-2, pp. 199-218, October 2008. 
59 M.I Friswell, S D Garvey and J E T Penny, "Using iterated IRS model reduction techniques to calculate eigensolutions," Proceedings of SPIE - The International Society for Optical Engineering 2, January 1997.

60 Y. Xia and R. M. Lin, "Improvement on the iterated IRS method for structural eigensolutions," Journal of Sound and Vibration, Volume 270, Issues 4-5, Pages 713727, March 2004.

61 Xia, Y., Lin, R.M. “A new iterative order reduction (IOR) method for eigensolutions of large structures," International Journal for Numerical Methods in Engineering, vol. 59, no. 1, pp. 153-172, 2004.

62 Liu, Z.S., and Wu, Z.G., "Iterative-order-reduction substructuring method for dynamic condensation of finite element models," AIAA Journal, vol. 49, no. 1, pp. 87-96, 2001.

63 Weng, S., Xia, Y., Xu, Y.L., Zhu, H. P., "An iterative substructuring approach to the calculation of eigensolution and eigensensitivity," Journal of Sound and Vibration. Vol. 330, no. 14, pp. 3368-3380, 2011.

64 Philip Avery, Charbel Farhat and Garth Reese, "Fast frequency sweep computations using a multi-point Pad'e-based reconstruction method and an efficient iterative solver" Wiley InterScience, DOI: 10.1002/nme.1879, pp 2848-2875, published online 5 September 2006.

65 Computational Methods in Structural Dynamics, Meirovitch. L, 1980.

66 Homes, P., Lumley J, Berkooz G. (1996). “Turbulence, Coherent Structures, Dynamical Systems and Symmetry.” Cambridge University Press. 
67 Kerschen, G., Golinval, J. C., Vakakis, A. F., and Bergman, L. A. (2005). “The method of proper orthogonal decomposition for dynamical characterization and order reduction of mechanical systems: an overview.” Nonlinear dynamics, 41(1-3), 147-169.

68 Amabili, M., Sarkar, A., and Paidoussis, M. P. (2003). "Reduced-order models for nonlinear vibrations of cylindrical shells via the proper orthogonal decomposition method.” Journal of Fluids and Structures, 18(2), 227-250.

69 Han, S., \& Feeny, B. F. (2002). "Enhanced proper orthogonal decomposition for the modal analysis of homogeneous structures.” Modal Analysis, 8(1), 19-40.

70 Hurty W., "Dynamic analysis of structural systems using component modes," AIAA Journal, vol. 3, no. 4, pp. 678-685, 1965.

71 Kim, J. G., Boo, S. H., and Lee, P. S., "An Enhanced AMLS Method and Its Performance", Computer Methods in Applied Mechanics and Engineering, Vol. 287, April 2015, pp. 90-111, doi 10.1016/j.cma.2015.01.004.

72 Kim, J. G., Lee, P. S., "An Enhanced Craig-Bampton Method", International Journal for Numerical Methods in Engineering, Vol. 103, pp. 79-93, March 2015. doi 10.1002/nme.4880.

73 Kim, J. G., Boo, S. H., Lee, P. S., "Performance of the Enhanced Craig-Bampton Method", Structural Engineering \& Mechanics (ASEM2015), 2015.

74 A. Ibrahimbegovic, and E. L. Wilson, "Automated truncation of Ritz vector basis in modal transformation," ASCE Journal of Engineering Mechanics, vol. 116, no. 11, pp. 2506-2520, 1990. 
75 D. Markovic, K.C. Park, and A. Ibrahimbegovic, "Reduction of substructural interface degrees of freedom in flexibility-based component mode synthesis," International Journal for Numerical Methods in Engineering, vol. 70, pp. 163-180, 2007.

76 K.C. Park, J.G. Kim, and P.S. Lee, "A mode selection criterion based on flexibility approach in component mode synthesis," Proceeding 53th AIAA/ASME/ASCE/AHS /ASC Structures, Structural Dynamics, and Materials Conference USA, Hawaii, 2012.

77 Cui J., Xing J., Wang X., Wang Y., Zhu S., and Zheng G., “A Simultaneous Iterative Scheme for the Craig-Bampton Reduction Based Substructuring," Dynamics of Coupled Structures, vol.4, Conference Proceedings of the Society for Experimental Mechanics Series, Springer, 2017. https://doi.org/10.1007/978-3-319-54930-9_10.

78 MSC Software Corporation, “MSC NASTRAN 2016 Quick Reference Guide,” April 26, 2016.

79 R.D. Henshell, and J.H. Ong, "Automatic masters from eigenvalues economization", Earthquake Engineering Structural Dynamics, vol. 3, pp. 375-383, 1975.

80 J.H. Ong, "Improved automatic masters for eigenvalues economization", Finite Element Analysis and Design, vol. 3 no. 2, pp. 149-160, 1987.

81 V.N. Shah, and M. Raymund, "Analytical selection of masters for the reduced eigenvalue problem," International Journal for Numerical Methods in Engineering, vol. 18, no. 1, pp. 89-98, 1982.

82 K.O. Kim, and Y.J. Choi, "Energy method for selection of degrees of freedom in condensation," AIAA Journal, vol. 38, no. 7, pp. 1253-1259, 2000. https://doi.org/10.2514/2.1095. 
83 M. Cho, and H. Kim, "Element-based node selection method for reduction of eigenvalue problems," AIAA Journal, vol. 42, no. 8, pp. 1677-1684, 2004.

84 Blair, M. A., Camino, T. S., and Dickens, J. M., "An iterative approach to a reduced mass matrix," in 9th Conference International Modal Analysis Conference (IMAC), vol. 1, pp. 621-626, 1991.

85 Flanigan, C., and Christopher, "Model reduction using Guyan, IRS, and dynamic methods," in Proceeding of 16th International Modal Analysis Conference, pp. 172176, 1998.

86 T. J., Su and R. R. Craig, Jr., "Model reduction and control of flexible structures using Krylov vectors,” Journal Guidance Control Dynamic., vol. 14, pp. 260-267, 1991.

87 Craig, R. R., and Kurdila, A. J., "Fundamentals of structural dynamics. John Wiley \& Sons, 2006.

88 Meirovitch, L., "Fundamentals of vibrations", Waveland Press, 2010.

89 Huang, H. H., and Craig, R. R., Jr., "System Identification and Model Updating for Substructures," Report CAR 92-1, Center for Aeromechanics Research, The University of Texas at Austin, April 1992.

90 Kammer, D. C., and Triller, M. J., "Ranking the Dynamic Importance of Fixed Interface Modes Using a Generalization of Effective Mass", Modal Analysis: The International Journal of Analytical and Experimental Modal Analysis, vol. 9, no. 2, pp. 77-98, 1994.

91 Kammer, D. C., Triller, M. J., "Selection of Component Modes for Craig-Bampton Substructure Representations", 36th Structures, Structural Dynamics and Materials Conference, LA, U.S.A, 1995. 
92 Barbone, P. E., Givoli, D., and Patlashenko, I., "Optimal Modal Reduction of Vibrating Substructures", International Journal for Numerical Methods in Engineering, vol. 57, no. 3, pp. 341-369, March 2003.

93 Givoli, D., Barbone, P. E., Patlashenko, I., "Which Are the Important Modes of a Subsystem?", International Journal for Numerical Methods in Engineering, vol. 59, no. 2, pp. 1657-1678, March 2004.

94 Liao, B. S., Bai, Z., and Gao, W., "The Important Modes of Subsystems: A MomentMatching Approach," International Journal for Numerical Methods in Engineering, vol. 70, no. 13, pp. 1581-1597, June 2007.

95 Dowell, E. H., "Free Vibrations of an Arbitrary Structure in Terms of Component Modes," Journal of Applied Mechanics, vol. 39, no. 3, pp. 727-732, September 1972.

96 Yee, E. K. L., and Tsuei, Y. G., "Direct Component Modal Synthesis Technique for System Dynamic Analysis," AIAA Journal, Vol. 27, No. 8, August 1989, pp.1083-1088.

97 W.A. Benfield, C. S. Bodley and G. Morosow, "Modal synthesis methods", presented at the space shuttle dynamics and aeroelasticity working group symposium on substructure testing and synthesis, NASA-TM-X-73218, Marshall space flight center, AL, 1972.

98 U. Hayirli, A. Kayran, "Stick model development of aircraft structures for dynamic analysis", 58th AIAA/ASCE/AHS/ASC Structures, Structural Dynamics, and Materials Conference, Grapevine, Texas, 9 - 13 January. 2017.

99 E. Bosco, J. Morlier, J. Barron and A. Lucchetti "Influence of mass modelling in dynamic landing simulations" 10th PEGASUS-AIAA student conference, June 2014, Czech Republic. 
100 MSC Software Corporation, "MSC NASTRAN 2016 Quick Reference Guide,” April 26, 2016.

101 Randall J. Allemang, "The Modal Assurance Criterion - Twenty Years of Use and Abuse", sound and vibration, 2003.

102 Weaver Jr, William, Stephen P. Timoshenko, and Donovan Harold Young. "Vibration problems in engineering". John Wiley \& Sons, 1990.

103 Jrad, Mohamed, Wei Zhao, Rakesh K. Kapania, and David Schmidt. "Elastic Axis Determination and Extraction of Vibration Mode Shapes of a Light Weight Composite Aircraft." In 2018 Multidisciplinary Analysis and Optimization Conference, p. 3429. 2018.

104 MSC Nastran, Superelements User's Guide, April, 2016.

105 MSC NASTRAN, Aeroelastic Analysis User's Guide version 68, 2014.

106 P. Chudy, "Response of a light aircraft under gust loads." Acta Polytencnica, vol. 44, no. 2, 2004.

107 Ünay, E., Kiper, T., Işıkdoğan, Ö., Gürak, D., Methods for Evaluation of Aircraft Mass Distribution, 28th International Congress of the Aeronautical Sciences, Brisbane, Australia, 2012.

108 MG Nieto, MSA ElSayed, D Walch, Modal Participation Factors and their Potential Applications in Aerospace: a Review, CSME International Congress 2018.

109 Weaver Jr, William, Stephen P. Timoshenko, and Donovan Harold Young. "Vibration problems in engineering”. John Wiley \& Sons, 1990. 
110 Storn, R and Price, K, "Differential Evolution - a Simple and Efficient Heuristic for Global Optimization over Continuous Spaces”, Journal of Global Optimization, 1997, $11,341-359$.

111 David Amsallem, Julien Cortial, Kevin Carlberg and Charbel Farhat, "A method for interpolating on manifolds structural dynamics reduced-order models", International Journal for numerical methods in engineering, 2009.

112 Glover, F. W., \& Kochenberger, G. A. (Eds.). Handbook of metaheuristics (Vol. 57). Springer Science \& Business Media, 2006. 